Final Report

\title{
HIGH BETA AND \\ SECOND STABILITY REGION \\ TRANSPORT AND STABILITY ANALYSIS
}

M. H. Hughes, M. W. Phillips, A. M. M. Todd, J. Krishnaswami and R. Hartley

\author{
Grumman Corporate Research Center \\ 4 Independence Way \\ Princeton, N.J. 08540
}

September 1992

\author{
prepared for \\ Department of Energy \\ Office of Energy Research \\ Acquisition Management Division, ER-64 \\ Washington, D. C. 20545
}

Work performed under the auspices of the U.S. Department of Energy by Grumman Corporation under contract number

DOE Grant No. DE FG02-89ER51124

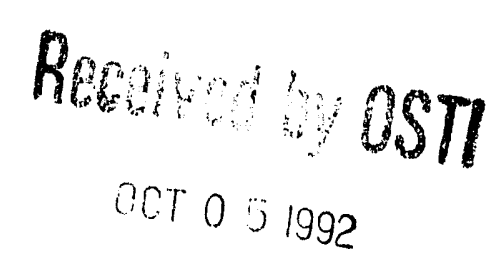

This document was prepared as an account of work sponsored by an agency of the United States Government. Neither the United States Government nor Grumman Corporation nor any of their employees makes any warranty, express or implied or assumes any legal liability for the accuracy, completeness or usefulness of any information. The views and opinions of authors expressed herein do not necessarily state or reflect those of the United States Government or Grumman Corporation. 


\begin{abstract}
This report describes ideal and resistive MHD studies of high-beta plasmas and of the second stability region. Emphasis is focused primarily on 'supershot' plasmas in TFTR where MHD instabilities are frequently observed and which spoil their confinement properties. Substantial results are described from the analysis of these high beta poloidal plasmas. During these studies, initial pressure and safety factor profiles were obtained from the TRANSP code, which is used extensively to analyze experimental data.

Resistive MHD stability studies of supershot equilibria show that finite pressure stabilization of tearing modes is very strong in these high $\beta_{p}$ plasmas. This has prompted a detailed re-examination of linear tearing mode theory in which we participated in collaboration with Columbia University and General Atomics. This finite pressure effect is shown to be highly sensitive to small scale details of the pressure profile. Even when an ad hoc method of removing this stabilizing mechanism is implemented, however, it is shown that there is only superficial agreement between resistive MHD stability computation and the experimental data. While the mode structures observed experimentally can be found computationally, there is no convincing correlation with the experimental observations when the computed results are compared with a large set of supershot data.
\end{abstract}

We also describe both the ideal and resistive stability properties of TFTR equilibria near the transition to the second region. It is shown that the highest $\beta$ plasmas, although stable to infinite- $n$ ideal ballooning modes, can be unstable to the so called 'infernal' modes associated with small shear. The sensitivity of these results to the assumed pressure and current density profiles is discussed.

Finally, we describe results from two collaborative studies with PPPL. The first involves exploratory studies of the role of the $1 / 1$ mode in tokamaks and, secondly, a study of sawtooth stabilization using ICRF. 
!

।

-

○

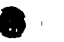

?

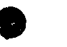

○ 


\section{CONTENTS}

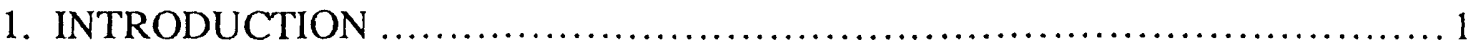

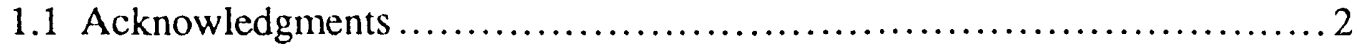

2. SUMMARY OF RESEARCH ACCOMPLISHMENTS ....................... 3

2.1 Studies of the Resistive Tearing Mode ................................ 3

$2.2 \mathrm{MHD}$ Analysis of High $\beta$ Supershot Plasmas........................... 5

2.3 Studies of Pressure Driven Modes......................................... 6

2.4 Studies of the $1 / 1$ Mode in Tokamaks.................................9

2.5 ICPF Sawtooth Stabilization ...................................... 9

3. MHD EQUILIBRIUM AND STABILITY CODES................................. 13

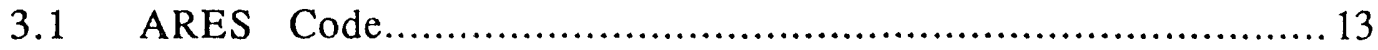

3.2 LOBAL Solver .................................................. 15

3.3 STBAL Solver .................................................... 16

3.4 Equilibrium Solver............................................. 16

3.5 Supershot Equilibria ........................................... 17

4. STUDIES OF THE RESISTIVE TEARING MODE .............................. 21

4.1 Finite Pressure Stabilization of the Tearing Mode ........................ 23

4.2 Sensitivity to the Assumed Pressure Profiles..................................25

4.3 Sensitivity to the Assumed Current Density Profile ...................... 26

4.4 Theoretical Studies of the Tearing Mode............................... 31

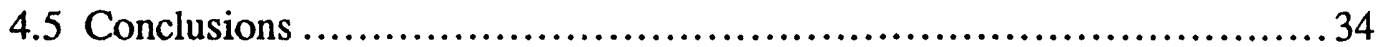

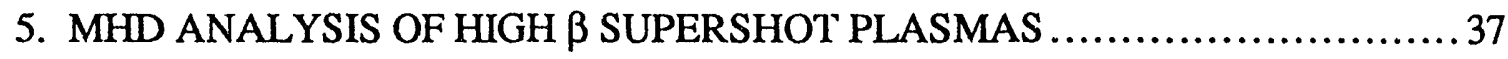

5.1 Analysis of Shot \#35782.............................................. 37

5.1 .1 Ideal Stability of Shot $\# 35782$................................ 39

5.1 .2 Resistive Stability ........................................ 42

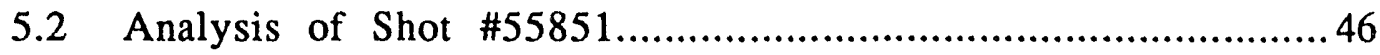

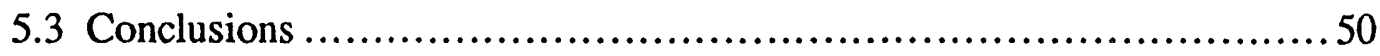

6. STUDIES OF PRESSURE DRIVEN MODES.....................................5 53

6.1 Sensitivity to the Pressure Profile......................................5 53

6.2 Sensitivity to the Current Density Profile ............................. 58

6.3 Conclusions ........................................................6 60

7. STUDIES OF THE 1/1 MODE IN TOKAMAKS.................................63

8. ICRF SAWTOOTH STABILIZATION ...................................... 71

8.3 Experimental ICRF Sawtooth Stabilization Observations...................... 72

8.4 Theoretical Stability Calculations ..................................... 74

8.5 Conclusions ...................................................... 76

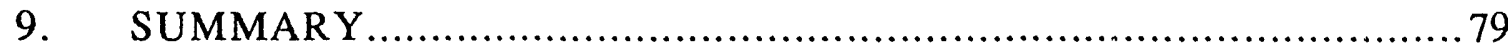

APPENDIX

Associated Publications and Presentations ..............................8 83 


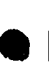<smiles>[Tl]</smiles> 


\section{INTRODUCTION}

This report describes the program of work carried out at the Grumman Corporate Research Center during the period 1990 through 1992 and supported by the Department of Energy under contract \#DE-FG02-89ER51124. This contract was awarded following our proposal submitted to the DoE in response to Special Research Grant Notice 89-4, Transport Initiative in Tokamak Fusion Plasmas. The original research plan submitted was divided into three tasks of which the following two were selected for support:

Task 1: MHD Stability Studies of Transport Simulations of the Second Region.

(a) Stability studies of transport simulations of second region access scenarios.

(b) Second stability region parameterization of beam heated plasmas.

(c) Resistive MHD analysis of high beta and high beta-poloidal plasmas.

Task 2: Time dependent Transport Modeling of Second Region Experiments.

(a) Investigate plasma profile modification for second region access.

(b) Study effects of RF heating on MHD stability.

All these tasks and topics which were specified in our original proposal, as well as new topics that have arisen subsequently, are addressed in this report. Specifically, Section 4 describes studies of the resistive tearing mode (Task 1(c)) while Sections 5 and 6 emphasize ideal MHD stability studies (Task 1(a) and (b), Task 2(a)). Section 7 considers some exploratory studies of the role of the $1 / 1$ mode in tokamaks while Section 8 describes ICRF sawtooth stabilization (Task 2(b)). The main conclusions of this study are summarized in Section 9.

Part of the work described in this report is already published in the literature. Further papers are currently in preparation and will be submitted for publication prior to the expiration of the present contract in December 1992. In addition, this work has been described at numerous conferences and workshops. The various publications and presentations are enumerated in the Appendix. 


\subsection{Acknowledgments}

The program of work described in this report has involved close interaction with other groups, in particular, those at the Princeton Plasma Physics Laboratory and Colurnbia University. Our principal contacts at these institutions were Dr. K. McGuire at PPPL and Professor G. Navratil at Columbia together with various members of their staff. In addition, we have maintained contact with the theory groups at both institutions. In particular, we established a close liaison with numerous members of the Theory Division at PPPL and with Professor A. Bhattacharjee at Columbia. Additionally, we have worked closely with Dr. John Greene of General Atomics on the subject of linear tearing mode theory. We have regularly contributed to, and benefited from, an informal MHD Study Group organized by Dr. D. Monticello at PPPL whose membership included both theorists and representation from the experimental groups.

We have enjoyed excellent working relationships with these groups and it is a pleasure to acknowledge their cooperation and many discussions which, we trust, were mutually beneficial. 


\section{SUMMARY OF RESEARCH ACCOMPLISHMENTS}

This Section gives a brief summary of the results obtained during the course of this study. A more detailed description of each topic is given in subsequent Sections of this report. The studies described here encompass each Task and each topic stated in the Introduction.

\subsection{Studies of the Resistive Tearing Mode}

Our library of MHD stability codes includes an extensively tested linear resistive code, known as ARES, developed previously under Grumman IR\&D funding. Here, ARES has been applied specifically to study the resistive MHD stability properties of low $\beta$ $(<0.4 \%)$, high safety factor, q, $(12)$ 'supershot' plasmas in TFTR where instabilities are often observed and there is a correlation between the appearance of a mode and subsequent deterioration of confinement. This is variously referred to as 'beta saturation' or 'beta collapse' depending on the severity of the confinement degradation. We have studied the possibility that the modes responsible for this behavior are purely resistive in character; specifically, 'tearing' modes[1]. This study is described in detail in Section 4 of this report.

Initial equilibrium pressure, $\mathrm{p}$, and $\mathrm{q}$ profiles were obtained directly from the TRANSP analysis code. The particular series of equilibria studied were first shown to be stable to ideal modes, at least with a wall-on-plasma boundary condition. The profiles were subsequently adjusted to find the conditions under which internal resistive modes can occur. In particular, it is found that elevated values of the safety factor on axis, $q(0)(>1)$, are required to excite resistive modes at all. This analysis shows, however, that these modes are readily stabilized in these high $\beta_{p}(\sim 2)$ supershot plasmas. While instabilities are found at relatively small values of the Lundquist number, $S$, these typically stabilize when $S$ is orders of magnitude smaller than the range of experimental interest. This is a manifestation, with interesting consequences, of the stabilizing influence of finite pressure anticipated theoretically by Coppi, Greene and Johnson [2] and subsequently analyzed by Glasser, Greene and Johnson (GGJ) [3].

A recent theoretical study by Bishop et al. [4] showed that the stability of the tearing mode is sensitive to details of the pressure profile in the vicinity' of the rational surface of interest. We arrived at the same conclusion independently, though computationally, by arbitrarily introducing narrow 'flat spots' in the pressure profile, centered at the rational surfaces. The stabilizing effect of the pressure is then reduced to a point where the tearing 
mode persists into the S-range of interest $\left(\sim 5 \times 10^{8}\right)$. Our studies suggest, however, that even with this artifice to remove the finite pressure stabilization there is only superficial agreement with the experimental data. Detailed comparison between the computational results and a large sample of experimental data does not show any convincing correlation.

An analytic study prompted by these computational results, in which we participated in collaboration with colleagues from Columbia University and General Atomics, has re-examined the predictions of linear tearing mode theory [5]. This exercise showed that there is a critical Lundquist number below which the pressure has no effect and, conversely, above which it is stabilizing. This critical value scales with the plasma parameters in exactly the same way that is predicted by GGJ. There is a coefficient, however, smaller than that in GGJ by an order of magnitude, which therefore supersedes the latter. It is shown in [5] that hydrodynamic mechanisms such as viscosity or mass flows cannot be ignored in this regime where the pressure is stabilizing. These can be either stabilizing or destabilizing.

The main conclusions of this study of the tearing mode can be summarized as follows:

- The finite pressure stabilization of the tearing mode is very strong in supershot plasmas where $\beta_{p}$ is large $(\sim 2)$.

- The stabilizing effect is ighly sensitive to small scale details of the pressure profile. The tearing mode persists only when we invoke some mechanism which flattens the pressure profile over narrow regions in the vicinity of rational surfaces.

- Even with flat spots in the pressure profile there is no convincing correlation between the computed results and the experimental data.

- Analytic calculations show that hydrodynamic effects omitted in linear tearing mode theory can be important in the regime where the pressure stabilizes the instability. 


\subsection{MHD Analysis of High $\beta$ Supershot Plasmas}

In these studies we sought to gain better understanding of the MHD activity seen during high beta supershots. In high beta TFTR supershot experiments, $m / n=3 / 2$ modes and occasionally $2 / 1$ modes are observed, accompanied by a degradation in confinement and a drop off in neutron yield. One candidate considered for these modes, and discussed elsewhere in this report, is a resistive mode which can occur when the pressure profile is flattened locally on a mode rational surface. Another candidate we considered is an infernal mode.

The supershots of interest were actually the best supershots recorded. Despite their record achievement, $90 \%$ of the shots have significant MHD activity. Some of the shots suffer a beta collapse soon after reaching peak beta. The reasons for considering an infernal mode for these shots was the observation that the modes get worse as beta gets higher. Typically only a single mode is ouserved. In addition, the pressure profile in supershots is extremely peaked. Previous ballooning mode analysis of these shots suggested the intriguing possibility that if $\mathrm{q}(0)>1.2$ the center of the plasma could be in the transition region between the 1 st and 2 nd regions of stability. Studies by us over the years have shown that when the central pressure profile is in the transition region infernal modes are a likely possibility.

We looked at several of the highest beta supershots including shots \#29918, \#35782 and \#55851. Profiles were obtained from TRANSP analysis of these shots. The main unknown in the analysis of these shots is the shape of central current profile or, alternatively, the central q profile. Indeed, in the TRANSP analysis of these shots the central q can vary as much as $20 \%$ depending on which features and specific parameter are used in the TRANSP run. A model was constructed that retains the shape of the current profile as supplied by TRANSP fixed, but can flatten the central portion of the profile thereby raising $q(0)$.

The main conclusions of this study of high $\beta$ supershots are:

- Supershots with low central shear are either very close to or in a regime unstable to infernal modes. In almost all cases some flattening of the central current profile, as given by TRANSP, is required. The flattening is modest e.g. $q(0) \sim 1.3$ is enough to see the $3 / 2$ mode. 
- The dominant mode depends on the trajectory the equilibrium follows in $\beta$, $q(0)$ space as time evolves. The $2 / 1$ mode requires $q(0)>1.5$ to occur which may explain why this mode is not often observed.

- Both infernal modes and ballooning mode instability regions in parameter space are surround by a layer unstable to resistive modes.

- These studies suggest that the MHD activity observed in supershots is associated with ballooning and/or infernal modes. Supershots can operate at or slightly in the ballooning mode threshold with an increase in MHD activity but no collapse. However, if the current profile in the center becomes flat, then a discrete infernal mode occurs leading to a beta collapse.

This study inspired a further exploration of the role that the pressure and current density profiles play in exciting infernal modes. This is summarized in the next Section.

\subsection{Studies of Pressure Driven Modes}

The analysis of high $\beta$ supershots introduced the possibility that pressure driven ideal modes, albeit modified by finite resistivity, could play an important role in these plasmas. These studies also used initial equilibrium profile data obtained in numerical form from the TRANSP code. The q-profile was systematically altered in the vicinity of the center using a technique described in Section 3.5. In Section 6, we pursue the study of these pressure driven instabilities further, using analytic approximations to the $\mathrm{p}$ and $\mathrm{q}$ profiles such that these can be more easily altered in a systematic fashion. Interestingly, it is shown that both $\mathrm{p}$ and $\mathrm{q}$, obtained initially from TRANSP, can be represented by a relatively simple analytic formulae. In this way, we have gauged the sensitivity of the results to the assumed profiles used to generate the equilibria.

Using an analytic form for the pressure together with the TRANSP q-profile, albeit modified as before to vary $q(0)$, we find the so called 'infernal' modes [6], which appear when $q(0)$ is close to a rational value (i.e. the shear within the rational surface of interest is small). These instabilities appear well below the onset of large toroidal mode number ballooning modes. It is shown that the limiting $\beta$ determined by the onset of the infernal mode follows a Troyon like scaling, $\beta_{\mathrm{N}}=\beta /(\mathrm{I} / \mathrm{aB})=\mathrm{C}_{\mathrm{T}}$, but that the value of $\mathrm{C}_{\mathrm{T}}$ depends sensitively on the peakedness of the pressure profile. In particular, the highly peaked 
pressure profiles that characterize supershot plasmas, while initially responsible for their good confinement, can ultimately impose a severe limit on the maximum attainable $\beta$.

The qualitative characteristics of the infernal modes are appealingly consistent with experimental observations. Thus, if $\mathrm{q}(0)$ increases during the beam heating phase, as suggested by the fact that the $1 / 1$ sawtooth mode vanishes shortly after the beams are switched on, then we would expect that the higher $\mathrm{m} / \mathrm{n}$ modes would be excited first, cascading to smaller n-values, as is often observed, as $q(0)$ continues to increase. Moreover, our computational results show that the $2 / 1$ mode is excited only when the pressure profile is very highly peaked. This, together with the requirement that $q(0)$ must also be highly elevated $(\sim 1.8)$ to excite the $2 / 1$ infernal instability, could be the reason that the $2 / 1$ mode is observed very infrequently ( $2 \%$ of all TFTR plasmas).

A similar exercise in which the pressure profile is fixed but the shape of the $q$ profile is altered such that the current density becomes more peaked demonstrates that, as the shear within the rational surface of interest is increased, the infernal mode stability limit increases up to the point where small toroidal mode number ballooning modes are excited. Subsequently, further peaking of the current profile reduces the maximum attainable $\beta$. In this ballooning mode regime, the larger n-numbers are excited first, in accordance with analytic theory, and do not rely on elevated values of $q(0)$. These ballooning instabilities can impose limiting $\beta$ values comparable with those determined by the infernal mode.

The main conclusions of this study of ideal pressure driven modes can be summarized as follows:

- Ideal MHD theory predicts that high $\beta$ supershot plasmas are unstable to either low- $n$ ballooning modes or infernal modes, depending on the peakedness of the current density profile. At low $\beta$, the comparison between theory and experiment is less convincing unless the pressure profiles in those plasmas observed to be unstable are substantially more peaked than is predicted by TRANSP.

- Infernal modes are excited only when the shear is small and $q(0)$, therefore, is close to a rational value. Bursts of instability appear in narrow bands at elevated $q(0)$ values. 
- $\quad$ The limiting $\beta$ for stability to the infernal mode follows a Troyon like scaling, $\beta=C_{T}(\mathrm{I} / \mathrm{aB})$, but the value of $\mathrm{C}_{\mathrm{T}}$ depends sensitively on the peakedness of the pressure profile. These instabilities can severely limit the maximum attainable $\beta$ especially when the pressure profiles are highly peaked.

- The $3 / 2$ infernal mode is the most unstable and, for reasons not yet understood, the $2 / 1$ instability is excited only when the pressure profile is very highly peaked. This, together with the highly elevated values of $q(0)$ (>1.7) required to excite the mode, could be a reason for the relatively infrequent appearance of the $2 / 1$ instability in the experiments (about $2 \%$ of all plasmas).

- If the shear is substantially larger than is predicted by TRANSP, the unstable modes are ordinary low- $n$ ballooning modes. These instabilities do not rely on elevated valves of $q(0)$, and a mode with a given toroiclal mode number can be excited over a wide range including $q(0)=1$. In this case the higher $\mathrm{n}$ numbers are always the most unstable, in accordance with analytic theory. 


\subsection{Studies of the $1 / 1$ Mode in Tokamaks}

The sawtooth instability in ohmically heated plasmas is a phenomenon known since the earliest days of tokamak research. This topic still attracts considerable attention but is still poorly understood, however. Thus, in collaboration with W. Stodiek of PPPL, we have initiated some exploratory studies of the role of ideal and resistive $1 / 1$ modes in ohmically heated plasmas.

Equilibrium profile data were obtained from the TEXTOR tokamak and these were analyzed using the ARES codes. We concentrated on two cases where: (a) qedge $>3$ and the current density profiles, suitably normalized, develop into an universal shape independent of the major and minor radii, the toroidal field and the total current; (b) 'extreme' cases where qedge 2 and the measured current density profile shows a distinct plateau within the $\mathrm{q}=1$ radius. In each case the central value of the safety factor was substantially less than unity and the shear at the $q=1$ surface was controlled by varying the width of the current plateau within the rational surface.

It is well known that the ideal internal $1 / 1$ kink mode can be stable in toroidal geometry even when $\mathrm{q}<1$. We find that both the extreme and universal experimental profiles mentioned above lie close to marginal stability for this mode. In addition, when using the version of ARES which imposes the constraint of incompressible perturbations, we found conditions which were also stable to resistive modes, when the shear at the $q=1$ surface was sufficiently reduced. When a version of ARES including fluid compressibility became available, however, this regime of complete MHD stability vanished. A resistive MHD mode was found at low shear which becomes stable in the limit of incompressibility.

To recapitulate, in the more realistic situation with finite fluid compressibility, we are unable to find conditions stable to resistive $1 / 1$ modes, which contradicts the experimental observations. Our preliminary conclusion is that either the requirement of zero growth rates is too stringent, or that the 'usual' resistive MHD model is inadequate.

\subsection{ICRF Sawtooth Stabilization}

A collaborative effort with the RF group at PPPL was undertaken to understand ICRF sawtooth stabilization on TFTR. The role of the Grumman group in this study was to evaluate the MHD stability of ICRF stabilized plasmas using data from the TRANSP 
analysis of these shots. These results were used in a theoretical model based on energetic ion stabilization of $m=1 \mathrm{kink}$ instabilities to evaluate the stability of the plasmas. The principal investigator, and our contact, for this work was C. K. Phillips (PPPL) who was responsible for integrating the MHD calculations with the energetic ion stabilization model and for comparisons with experimental data.

Stabilization of sawtooth oscillations using ICRF heating has been observed on several tokamaks including TFTR and JET. In TFTR the stabilization of sawteeth has been observed both in RF only as well as in combined RF and NBI heated plasmas. The ICRF sawtooth stabilized phase in TFTR experiments can last up to many times the energy confinement time. The stabilized phase typically terminates with a very rapid growth of the $\mathrm{m}=1$ island, leading into a large sawtooth crash. Theory suggests that the fast ions produced by the ICRF heating suppress sawteeth by stabilizing the $m=1$ instabilities which are believed to be responsible for the sawtooth oscillations.

Stabilization of sawtooth oscillations using ICRF is potentially important at high plasma currents when NBI heating alone is insufficient to stabilize sawtooth oscillations. It may be of particular importance during D-T operations on TFTR. It is hoped that using ICRF heating will help drive TFTR plasmas towards the $Q=1$ break-even poirt during $D$ $T$ operations. The accompanying increase in the energy confinement time and strong peaking of the core plasma temperatures may lead to an enhancement of the fusion reactivity or $\beta_{\alpha}$.

For these studies the experimental parameters were obtained directly from measurements as well as from TRANSP and SNAP analyses. The ideal MHD stability of ICRF stabilized plasmas was evaluated using the ARES code. These results were used in the evaluation of a theoretical model that includes stabilization of energetic ions. The results were compared with the experiments. Throughout the sawtooth stabilization phase, the discharge evolves towards the region of phase space characterized by $m=1$ modes. Comparison of the experimental stabilized discharge parameters to the $\mathrm{m}=1$ ideal internal kink stability boundaries qualitatively supports the interpretation that the stabilization is due to the pressure of the hot ions. 
The main conclusions found thus far in this study are:

- A higher threshold power for stabilization at the lower plasma current is observed. This is consistent with theoretical models that suggest the stabilizing influence of energetic trapped hot ions decreases if the hot ion pressure profile is not peaked and well-contained within the $q=1$ volume.

- A qualitative correlation has been found between the experimental observations of ICRF sawtooth stabilization and the theoretical predictions, given the large uncertainties in both the measurements and the theory.

[1] Furth, H.P., Killeen, J. and Rosenbluth, M.N., Phys Fluids, 6, (1963), 459.

[2] Coppi, B,. Greene, J.M., and Johnson, J.L., Nuclear Fusion, 6, (1966), 101

[3] Glasser, A.H., Greene, J.M. and Johnson , J.L., Phys. Fluids, 18, (1975), 875

[4] Bishop, C.M., Hastie, R.J., Connor, J.W. and Cowley, S.C., Plasma Phys. and Controlled Fusion, 33, (1991), 389.

[5] Iacono, R., Bhattacharjee, A., Ronchi, C., Greene, J.M., and Hughes, M.H., in preparation, to be submitted for publication in Phys. Fluids B

[6] J. Manickam, N. Pomphrey, A. M. M. Todd, Nucl. Fusion 27, (1987), 1461. 


\section{MHD EQUILIBRIUM AND STABILITY CODES}

The studies described in this report have exploited a number of computer codes whose main features are conveniently summarized below. Each of the MHD stability codes described here (Sections 3.1 - 3.3) were developed previously at Grumman. The equilibrium solver described in Section 3.4 is a variant of the flux coordinate solver originally developed at PPPL [1] but modified extensively by ourselves. It is also convenient, at this point, to summarize the procedure that we have adopted for calculating supershot equilibria; this appears in Section 3.5.

\subsection{ARES Code}

Our library of codes for MHD stability analysis includes a linear resistive code, known as ARES [2]. Apart from linearization, the only further assumptions implemented in ARES are that the perturbations are incompressible and that the plasma is in contact with a conducting wall. The assumption of incompressibility is valid in resistive MHD where growth times are typically long compared with the transit time of sound waves. It then proves convenient to introduce a ma tic vector potential, $\overrightarrow{\mathbf{a}}$, and a vector stream function, $\overrightarrow{\mathbf{u}}$, such that the magnetic field, $\overrightarrow{\mathbf{b}}=\vec{\nabla} \times \overrightarrow{\mathbf{a}}$, and the velocity $\overrightarrow{\mathbf{v}}=\vec{\nabla} \times \overrightarrow{\mathbf{u}}$. The MHD equations then take the form :

$$
\begin{aligned}
& \frac{\partial \overrightarrow{\mathbf{a}}}{\partial \mathrm{t}}=(\vec{\nabla} \times \overrightarrow{\mathbf{u}}) \times \overrightarrow{\mathbf{B}}-\frac{1}{\mathrm{~S}} \vec{\nabla} \times \vec{\nabla} \times \overrightarrow{\mathbf{a}}+\vec{\nabla} \zeta \\
& \frac{\partial}{\partial \mathrm{t}}(\vec{\nabla} \times \vec{\nabla} \times \overrightarrow{\mathbf{u}})=\vec{\nabla} \times(\overrightarrow{\mathbf{B}} \cdot \vec{\nabla}(\vec{\nabla} \times \overrightarrow{\mathbf{a}})+(\vec{\nabla} \times \overrightarrow{\mathbf{a}}) \cdot \vec{\nabla} \overrightarrow{\mathbf{B}})
\end{aligned}
$$

where we use the convention that lower case letters represent perturbed quantities while capitals refer to equilibrium values. To define $\overrightarrow{\mathbf{a}}$ and $\overrightarrow{\mathbf{u}}$ uniquely we adopt the gauge such that $\nabla \zeta=0$ and require that the toroidal component of $\overrightarrow{\mathbf{u}}$ should vanish. These equations are then written in a non-orthogonal magnetic flux coordinate sysiem, Fourier analyzed to describe their poloidal and toroidal dependencies, and the resulting evolution equations for the harmonic amplitudes are solved numerically as an initial value problem using finite differences in the 'radial' direction only. The convolution sums which appear are evaluated by a Fast Fourier Transform method. The radial differencing implements a staggered mesh scheme which ensures that vector identities such as $\vec{\nabla} \cdot \vec{\nabla} \times \overrightarrow{\mathbf{a}}=0$ are satisfied exactly by the numerical differential operators. This is a crucial requirement in MHD stability codes. 
The staggered mesh also permits the boundary conditions to be applied in a consistent manner. For the conducting wall situation used here these are simply that the perpendicular components of $\overrightarrow{\mathbf{b}}$ and $\overrightarrow{\mathbf{v}}$ must vanish at the boundary. The remaining components are then calculated consistently without invoking any further ad-hoc physics. In addition, since the low frequency, long wavelength MHD phenomena of interest evolve on timescales that are long compared with the fastest normal modes of the system we use a fully implicit temporal differencing scheme which both eliminates fast modes and avoids severe timestep restrictions when the resistive term is locally large. This procedure leads to an efficient code which enables large numbers of calculations to be carried out quickly. This is particularly important since each calculation must be converged both in the number of Fourier harmonics used and in the number of radial surfaces employed. The code allows for up to 500 surfaces and 20 poloidal harmonics. A typical calculation runs in a few minutes on the NERSC Cray II.

For completeness, we have also constructed a second version of the code, ARESII [3], in which the constraint of incompressibility is relaxed. This solves the full set of linear equations:

$$
\begin{aligned}
& \frac{\partial \overrightarrow{\mathbf{a}}}{\partial t}=\overrightarrow{\mathbf{v}} \times \overrightarrow{\mathbf{B}}-\eta \vec{\nabla} \times \vec{\nabla} \times \overrightarrow{\mathbf{a}}+\vec{\nabla} \xi \\
& \rho \frac{\partial \overrightarrow{\mathbf{v}}}{\partial t}=-\vec{\nabla} \mathrm{p}+(\overrightarrow{\mathbf{B}} \cdot \vec{\nabla}) \overrightarrow{\mathbf{b}}+(\overrightarrow{\mathbf{b}} \cdot \vec{\nabla}) \overrightarrow{\mathbf{B}}-\vec{\nabla}(\overrightarrow{\mathbf{B}} \cdot \overrightarrow{\mathbf{b}}) \\
& \frac{\partial \mathrm{p}}{\partial t}+(\overrightarrow{\mathbf{v}} \cdot \vec{\nabla}) \mathrm{P}=-\Gamma \mathrm{P} \vec{\nabla} \cdot \overrightarrow{\mathbf{v}}
\end{aligned}
$$

where $\Gamma$ is the adiabatic index. While we have retained the vector potential description of the magnetic perturbation, it proves convenient to replace the vorticity equation used previously by the momentum equation. This more primitive formulation avoids the difficulties described in [4] where the vorticity equation is retained together with an additional equation for $\nabla \cdot \overrightarrow{\mathbf{v}}$. That approach leads to third derivatives with respect to the radial coordinate which complicate the problem unnecessarily and result in a larger set of equations to be solved. The numerical treatment of the above equations, including compressibility, is identical with that implemented in the original version of ARES. 


\subsection{LOBAL Solver}

The LOBAL code was designed specifically to calculate the beta limit of low-n MHD ballooning modes and infernal modes. The chief strength of LOBAL is its' speed. Runs typically take only ten to twenty seconds on a workstation. A normalization is used that keeps the modes discrete as they go through the marginally stable point from instability to stability. This makes extrapolation of the marginally stable point easier and more accurate. Standard MHD codes could also be used to find beta limits however, they tend to be slow (i.e. minutes per run). In addition, it is often difficult to extrapolate the marginally stable point from the MHD growth rate because of numerical problems as the growth rate goes to zero. This is because the frequency of a given mode changes abruptly at the marginal stability point. This change is a result of the mode changing in character from a discrete unstable mode to a stable mode that is part of the continuum.

The formalism used in LOBAL is due to Binaeu [5]. By choosing a different normalization, the three components of the MHD equation can be reduced to a single equation for just one component. The resulting equation is

$$
-\omega^{2} \frac{\mathbf{X}}{|\nabla \psi|^{2}}=\mathbf{B} \cdot \nabla\left(\frac{\nabla \psi \cdot \nabla \mathbf{Z}}{|\nabla \psi|^{2}}\right)+\frac{\mathbf{J} \times \nabla \psi \cdot \mathrm{Z}}{|\nabla \psi|^{2}}+\mathbf{B} \cdot \nabla\left(\frac{\mathbf{B} \cdot \nabla \mathbf{X}}{|\nabla \psi|^{2}}\right)+2 \kappa \mathbf{X}
$$

where $\nabla \cdot \nabla_{\|} Z=\nabla \cdot\left[\frac{\nabla \psi}{|\nabla \psi|^{2}} \mathbf{B} \cdot \nabla \mathbf{X}-\frac{\mathbf{J} \times \nabla \psi}{|\nabla \psi|^{2}} \mathbf{X}\right]$

The second equation can be formally inverted for $\mathrm{Z}$ and substituted into the first equation to give a single equation. This is in fact what is done numerically. Thus, the matrix resulting from the discretized equations is considerably reduced compared to the standard MHD equations. The quantity $\kappa$ is a type of curvature

$$
\boldsymbol{\kappa}=\frac{\mathbf{J} \times \mathbf{n} \cdot \nabla(\mathbf{B} \cdot \mathbf{n})}{|\nabla \psi|^{2}}, \text { where } \mathbf{n}=\frac{\nabla \psi}{|\nabla \psi|}
$$

The Binaeu formalism was extended to an energy principle $t$, R. C. Grimm et al. [6]. The PEST II code was developed using the energy principle formalism. Unlike the PEST II code, LOBAL solves the partial differential equation directly rather than using the energy principle approach. There is no particular advantage to this approach for basic MHD. If we wished to extend the code at some future date to include some kinetics effects, it is more 
straight forward to deal with the partial differential equation directly since the energy integral loses its meaning.

The differential equation is Fourier decomposed in the axisymmetric and azimuthal directions. The remaining radial direction is finite differenced in a fashion that keeps the matrix consisting of discretized equations symmetric. The eigenvalues are isolated using bisection then refined by shifting and inverse iteration.

\subsection{STBAL Solver}

The STBAL code is a high-n ideal MHD ballooning mode code. In some respects it is similar to other ballooning mode codes in that it can compute the stability of a give flux surface and compute the critical n number for instability. A chief difference is that it includes the perturbed equilibrium effects in the ballooning equation [7]. These effects allow the 1 st and 2 nd region critical pressure gradients to be computed. Whether a given equilibrium is in the 1 st region, the 2 nd region or in between, can then be ascertained. In addition, a local $S$ - $\alpha$ diagram can be generated giving a full picture of where a given field line lies with respect to the second region of stability. The predictions of 1 st and 2 nd region critical pressure gradients tend to be fairly accurate, thus allowing optimum profiles to be generated. An interface for optimizing profiles together with the equilibrium code has been written into the code. The prediction of the optimum profiles is such that typically only a few iterations are needed to generate a fully self consistent optimized ballooningequilibrium computation.

\subsection{Equilibrium Solver}

The equilibrium solver is the basis for all equilibrium and stability calculations. Accurately calculated equilibria are essential before reliable stability computations can be performed. Over the years we have found an increasing need for much more accurate numerically calculated equilibria. Careful convergence studies of unstable modes have shown that standard sized grids and tolerances which were previously thought to be adequate, were actually insufficient to numerically converge many types of modes. This is particularly true when calculating resistive modes which can have a small growth rate and whose eigenfunctions can have sharp gradients. Often, several hundred radial surfaces are required to compute equilibria with sufficient accuracy to observe the proper numerical convergence in the stability codes. 
The code we use is known as EQGRUM. It is a descendant of the EQ002 code originally written by Mike Reusch and a number of other authors at PPPL. The code solves toroidally symmetric ideal MHD equilibrium in a flux surface coordinate system [8]. The EQGRUM code still bears a superficial resemblance to the original EQ002 code but has been extensively modified. A key feature is an internal flux averaged equilibrium solver. This is used for q profile specification (unlike EQ002 which uses a different method), J•B profiles, and for converging to a specified toroidal beta. Many features have been fixed and added to the code including a new iteration method, a more accurate method of calculating the center point, and the ability to restart from different sized grids. Interfaces have been added to directly read in data from transport codes such as TRANSP and BALDUR. In addition, numerous new pressure, $\mathrm{q}$ and $\mathrm{J} \cdot \mathrm{B}$ profiles have been added as more refined models of experimentally observed profiles have evolved. The code has also been standardized to run on UNIX systems.

\subsection{Supershot Equilibria}

Toroidal equilibria are calculated using the 'fixed boundary', flux coordinate solver [1] described in Section 3.4. Thus, with the shape of the outermost flux surface prescribed, this code simply requires, for example, the pressure profile, $p(\Psi)$, and the safety factor, $q(\Psi)$, as input. Initial profiles are obtained in numerical form from the transport analysis code, TRANSP $[9,10]$ which employs all the experimental data that are available and attempts to calculate those parameters that are not measured. Thus the qprofile is obtained by solving the induction equation with models for the resistivity, noninductive current drive, sawteeth etc. and is therefore still subject to uncertainty. Similarly, the neutral beams, which serve to fuel supershot plasmas, can contribute a substantial fraction (30-50\%) of the total pressure and this is calculated using a Monte Carlo method with inevitable large statistical errors near the magnetic axis. The neutral beam deposition profile also impacts the calculated q-profile. We therefore allow ourselves some freedom to adjust these profiles as necessary. In particular, we shall need to modify the current density profile in a systematic fashion. This procedure, used extensively throughout the present study, is described below.

To map out the parameter range of the instabilities we adjust the pressure and $q$ profiles around the original data. This can identify the modes that occur near the equilibrium profiles and perhaps even suggest what the actual experimental profiles really are. To vary $\beta$, we simply scale the central pressure keeping the shape of the pressure profile constant. To change $q(0)$, we first use the equilibrium solver to generate the 
corresponding distribution of the surface function $\langle J \cdot B\rangle \mid\langle B \cdot \nabla \phi\rangle$. We then retain the shape of the $\langle J \cdot B\rangle \mid\langle B \cdot \nabla \phi\rangle$ profile but map $\psi$ to expand the profile in the vicinity of the axis while keeping the total current constant. The mapping function used for this purpose is

$$
\bar{\psi}_{\text {new }}=\bar{\psi}^{[\alpha+(1-\alpha) \bar{\psi}]}
$$

This procedure has the effect of changing the current density profile primarily in the center where the data is most uncertain. For example, Fig. 3.1(a) shows the initial $\langle\mathrm{J} \cdot \mathrm{B}\rangle \mid\langle\mathrm{B} \cdot \nabla$ $\phi>$ distribution generated using TRANSP data from shot 35782 taken at $t=4.0$ seconds, together with modified profiles obtained using the above procedure. Figure 3.1(b) shows the corresponding q profiles. For this particular TRANSP calculation the sawtooth model was not included resulting in initial values of q less than unity near the center. Subsequent TRANSP runs, which included a model for sawtooth induced transport, predict q-values everywhere greater than unity, highlighting the large uncertainties in the q-profile.

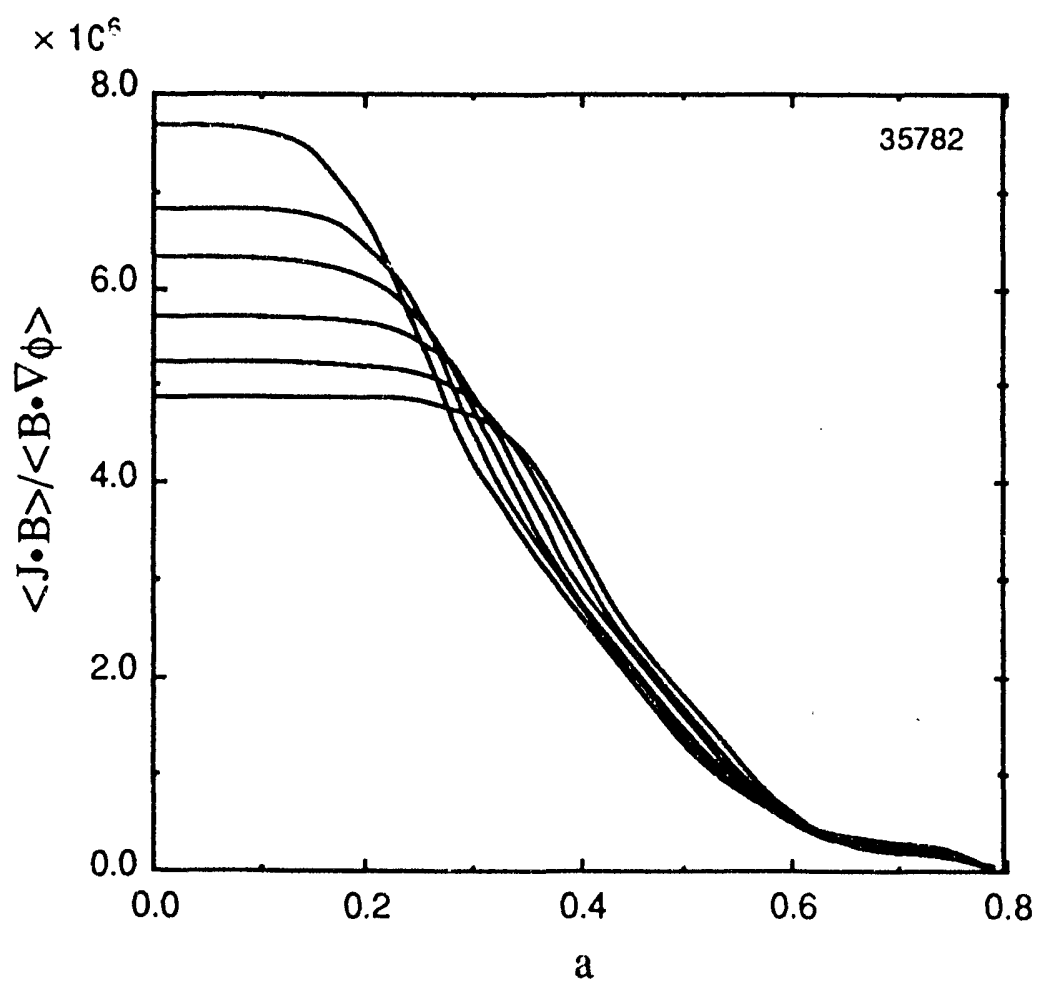

Fig. 3.1(a). Plot of $\langle J \cdot B\rangle \mid\langle B \cdot \nabla \phi\rangle$ (units $A / m$ ) vs minor radius showing maximum range of current profile variation used in these studies. 


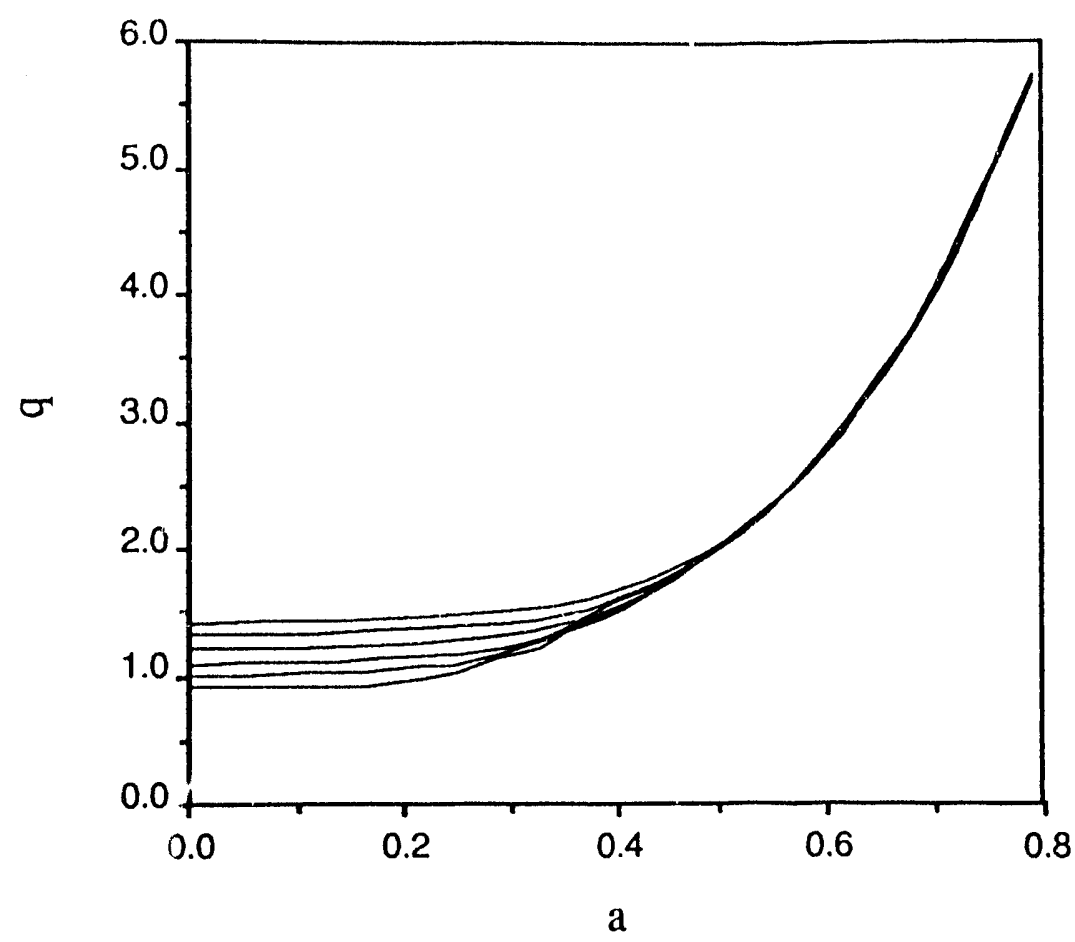

Fig. 3.1(b). Plot of $q$ vs minor radius for the $\langle\mathrm{J} \cdot \mathrm{B}\rangle \mid\langle\mathrm{B} \cdot \nabla \phi\rangle$ given in Fig. 3.1(a).

[1] DeLucia, J., Jardin, S.C. and Todd, A.M.M, J. Comp. Phys. 37, (1980) 204.

[2] Hughes, M.H., Phillips, M.W. and Storer, R.G. accepted for publication in Computer Physics Communications.

[3] Hughes, M.H., to be submitted to Computer Physics Communications.

[4] Charlton, L.A., Holmes, J.A., Lynch, V.E., Carreras, B.A. and Hender. T.C., J.Comp.Phys., 86, (1990), 270

[5] Bineau, M., Nucl. Fusion 2 (1962), 130.

[6] Grimm,R.C., Dewar,R.L. and Manickam J., J. Comp. Phys. 49, (1983), 94-117

[7] Phillips,M.W., Hughes,M.H., Todd,A.M.M., Okabayashi,M., Kaye,S.M., LeBlanc, B., Phys. Fluids B 2(5), May 1990

[8] DeLucia, J., Jardin, S.C. and Todd,A.M.M., PPPL-1564

[9] Hawryluk, R., in Physics of Plasmas Close to Thermonuclear Condidtions (Commisssion of the European Communities, Brussels, 1979), Vol. 1, p. 19.

[10] Gcldston,R.J., McCune, D.C., Towner H.H., Davis S.L., Hawryluk R.J. and Schmidt, G. L., J. Comput. Phys. 4361 (1981). 
0

।

0

।

.

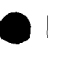

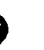

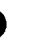

. 


\section{STUDIES OF THE RESISTIVE TEARING MODE}

MHD activity is often observed in TFTR supershot plasmas. Moreover, there is a correlation between the appearance of MHD instability in these plasmas and subsequent deterioration of their confinement. This phenomenon is variously referred to as 'betasaturation' or 'beta-collapse' depending on the severity of the confinement degradation. A mode with toroidal mode number $\mathrm{n}=2$ is excited most frequently, though modes with $\mathrm{n}=$ 1,3 and 4 are also observed. When $\beta_{\mathrm{N}}=\beta /(\mathrm{I} / \mathrm{aB})$ is small $(<2)$ there is MHD activity in approximately $50 \%$ of all discharges. On the other hand, at larger values of $\beta_{\mathrm{N}} \sim 2.7$ some $90 \%$ of the shots exhibit instability. To elucidate the nature of the instabilities responsible for this behavior we have undertaken a detailed study of the predictions of both ideal and resistive $\mathrm{MHD}$ theory based on the experimental data. In this Section we shall study the predictions of the single fluid, resistive MHD model. The further possibility of ideal (infinite conductivity) modes in supershot plasmas is deferred to Sections 5 and 6.

In view of the relatively small mode growth rates that are observed experimentally in supershots, it has been supposed that the instabilities of concern could conceivably be purely resistive in character; specifically, 'tearing modes' [1]. A feature long regarded as a curiosity, however, is the frequent appearance of a $3 / 2$ instability in these plasmas when $\Delta^{\prime}$ analysis of particular shots performed at TFTR (using a code employing cylindrical symmetry) and more sophisticated toroidal calculations described in the literature [2], suggest that tearing modes with this structure should be stable $\left(\Delta^{\prime}<0\right)$. We have reexaminer. these same supershot data in more detail using the ARES code and exploiting the techniques described in Section 3.5 to make small adjustments to the equilibrium parameters. Initially, then, we concentrated our attention on the possibility that the observed instabilities are indeed resistive tearing modes despite the conclusions from the $\Delta^{\prime}$ calculations mentioned above.

Specifically, we have studied the 266-series of high $\mathrm{q}(\sim 12)$, low $\beta(\sim 0.4 \%)$ plasmas whose evolution is shown in Fig. 4.1. Referring to Fig. 4.1, we note that these three diagrams summarize the evolutivi of some 50 individual shots which were analyzed extensively using TRANSP and subsequently grouped according to their MHD behavior. Thus, the initial evolution of each series, up to the onset of instability, is remarkably similar. The interesting question, then, is why these plasmas subsequently exhibit quite different MHD characteristics when their 'initial conditions' are nominally 

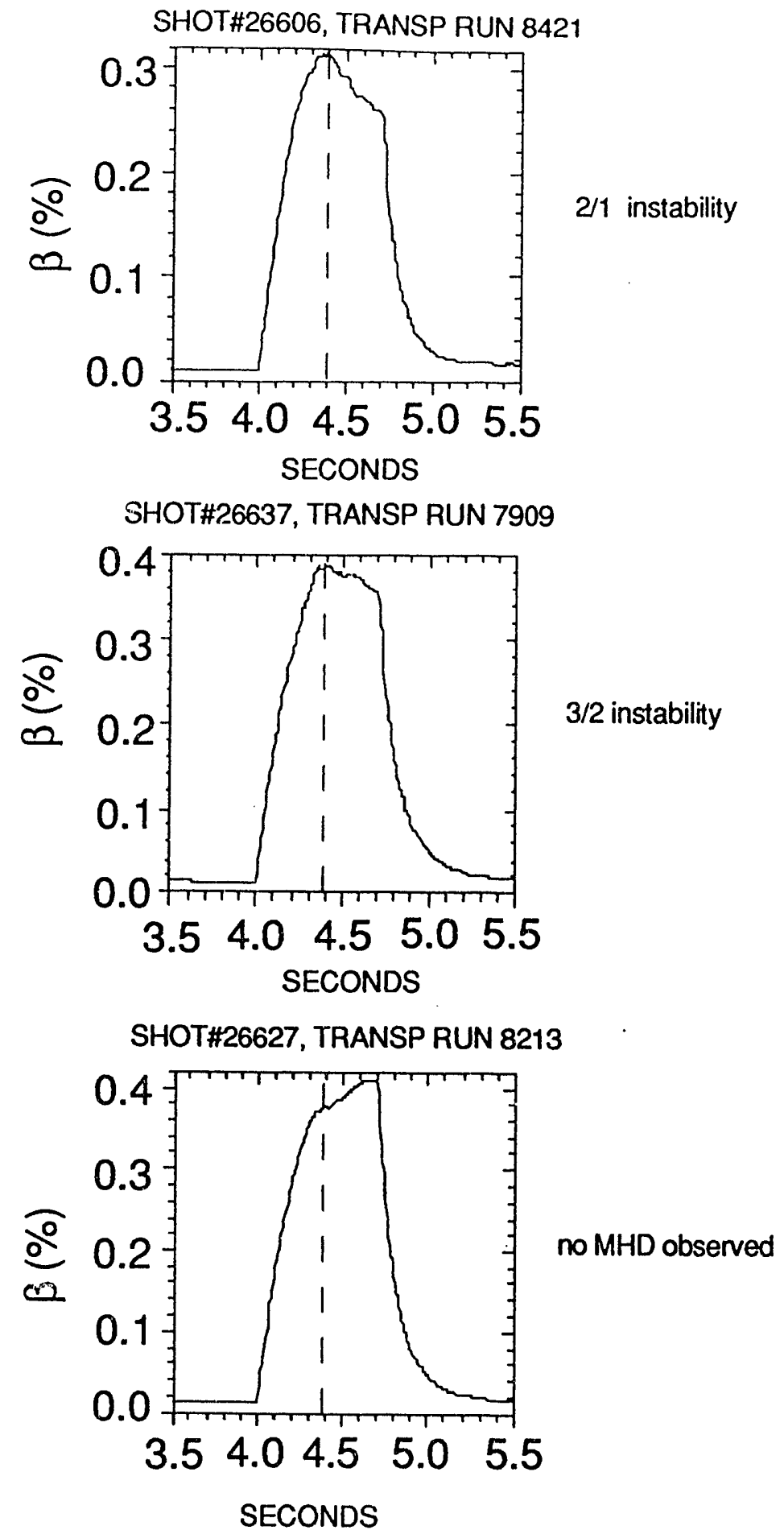

Fig. 4.1 Evolution of $\beta$ in the 266 -series of supershot plasmas.

The initial development of each case is nominally identical. Their MHD characteristics, however, are quite different. The dashed line indicates the time at which data was selected for analysis. 
identical. The statistics of the individual discharges compr: sing this particclar series are significant. Thus, in one single discharge a $2 / 1$ instability was observed while of the remainder approximately half were stable and the other half were unstable to a $3 / 2$ mode. In this case we search to find equilibrium conditions that are close to $3 / 2$ instability, or marginally stable to this mode, with the (unusual) possibility of exciting a $2 / 1$ mode.

\subsection{Finite Pressure Stabilization of the Tearing Mode}

Pressure and current density profiles obtained directly from TRANSP just before the observed onset of instability were used to reconstruct the equilibria for the supershots labeled 26606, 26637 and 26627. Subsequent MHD analysis of these equilibria shows that they are stable to ideal modes, at least with the 'wall-on-plasma' boundary condition used here. Using ARES, the initial equilibrium data obtained from TRANSP, where $q(0) \sim 1$, were also found to be resistively stable (by which we mean that no growing modes could be found) and in agreement with the $\Delta^{\prime}$ calculations mentioned above. However, when the current density profile is adjusted such that $q(0)$ is increased, or more importantly that the shear at the rational surfaces is correspondingly decreased, we find that a resistive mode eventually appears when $\mathrm{q}(0)$ exceeds some minimum value, at least when the Lundquist number, $S$, is small. This is illustrated in Fig. 4.2 which shows that, when $E S$ $=5 \times 10^{5}$, for example, there is a $3 / 2$ mode which first appears when $q(0)>1.18$ and, further, a $2 / 1$ instability which is excited when $q(0)>1.3$. Fig. 4.3 shows the $2 / 1$ mode growth rates as functions of the Lundquist number with $q(0)$ kept constant at a value exceeding the onset for instability. The $3 / 2$ instability exhibits similar behavior. We find that as the Lundquist number is increased the mode growth rates decrease very rapidly and, typically, the modes tend to stabilize at values of $S$ orders of magnitude smaller than those of experimental interest (viz. $\varepsilon S \sim 2 \times 10^{8}$, where $\varepsilon$ is the inverse aspect ratio). This is illustrated in Fig. 4.3 where the $n=1$ mode is stable when $\varepsilon S>3 \times 10^{6}$ This is a manifestation, with practical significance, of the finite pressure stabilization of tearing modes originally anticipated theoretically by Coppi, Greene and Johnson [3] and analyzed in detail by Glasser, Greene and Johnson (GGJ) [4]. A subsequent theoretical discussion by Hastie et al. [5] using simple model equilibria to evaluate the coefficients of GGJ, showed that a useful figure of merit for predicting the stability of the tearing mode is the product $\Lambda=\beta_{\mathrm{p}}^{5 / 6} \varepsilon^{2} S^{1 / 3}$. Thus, when $\Lambda$ is sufficiently large, the tearing modes are stable for any $\mathrm{m} / \mathrm{n}(\sim 50$ in Hastie's examples) and this shows that for a given aspect ratio 


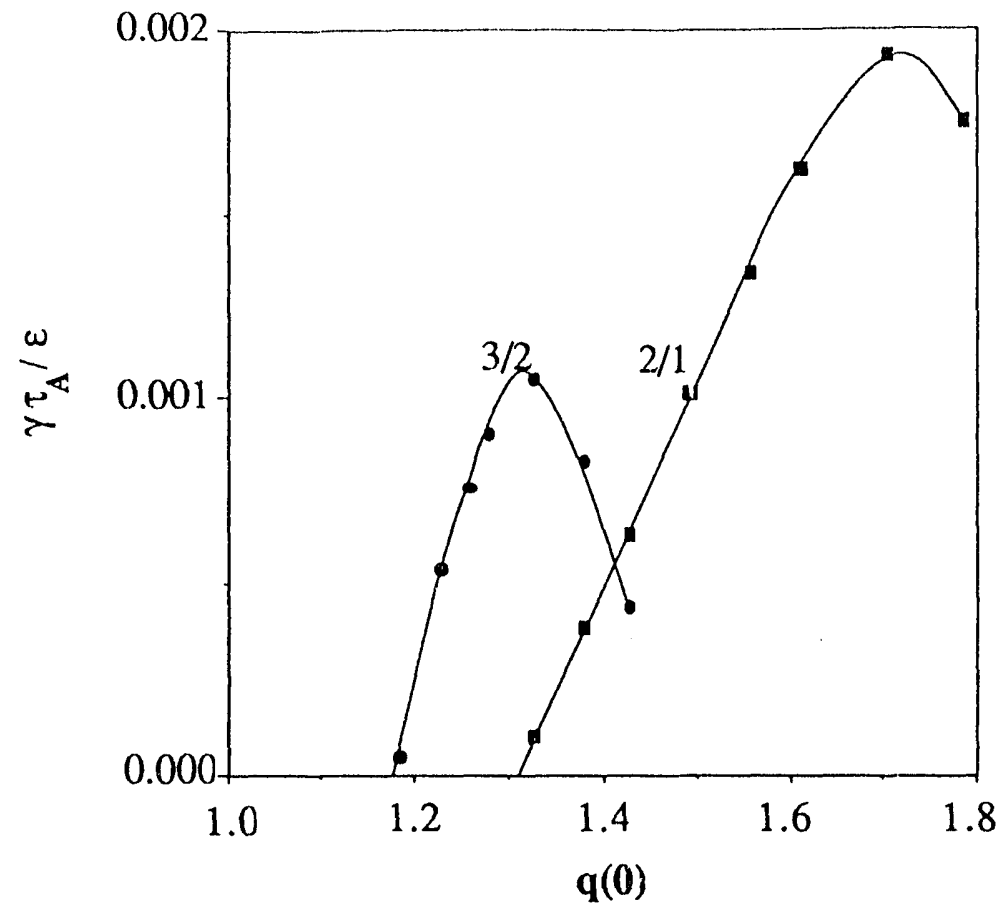

Fig. 4.2 Resistive mode growth rates as $\mathrm{q}(0)$ is varied.

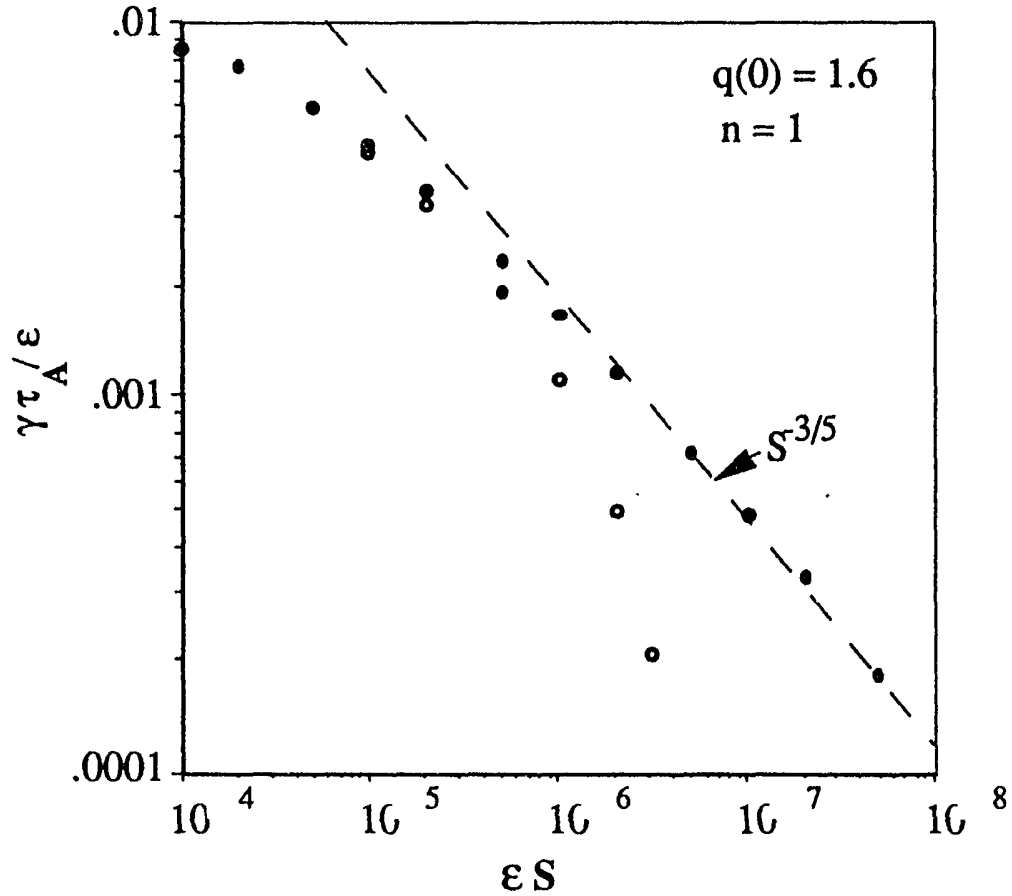

Fig. 4.3 Variation of resistive mode growth rate with the Lundquist number. The open symbols illustrate the stabilizing influence of the pressure on the tearing mode. The solid symbols show how the mode persists when the pressure is flattened over a narrow region centered at the rational surface. 
and given $S$ the mode stabilizes when $\beta_{\mathrm{p}}$ is correspondingly large. In supershot plasmas $\beta_{p} \sim 2$, typically resulting in values of $\Lambda(>10)$ such that strong stabilization would then be expected and, as shown in Fig. 4.3 is, indeed, found computationally.

We have, ourselves, recently participated in a detailed study of this finite pressure stabilization effect in collaboration with theorists from Columbia University [6]. This work also predicts that the tearing mode is stabilized by the pressure albeit for reasons different from those discussed in GGJ. The scaling with the plasma parameters predicted in [6], however, is identical with GGJ but there is a multiplying factor which is smaller by an order of magnitude, and therefore supersedes GGJ. This work is summarized in Section 4.4 and comments further on the stabilizing influence of the pressure.

\subsection{Sensitivity to the Assumed Pressure Profiles}

A recent theoretical study by Bishop et al. [7] concluded that the stability properties of tearing instabilities is so sensitive to details of the pressure profile in the immediate vicinity of the rational surface of interest that it is impossible to predict whether the mode will be unstable or not! In particular, Bishop considered the effect of a 'flat spot' in the pressure profile centered at the mode rational surface of interest and showed that if the

radial extent of the pressure perturbation was larger than the resistive layer width (which is the only situation of practical interest), then the stabilization effect predicted by GGJ is modified, essentially such that the resistive layer width is replaced by the macroscopic width of the flat spot and the stabilizing influence of the pressure is reduced. We had ourselves arrived at a similar conclusion independently, though empirically, by arbitrarily introducing narrow flat spots in our equilibrium pressure profiles in the immediate vicinity of the rational surfaces of interest. In this situation, we found computationally that the instability then persists into the range of experimental interest as illustrated in Fig. 4.3. Also, when $S$ is sufficiently large, the growth rates tend to the asymptotic $S^{-3 / 5}$ scaling predicted by narrow layer theory, identifying the instability as a tearing mode. Fig. 4.4 illustrates the corresponding pressure profiles used to calculate the latter equilibria. Flat spots in the pressure profile are, indeed, observed experimentally, though these are believed to be the result of the instability. Here we suggest that a flat spot is necessary to trigger the mode. While any number of 'hand-waving' mechanisms can be invoked that may produce such a condition (field errors, short wavelength electrothermal instabilities [8], etc.), here we shall simply regard the introduction of flat spots in the pressure profile as an artifice to remove the GGJ stabilization which theorists currently tend to regard as unimportant since it ignores additional fine scale physics such as kinetic effects, 

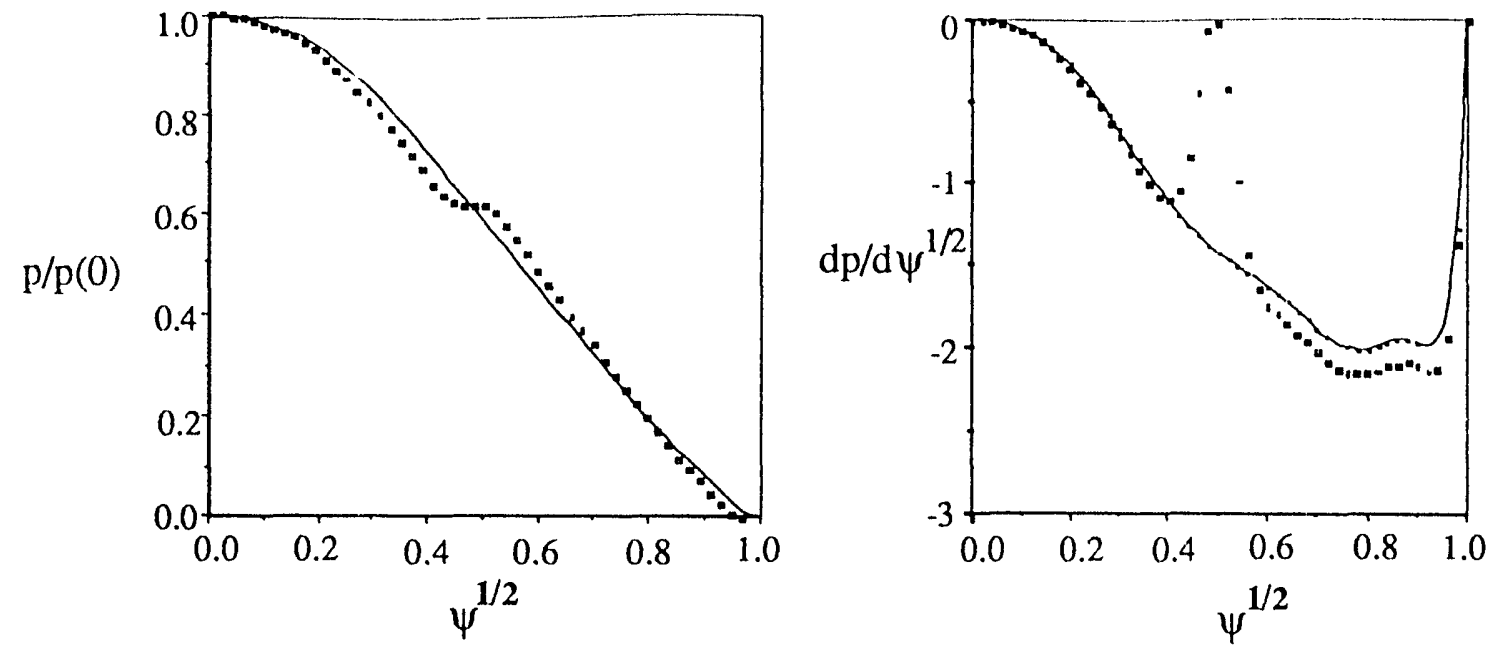

Fig. 4.4 Pressure and pressure gradient distributions illustrating the 'flat spots' which enhance the instability of the tearing modes. In these graphs, the continuous curves show the unperturbed pressure profiles while the symbols show the 'flat spots'.

equilibrium flows $[9,10]$ etc., which can significantly alter the effects of an extremely narrow resistive layer (which can be smaller than the ion Larmor radius, for example). This stabilizing influence of the pressure is, however, a valid prediction of the single fluid, resistive MHD model defined, for example in Coppi, Greene and Johnson [4], and presently implemented in the ARES code.

\subsection{Sensitivity to the Assumed Current Density Profile}

The previous Sections have highlighted the strong stabilizing influence of finite pressure on the tearing mode and the manner in which this effect can be mitigated by small scale details in the pressure profile. In the following, the pressure profile is always adjusted such that the pressure gradient is zero over some small radial width in the vicinity of the rational surfaces, as described above. Here, we shall gauge the sensitivity of these results to the assumed parameters and to study the effects of independent changes in the total pressure (keeping the profile shape fixed) and the q-profile. Since the data is obtained in numerical form, we have adopted the following procedure to alter the equilibrium current density profile, or more precisely the profile of the surface function $\langle\mathbf{J} \cdot \mathbf{B}\rangle /\langle\mathbf{B} \cdot \nabla \phi\rangle$ in a systematic manner. 

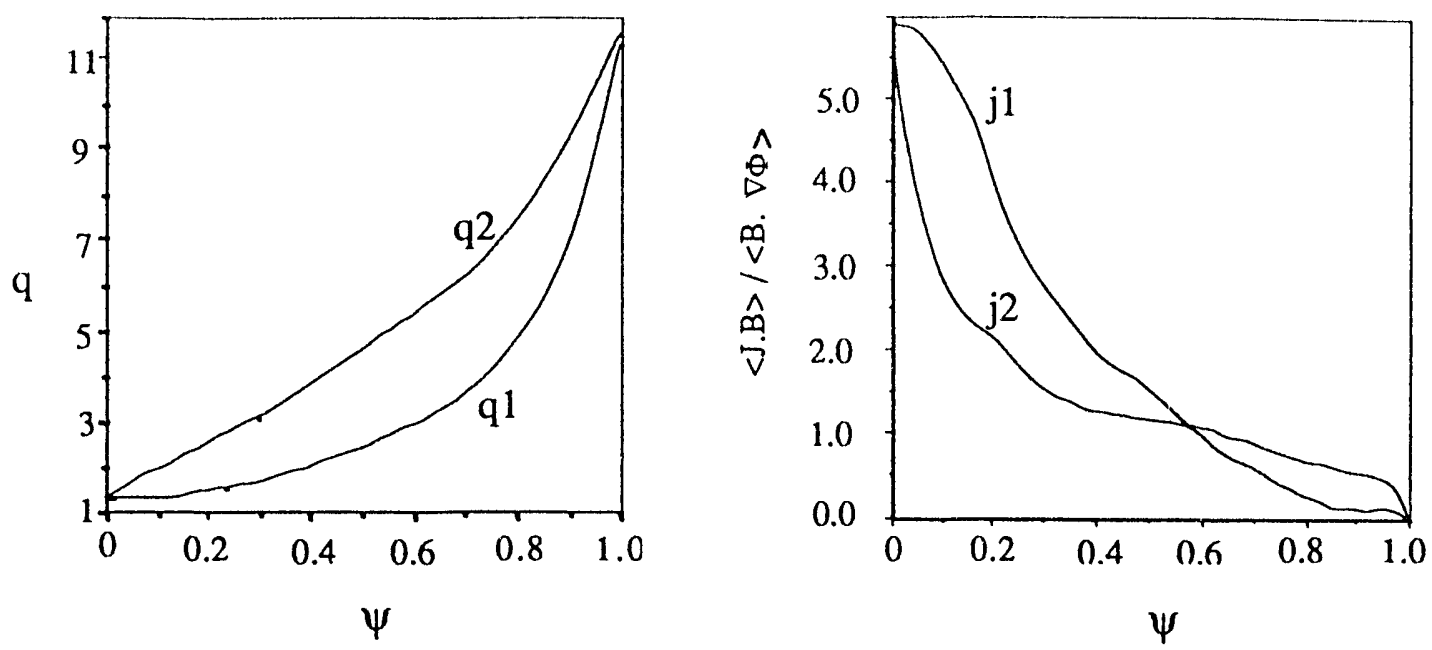

Fig. 4.5 q-profiles and corresponding current density distributions. The profile labeled $\mathrm{q} 1$ is that from shot \#26637 while q2 is obtained from shot \#46494 where the current was ramped up during beam heating, generating a broader current profile. These are used to generate a sequence of intermediate profiles.

The curve labeled q1 in Fig. 4.5(a) is the q-profile from shot 26637 given by TRANSP but with $q(0)$ increased to 1.25 ; the corresponding parallel current distribution is the curve $\mathrm{j} 1 \mathrm{in}$ Fig. 4.5(b). This q-profile was known to be tearing mode unstable from our previous calculations. Also shown in figs.4.5(a) and 4.5(b), and labeled q2, i2, are profiles from a different experiment (shot \#46494) in which the current was ramped up during the beam heating phase thereby generating a broader current distribution and a correspondingly steeper q-profile. Analysis of this latter shot shows that it is resistively stable. We then take linear combinations of $\mathrm{q} 1$ and $\mathrm{q} 2$ to generate a family of intermediate q-profiles using the inductance per unit length, $\mathrm{l}_{\mathrm{i}}$, to characterize the corresponding current density distributions, since this quantity can at least be estimated experimentally. Equilibria were obtained over a range of $\mathrm{l}_{\mathrm{i}}$, and these were subsequently analyzed for stability. Each equilibrium was shown to be stable to ideal modes with the 'wall on plasma' boundary condition. 


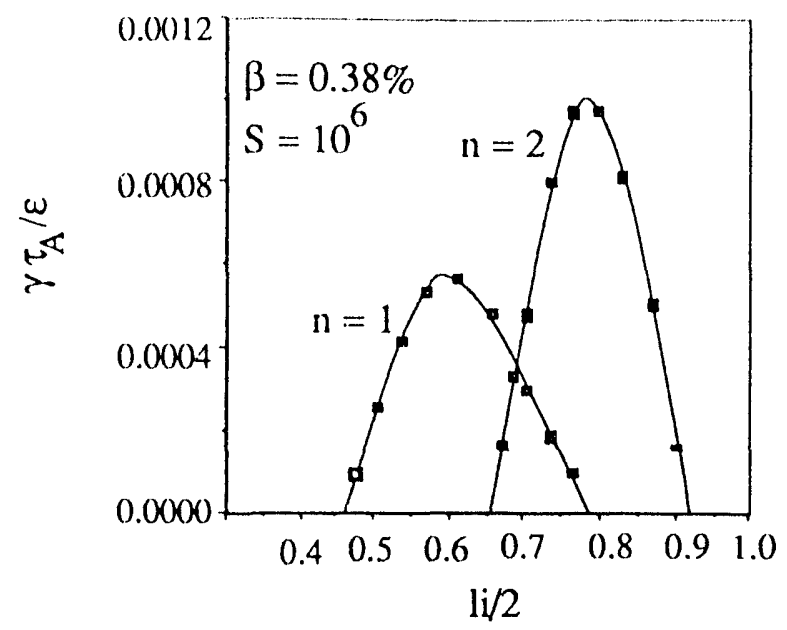

Fig. 4.6 Tearing mode growth rates as a function of li at constant pressure. Analysis of shot \#26637 varying the current density profile shows both $2 / 1$ and $3 / 2$ mode. The nominal value of li/2 output by TRANSP is 0.77 where the $3 / 2$ mode is strongly excited and close to the marginal point for the $2 / 1$ instability.
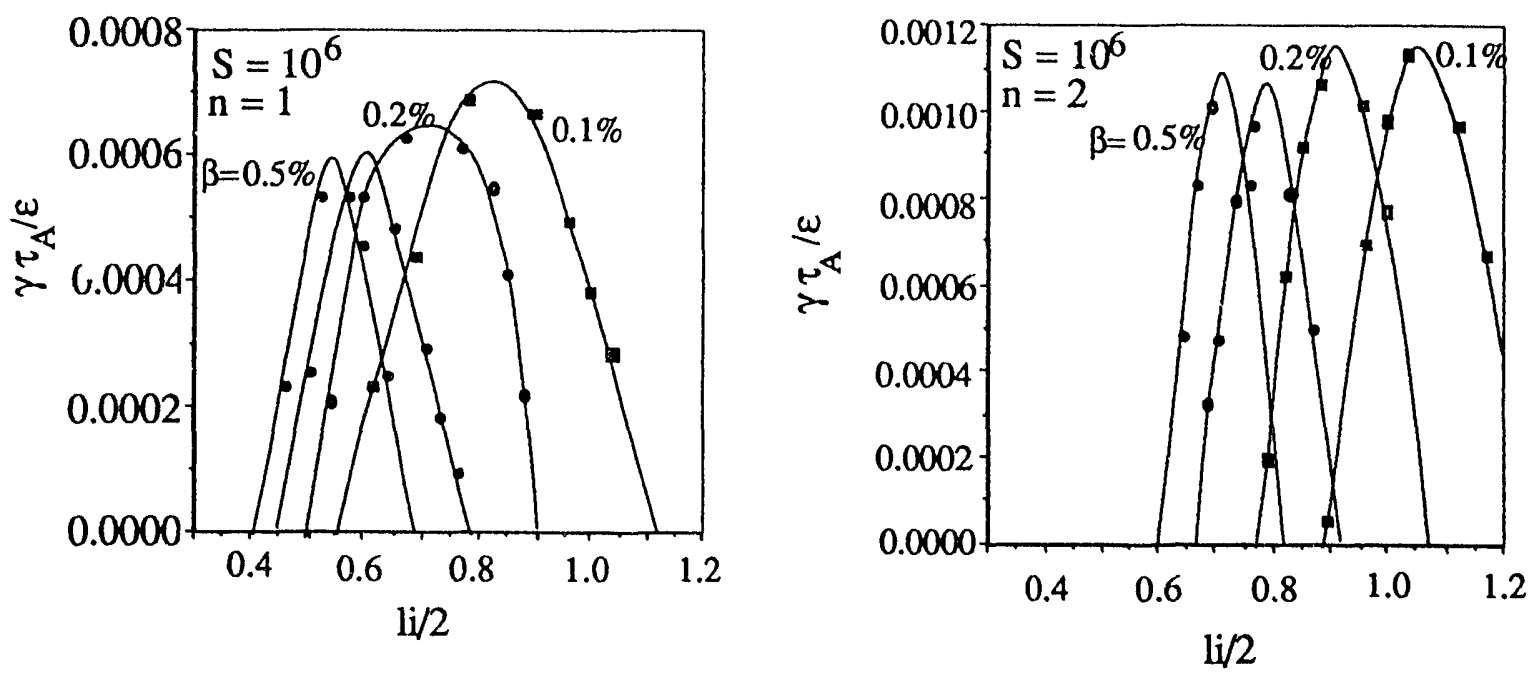

Fig. 4.7 Tearing mode growth rates as functions of li when the pressure is varied. Note that, as the pressure is increased the peaks shift to smaller values li and they tend to become narrower.

Fig. 4.6 shows the mode growth rates as a function of $l_{i}$ when $\beta=0.38 \%$, corresponding to the pressure in shot \#26637 at the time when the $3 / 2$ mode appeared. As $l_{i}$ is scanned at constant pressure we find both the $2 / 1$ and $3 / 2$ modes as before (Fig. 4.2) except that $q(0)$ is now held constant. Modes with toroidal mode number $n>2$ are not excited. The value of $1_{i} / 2$ calculated by TRANSP is 0.77 which, referring to Fig. 4.6 is close to the marginal point for the $2 / 1$ mode but in the range where the $3 / 2$ tearing mode is strongly excited, in agreement with the experimental observation. Fig. 4.7 shows how this 
diagram is alte:ed whe $a$ the pressure is varied. Note that as the pressure is increased, the peaks shift to smaller values of $l_{i}$ and become narrower. It is this effect which prompted the computational procedure adopted here, since changing the pressure while keeping the $q$ profile fixed can change $l_{i}$ substantially and can lead to erroneous conclusions.

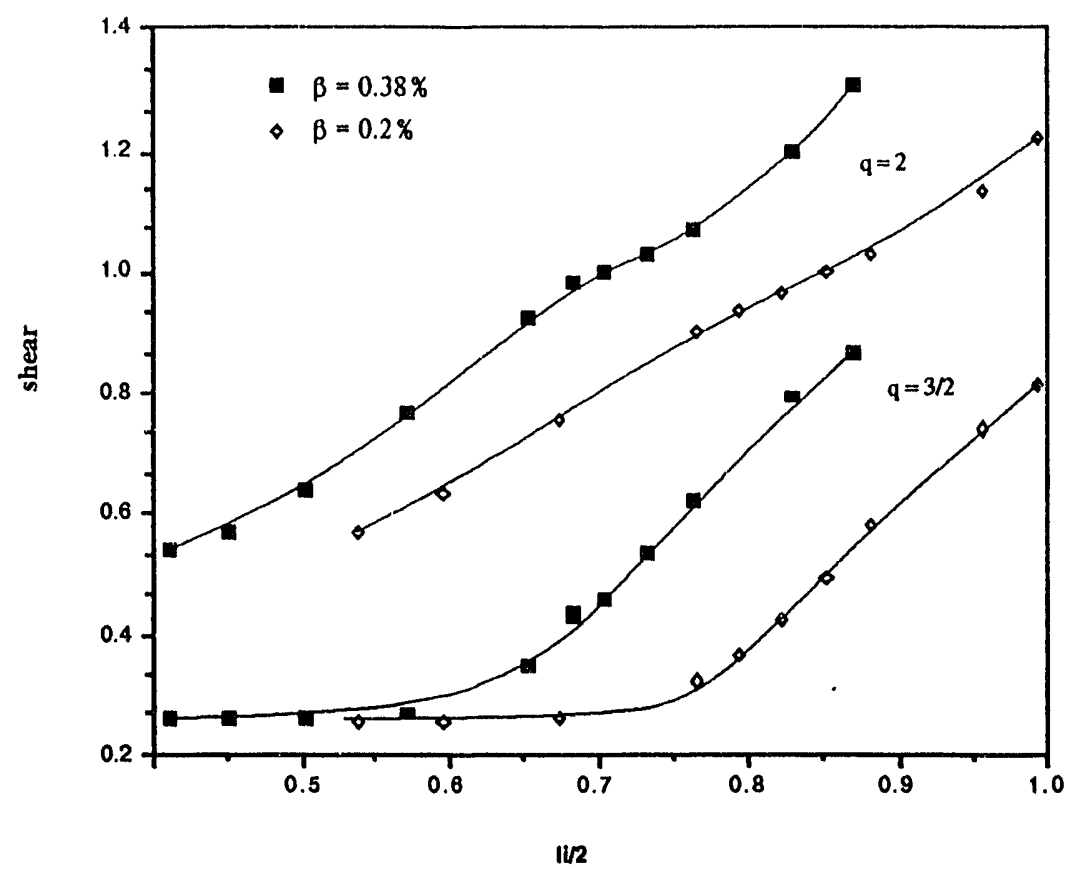

Fig. 4.8 Variation with li of the shear at $q=3 / 2$ and $q=2$.

Analytic theory shows that the shear ( $\equiv \mathrm{r} / \mathrm{qdq} / \mathrm{dr}$ ) evaluated at the rational surfaces is an important parameter in determining the growth rates of tearing modes. Thus, each calculation monitored the shear at the $q=3 / 2$ and $q=2$ surfaces and Fig. 4.8 shows how the shear changes with $l_{i}$. Thus increasing $l_{i}$, typically means increased shear at the rational surfaces. Using this information, Fig. 4.9 plots the mode growth rates as a function of the shear at various pressures. While, in this case, there is a weak dependence on the pressure, the graphs suggest that, for both modes studied here, the growth rates depend primarily on the shear at the rational surface. Note that there are bands of shear values where each mode is excited. Also, the stabilization that appears when the shear is large is not predicted by analytic theory. We believe that this behavior is a toroidal effect associated with the fact that as the current density becomes more peaked and $l_{i}$ (and hence the shear) increases, the rational surfaces move outward (i.e. the aspect ratio of the rational surfaces decreases). While this effect is not understood, it has been observed in other calculations and an analogous example appears in the literature [11]. 

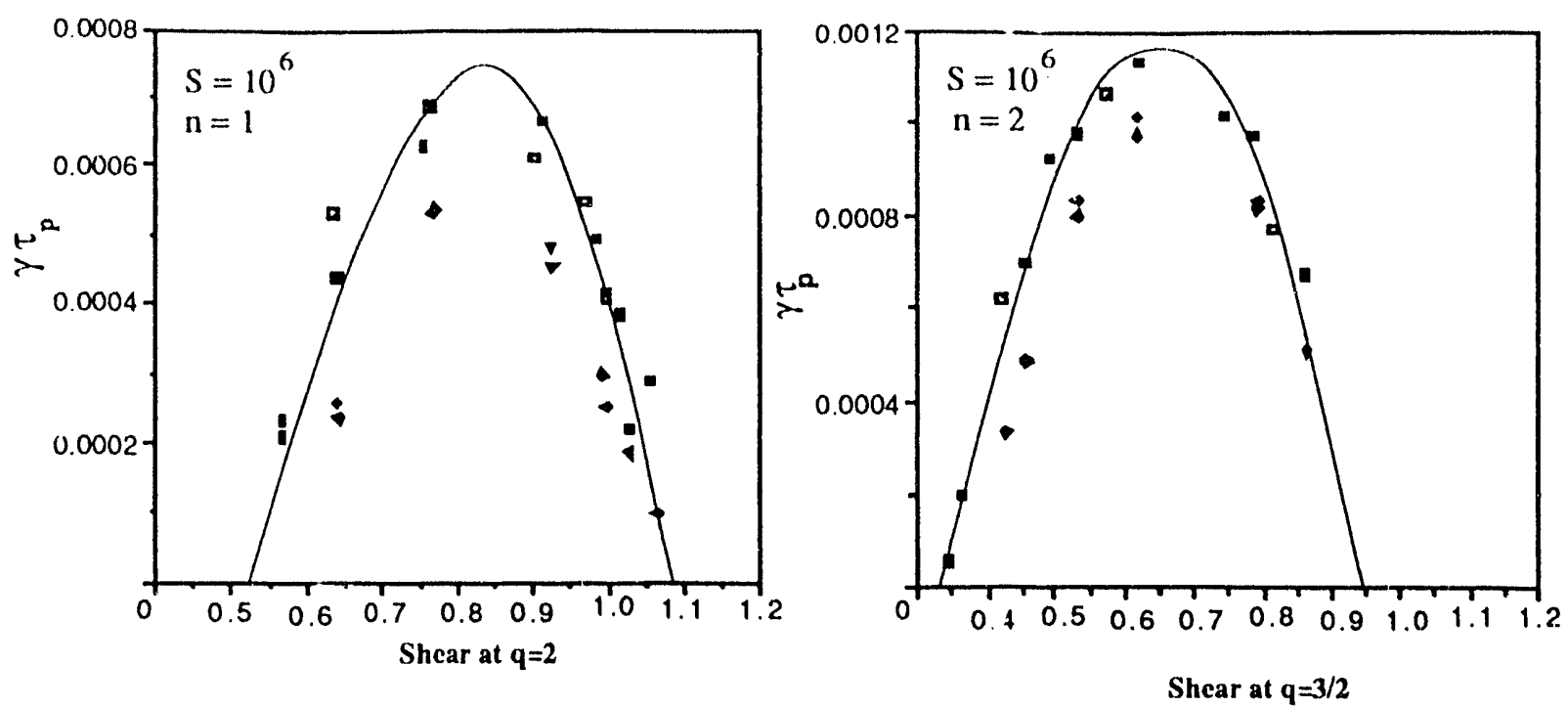

Fig. 4.9 Tearing mode growth rates as functions of the shear evaluated at $q=3 / 2$ and $q=2$.

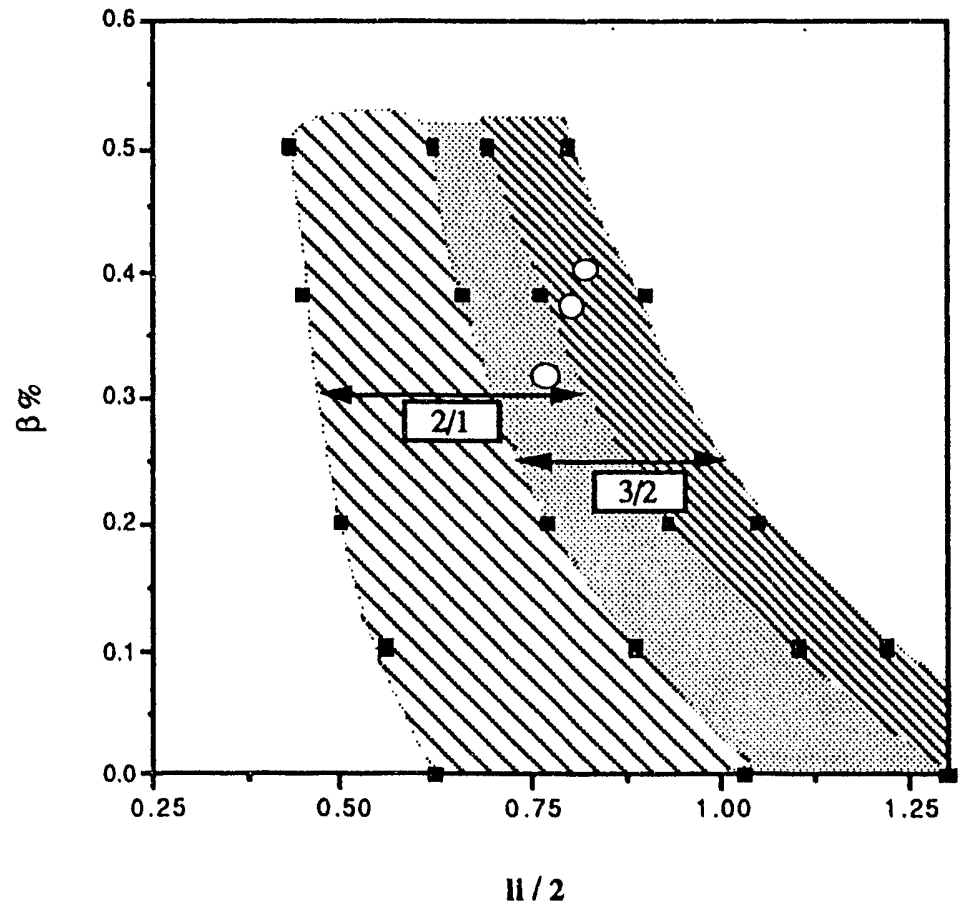

Fig. 4.10 Tearing mode stability diagram. The hatched areas show the regions where only one mode is excited. The open circles indicate the nominal parameters output by TRANSP. 
By fitting curves to the growth rate versus $l_{i}$ data at each pressure, we can estimate marginal stability points. Thus, the entire series of stability calculations is summarized in Fig. 4.10 which plots these marginal points in the $\left(l_{i}, \beta\right)$ plane. Note that there is no contradiction between this and the results shown in Fig. 4.2 at the constant value of $q(0)=1.25$ used to generate Fig. 4.10. The value of $q(0)$ is an additional parameter determining the shear at the rational surfaces. Referring to Fig. 4.10 , in this $\left(l_{i}, \beta\right)$ diagram there is a band where only the $3 / 2$ mode is excited, a second band at smaller $l_{i}$ where only the $2 / 1$ instability appears with a region of overlap where both modes are found. When $l_{i}$ is large enough the modes are stable. This diagram exhibits some of the qualitative features which we had set out to find. For example, the open circles in Fig. 4.10 indicate the locations of the three sets of supershot data considered here and it is tempting to claim agreement with the experimental observations. However, when we consider a much larger set of experimental data ( 100 supershots), estimating $l_{i}$ from the shift of the axis and the known $\beta$ p, we find that those shots which exhibited $2 / 1$ instability and those which were $3 / 2$ unstable occupy the same region of the $\left(l_{i}, \beta\right)$ diagram. Thus, even with the essential caveat of 'flat spots' in the pressure profile located exactly at the rational surfaces there is no convincing correlation between the predictions of tearing mode theory and the experimental observations.

\subsection{Theoretical Studies of the Tearing Mode}

The resistive MHD studies described above, particularly the importance of finite pressure stabilization of the tearing mode, have prompted a detailed re-examination of linear tearing instability theory. We participated in this study in collaboration with colleagues at Columbia University and General Atomics. This work is currently in preparation for

publication[ 6] where the mathematical detail will be presented fully. Here we shall simply summarize some relevant conclusions.

In this work the dispersion relation for resistive interchange and tearing modes [3] in cylindrical geometry was used to compare the two cases of zero and finite $\beta$ in detail. Note that, here we define $\beta=\Gamma \mathrm{p} / \mathrm{B}^{2}$ where $\Gamma$ is the adiabatic index. 'Zero $\beta$ ' then refers to the situation where the pressure tends to zero but the pressure gradient at the rational surface remains finite; in the terminology of ref. [12], this corresponds to 'infinite compressibility' where $\Gamma=0$. The analytic results were checked, in minute detail, with 
calculations using ARES for a specific cylindrical equilibrium described previously in the literature $[13,14]$. The equilibrium profiles used are defined by

$$
\begin{aligned}
& q(r)=1.6\left(1+r^{2} / 0.64\right), \\
& p(r)=0.25\left(0.001+0.028 r^{2}-0.059 r^{4}+0.03 r^{6}\right)
\end{aligned}
$$

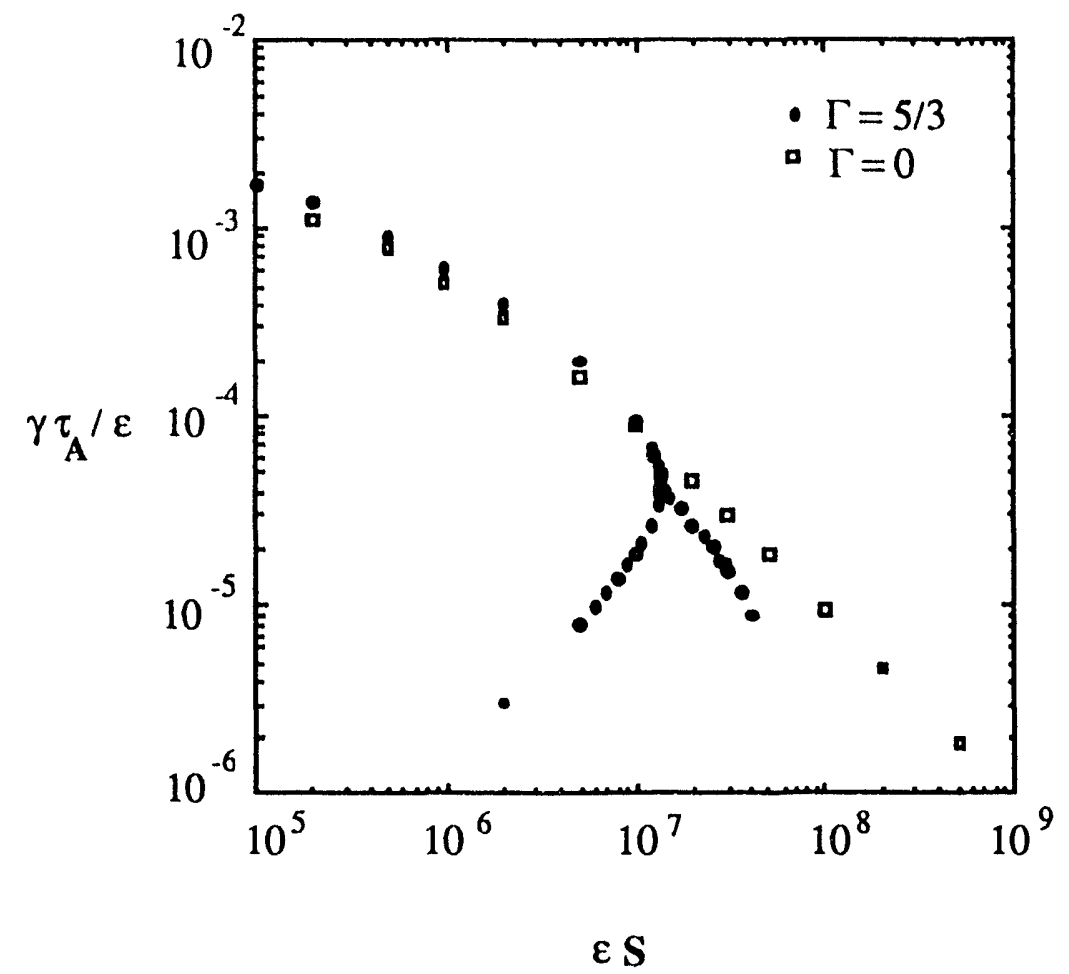

Fig. 4.11 Variation of the growth rate with the Lundquist number in cylindrical geometry. The cylindrical equilibrium is defined by $p=0.25\left(0.001+0.28 r^{2}-0.059 r^{4}+0.03 r^{6}\right), q=1.6(1+r 2 / 0.64)$, and the aspect ratio is 5 . The open symbols refer to the case $\Gamma=0$ while the solid symbols have $\Gamma=5 . / 3$.

where $\mathrm{r}$ is the radial coordinate, $0 \leq \mathrm{r} \leq 1$, and the pressure profile is such that $\mathrm{dp} / \mathrm{dr}$ is positive at the resonant surface $q=2$ to stabilize the resistive interchange mode. Both the numerical calculations, shown in Fig. 4.11, and analytic theory show that there is a departure from the tearing mode scaling, $\eta^{3 / 5}$, of the growth rate as the resistivity, $\eta$ ( $\equiv$ $1 / S)$, becomes smaller than some critical value even at zero $\beta$. At small $S$ there are two modes which ultimately coalesce as $S$ is increased. For the particular equilibrium considered here this transition occurs when $S=1.05 \times 10^{7}$. Beyond this critical value the 
growth rate scales as $S^{-1}$ (i.e. with the resistivity, $\eta$ ) when the adiabatic inde:, and hence $\beta$, is zero. When $\Gamma=5 / 3$ the corresponding eigenvalues are close to the $\Gamma=0$ case at small $S$ but become complex (oscillatory growth) above the critical value of $\mathrm{S}$ with the real part tending to zero even more rapidly than $S^{-1}$. It is shown in [6] that finite pressure is effective only in this latter regime. Since the fundamental basis of linear tearing instability theory depends on the existence of modes with growth rates scaling as a fractional power of $\eta$, it is argued that the regime where the growth rate scales as $\eta$ has no physical relevance; the linear tearing mode is essentially stabilized since it cannot be distinguished from resistive diffusion. It is also argued that in this regime, other hydrodynamic effects, such as mass flows and viscosity, cannot be neglected and would alter the stability properties of the system.

Defining a normalized growth rate

$$
\mathrm{Q}=\frac{\gamma \tau_{\mathrm{A}}}{\varepsilon}(\varepsilon S)^{1 / 3}\left(\frac{1}{\mathrm{n}} \frac{\mathrm{q}}{\mathrm{aq}^{\prime}}\right)^{1 / 3}
$$

and the equilibrium Suydam parameter

$$
D_{s}=-\frac{2 q^{2}}{B^{2} r_{s} q^{2}} \frac{d p}{d r}
$$

it is shown that $\mathrm{Q} /\left|\mathrm{Ds}_{\mathrm{s}}\right|^{2 / 3} \cong 1$ is a critical value above which finite pressure has no effect and below which it is stabilizing, though irrelevant from a practical point of view. This leads to a critical Lundquist number

$$
(\varepsilon S)=\frac{1}{\pi^{3}} \frac{\left(\Delta^{\prime}\right)^{3}}{|D s|^{5 / 2}}\left(\frac{1}{\mathrm{n}} \frac{\mathrm{q}}{\mathrm{aq^{ \prime }}}\right)
$$

above which linear theory predicts that tearing modes are effectively stabilized. Interestingly, the parametric dependencies in this formula for the critical value of $\mathbf{S}$ are identical with those derived by GGJ. The leading coefficient, however, is smaller than that in GGJ by an order of magnitude and therefore supersedes the latter. Inserting numerical values in this formula predicts the computational transition point if $\Delta^{\prime}=15$ for the model equilibrium considered; this value was subsequently confirmed using a cylindrical $\Delta^{\prime}$ code, thereby generating some confidence in the correctness of the theoretical calculation. 
An interesting and very relevant conclusion drawn in $|6|$ is that, when the Lundquist number exceeds the critical value given above, the classical theory of the tearing mode is inadequate. Thus, for example, by comparing the mode growth time with the that required to traverse the resistive layer it is estimated that even the perpendicular flows associated with resistive diffusion cannot be neglected. This mass flow can be either stabilizing or destabilizing [14].

\subsection{Conclusions}

The main conclusions of these studies of the tearing mode in TFTR plasmas can be summarized as follows:

The finite pressure stabilization effect described by Glasser, Greene and Johnson is very strong in supershot plasmas where $\beta_{p}$ is large $(\sim 2)$.

- The stabilizing influence of the pressure is sensitive to small scale details of the profile.

When this stabilizing influence is removed by arbitrarily reducing the pressure gradient in a narrow region in the vicinity of the rational surfaces of interest, there is some qualitative agreement with experiment. Specifically, we find :

(1) bands of shear values which are linearly unstable to tearing modes;

(2) conditions are found where either the $2 / 1$ or $3 / 2$ tearing instability is excited with a region of overlap where both modes appear;

(3) the low $\beta$ supershots considered here lay in the region where the instability is predominantly $3 / 2$ and near marginal for the $2 / 1$, superficially in agreement with experiment.

- When the computational predictions are compared with a larger set of experimental data there is no convincing correlation with experiment.

- Theoretical studies prompted by these computational results show that the effective stabilization of the tearing mode occurs at a value of the Lundquist number that is an order of magnitude smaller than that predicted by Glasser, Greene and Johnson. 
- The analytic studies suggest that, particularly in the very high temperature supershot plasmas in TFTR, other hydrodynamic effects cannot be ignored in the regime where the pressure stabilizes the tearing mode. Mass flow, for example, can be important even at the resistive diffusion speed and this can be either stabilizing or de-stabilizing.

[1] Furth, H.P., Killeen, J. and Rosenbluth, M.N., Phys Fluids, 6, (1963), 459.

[2] Connor, J.W., Cowley, S.C., Hastie, R.J. and Martin, T.J., 12th Int. Vonf. on Plasma Physics and Controlled Nuclear Fusion Research, IAEA, Nice, 1988, paper IAEA-CN-50/D-1-4

[3] Coppi, B,. Greene, J.M., and Johnson, J.L., Nuclear Fusion, 6, (1966), 101

[4] Glasser, A.H., Greene, J.M. and Johnson, J.L., Phys. Fluids, 18, (1975), 875

[5] Hastie, R.J., Sykes, A., Turner, M. and Wesson, J.A., Nuclear Fusion, 17, (1977), 515

[6] Iacono, R., Bhattacharjee, A., Ronchi, C., Greene, J.M., and Hughes, M.H., in preparation, to be submitted for publication in Phys. Fluids B

[7] Bishop, C.M., Hastie, R.J., Connor, J.W. and Cowley, S.C., Plasma Phys. and Controlled Fusion, 33, (1991), 389.

[8] Haines, M.G.,and Tomimura, A., J.Plasma.Phys., 22, (1979), 397

[9] Dobrott, D., Prager, S.C. and Taylor, J.B., Phys. Fluids, 20, (1977), 1850

[10] Pollard, R.K. and Taylor, J.B., Phys. Fluids, 22, (1979), 126

[11] Hastie.R.J., Hender, T.C., Carreras, B.A., Charlton, L.A., and Holmes, J.A., Phys.Fluids. 30, 1987, 1756

[12] Glasser, A.H., Phys. Fuids B, 3, (1991), 2078

[13] Ryu, C.M. and Grimm, R.C., J.Plasma. Phys., 32, (1984), 207

[14] Izzo, R., Monticello, D.A., DeLucia, J., Park, W. and Ryu, C.M., Phys. Fluids, 28, (1985), 903 
-

-

-

-

-

-

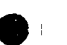

-

-

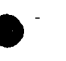




\section{MHD ANALYSIS OF HIGH $\beta$ SUPERSHOT PLASMAS}

The previous Section considered the possibility that the instabilities which occur in supershot plasmas are purely resistive in character. In this Section and in the next, we investigate the role of ideal MHD modes. In particular, we have carried out a detailed study of both large and small toroidal mode number pressure driven 'ballooning' modes, where one helical harmonic is coupled to its sidebands. This includes the so called 'infernal' modes [4], first predicted theoretically by Zakharov [1], which are essentially low-n ballooning modes made unstable by weak shear. In standard ballooning theory, valid only for strong shear, the largest $\mathrm{n}$ modes are the most unstable and as $\mathrm{n}$ decreases the growth rate also decreases [2]. When the shear is small this theory is no longer valid and low-n modes can become the more unstable. A weak shear extension of ballooning theory was first described by Hastie and Taylor [3]. In a computational study Manickam et al. [4] coined the name 'infernal mode' and demonstrated that these low-n instabilities can be unstable well below the limiting beta determined by high- $n$ ballooning modes.

In this Section we consider the MHD stability properties of shots \#35782 and \#55851 which are among the best supershots achieved, with $\beta$ reaching $\sim 1 \%$ before the appearance of MHD activity. It is suggested that the infernal mode, or resistive modifications of this mode, are plausible candidates for the observed instabilities in \#35782. In shot \#55851, where the current density is more peaked, we conclude that regular, low-n, ballooning modes are responsible. As before, we use numerical profile data obtained directly from TRANSP to generate equilibria, using the method described in Section 3.5 to alter the current distribution near the center.

\subsection{Analysis of Shot \#35782}

During the last few years, the performance of supershots in TFTR has continued to improve. Total currents of up $2 \mathrm{MA}$ and volume average beta close to $1 \%$ have been achieved. This has been mainly due to improved cleaning of the machine from the introduction of more extensive carbon limiters and boronization. Accompanying the increase in performance has been an increase in the frequency of occurrence of MHD activity. Previously when the machine operated at betas of $\sim 0.5 \%$, approximately $50 \%$ of the shots had MHD activity severe enough to degrade their performance; currently, $90 \%$ of the shots exhibit MHD activity. Although supershots have improved in general, MHD activity can still severely affect performance. In the best cases, MHD is associated with a 
drop off in neutron yield, and in the worse cases, a beta collapse and minor disruption. Figure 5.1 shows toroidal beta vs time for supershot, \#35782. After 4 seconds, $n=2$ MHD activity appears causing beta to decrease. This particular shot also had a carbon bloom which ultimately destroyed the plasma.

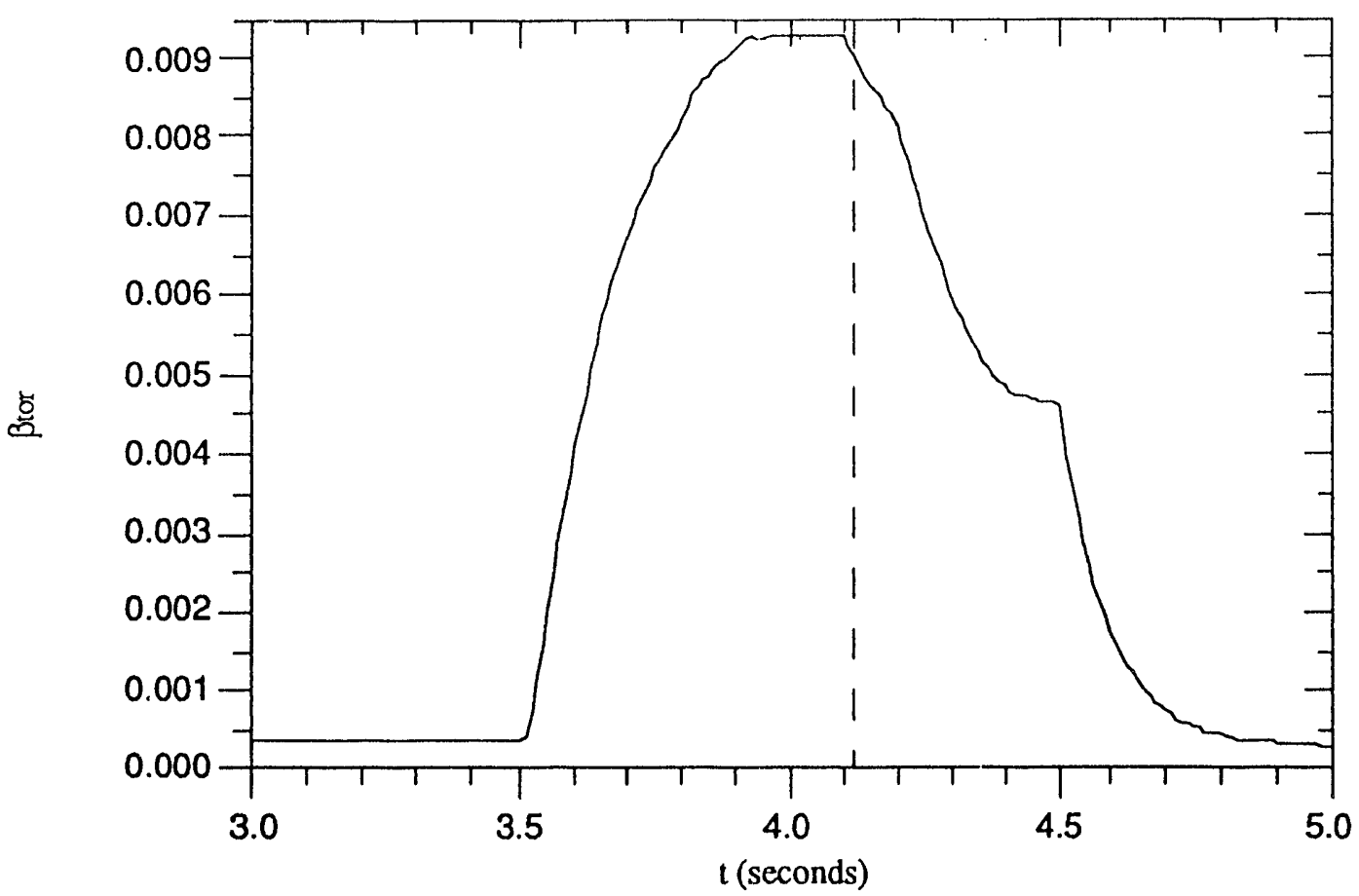

Fig. 5.1. Toroidal beta vs time for TFTR Shot\#35782. The vertical line indicates the time at which profiles were selected for analysis.

Most of the MHD activity observed in the high beta regime is associated with $\mathrm{n}=2$, though modes with $n=1,3$ and 4 are also seen. The modes are often pure $n=1,2,3$, or 4. However, a higher $n$ number sometimes transitions to lower $n$. The experimental data also suggests that perhaps more than one type of $\mathrm{MHD}$ activity may be occurring since the mode structures vary from shot to shot.

The fact that the frequency of MHD activity increases at higher $\beta$ suggests that the modes are pressure driven. (These shots also have higher current so current driven modes cannot be ruled out entirely.) The qualitative features of the mode activity also suggests that the instabilities could be infernal modes albeit modified by small, but finite, resistivity necessary to produce the parallel electric field required for island formation. Some 
pertinent features include: (a) the instabilities occur more frequently as $\beta$ is increased; (b) only a single mode is observed; (c) the pressure profiles in TFTR are highly peaked on axis. Analysis shows that these profile can extend into the transition region between the 1st and 2nd region of stability where infernal modes are expected. Another hint is that the axial value of $q$ is predicted by TRANSP to increase during the experiment until the instability begins as shown in Fig. 5.2. This reduces the shear in the center creating conditions liable to excite infernal modes.

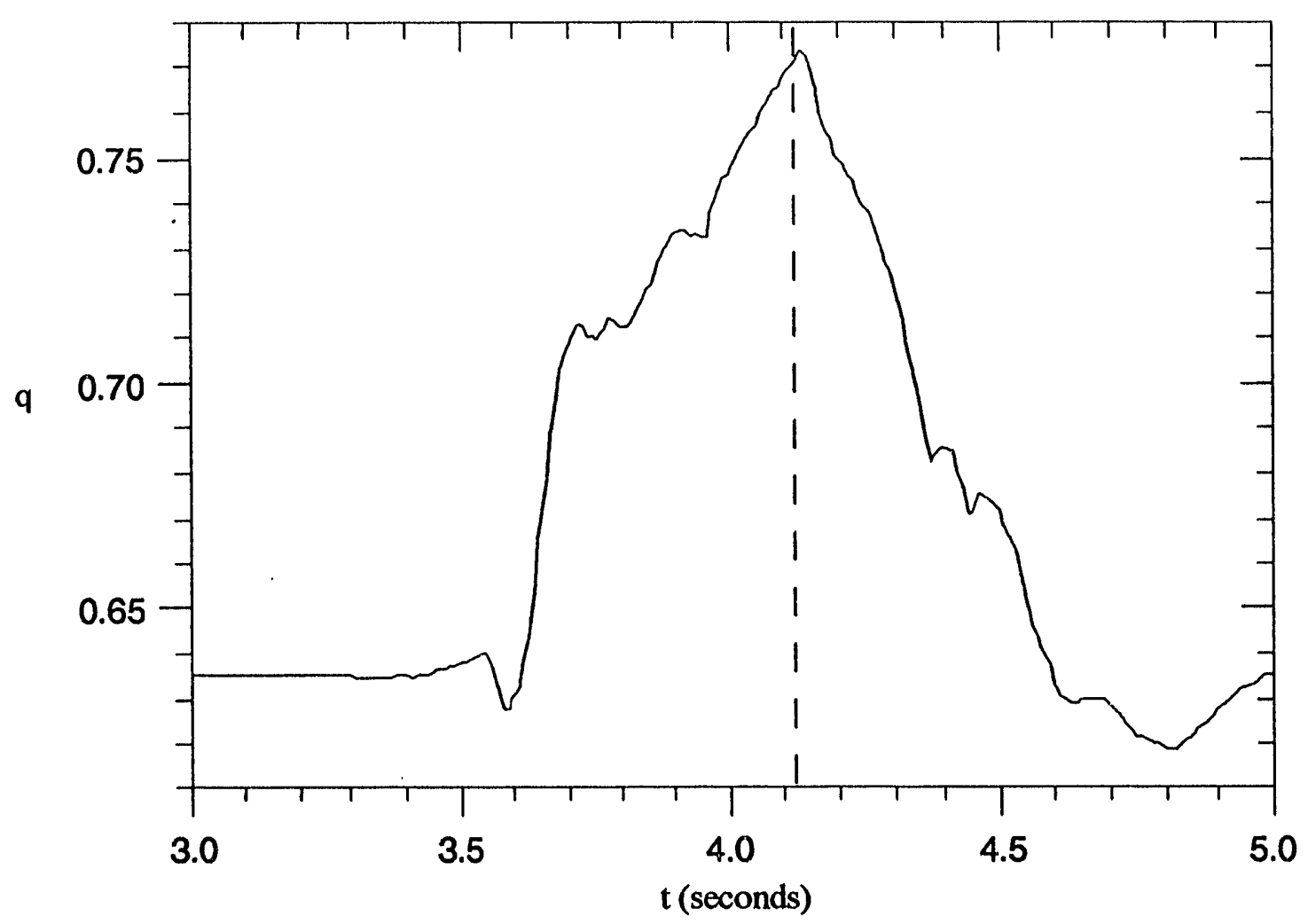

Fig. 5.2. Safety factor, q, on axis vs time for Shot \# 35782 predicted by TRANSP.

\subsubsection{Ideal Stability of Shot $\# 35782$}

Infinite-n ballooning stability is calculated using the ballooning code [5], described in Section 3.3, with 1st order corrections to changes in the equilibrium such that the 1st and 2nd stability boundaries can be calculated. Figure 5.3 shows the ballooning stability for 
shot \#35782 taken at $t=4 \mathrm{sec}$ for various values of $q(0)$. The curve shows the equilibrium pressure gradient and the shaded region indicates the region of instability. Stability to high$\mathrm{n}$ ballooning modes is achieved when $\mathrm{q}(0) \geq 1.21$. With some slight modification of the pressure profile, however, the equilibrium would be ballooning stable for $q(0) \geq 1.1$. Since the type of MHD activity observed in the experiment is composed of only a single toroidal mode number, ballooning modes are an unlikely candidate since more than one $\mathrm{n}$ number would be expected. In addition, the fact that tokamaks such as DIII-D and TFTR operate at beta limits near the Troyon limit [6] suggests that the pressure profile tends to assume an optimum shape for ballooning.
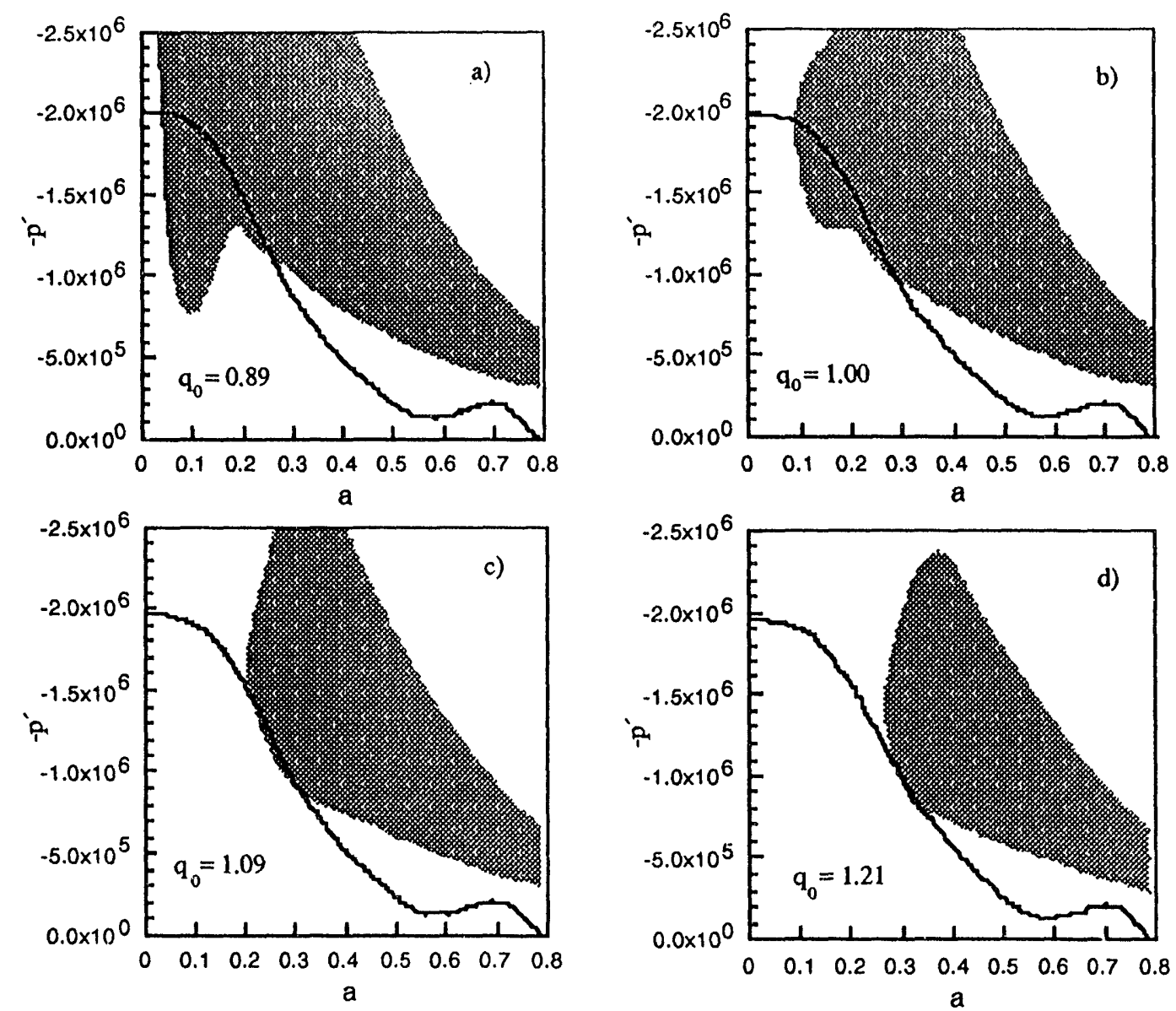

Fig. 5.3 Critical pressure gradients (units $\mathrm{Pa} / \mathrm{Wb} / 2 \pi$ ), defined by the boundary of the shaded regions, and equilibrium pressure gradient versus normalized $\psi$ for different $q$ profiles. a) $q(0)=0.89$, b) $q(0)=1.00$, c) $q(0)=1.09$, d) $q(0)=1.21$. 
In Fig 5.3(d) there is no stability limit to the pressure gradient in the center thereby providing access to the second region. The pressure profile in Fig. 5.3(d) extends up into what is termed the transition region between the first and second region of stability. Infernal modes are almost always found in this region.

Although for $q(0)>1.1$ the equilibrium is stable to high-n ballooning modes, a check of low-n stability shows that the equilibrium is indeed unstable to ideal infernal modes. The ideal growth rate vs $q(0)$ is shown in Fig. 5.4 for $n=2,3$ and 4. The particular mode that goes unstable depends on the values of $q(0)$ and $\beta$. There is a critical value of $\beta$ for the onset of these modes as in ballooning modes. For the $n=2$ mode $\beta>$ $0.65 \%$ for instability. For a given $q(0)$, typically only a single $\mathrm{n}$ number is unstable. This behavior is characteristic of infernal modes.

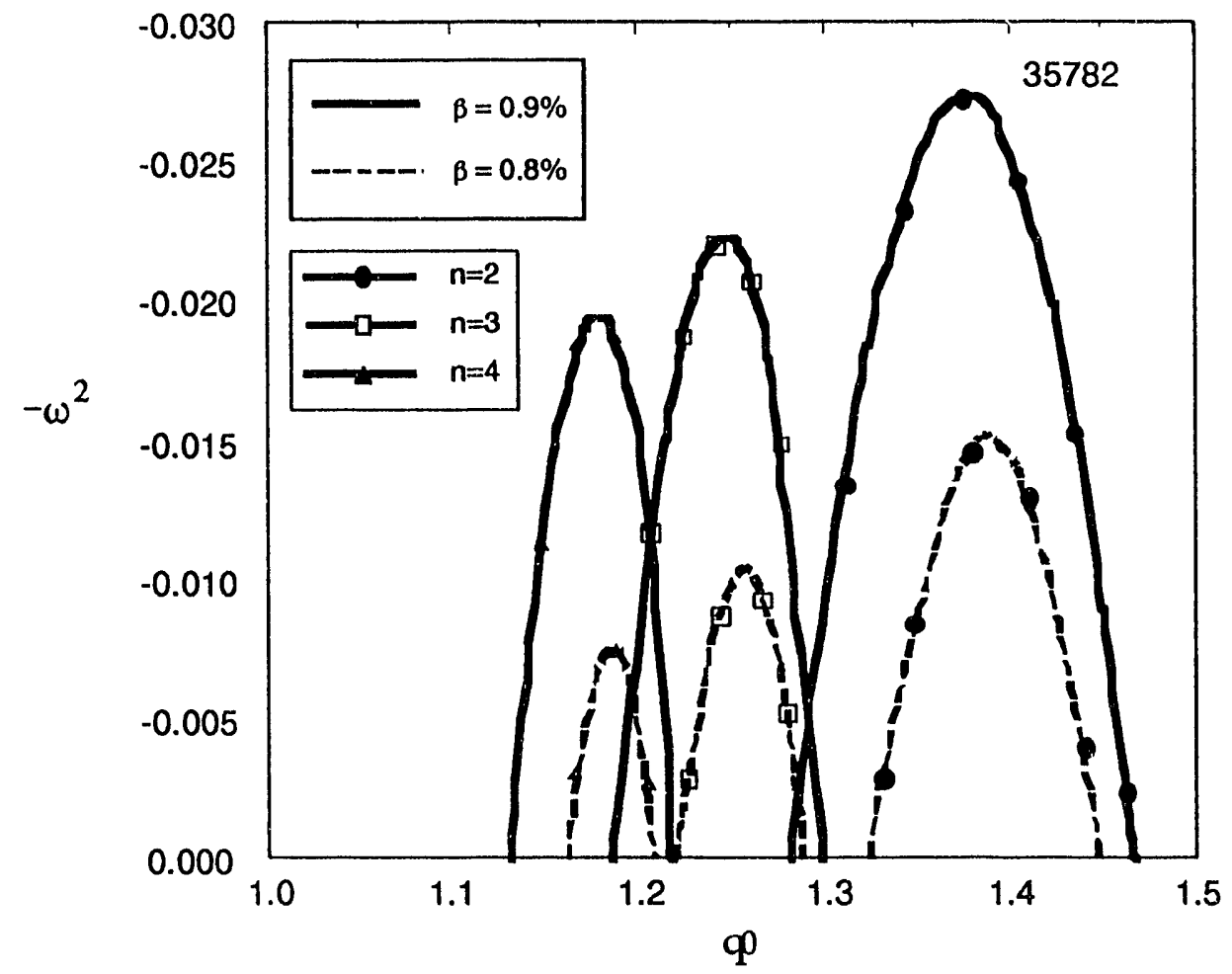

Fig. 5.4 Unstable eigenvalues vs $q(0)$ for $n=2,3,4$ Infernal modes for $\beta=0.8 \%$ and $0.9 \%$. 


\subsubsection{Resistive Stability}

Three regimes of infernal modes were identified by Charlton and Hastie [7,8] - (a) an ideal infernal mode, (b) surrounded by a mode having a scaling of $\gamma \sim S^{-3 / 13}$, termed a resistive infernal mode, (c) surrounded by a tearing mode region. The resistive stability for high beta TFTR supershots was examined using the ARES code. Fig. 5.5 shows growth rate vs $q(0)$ for the $n=2$ mode for both the ideal case and for $S=10^{7}, 10^{8}$. Here $S$ is the ratio of the resistive time $\left(\tau_{R P}=\mu_{0} \mathrm{a}^{2} / \eta\right)$ to the poloidal Alfven time $\left(\tau_{H P}=\sqrt{\mu{ }_{0} r_{m}} R_{0} / B_{0}\right)$. Typical $S$ values for TFTR are in the range of $10^{8}-10^{9}$. The plasma now becomes resistively unstable at a lower value of $q(0)$.

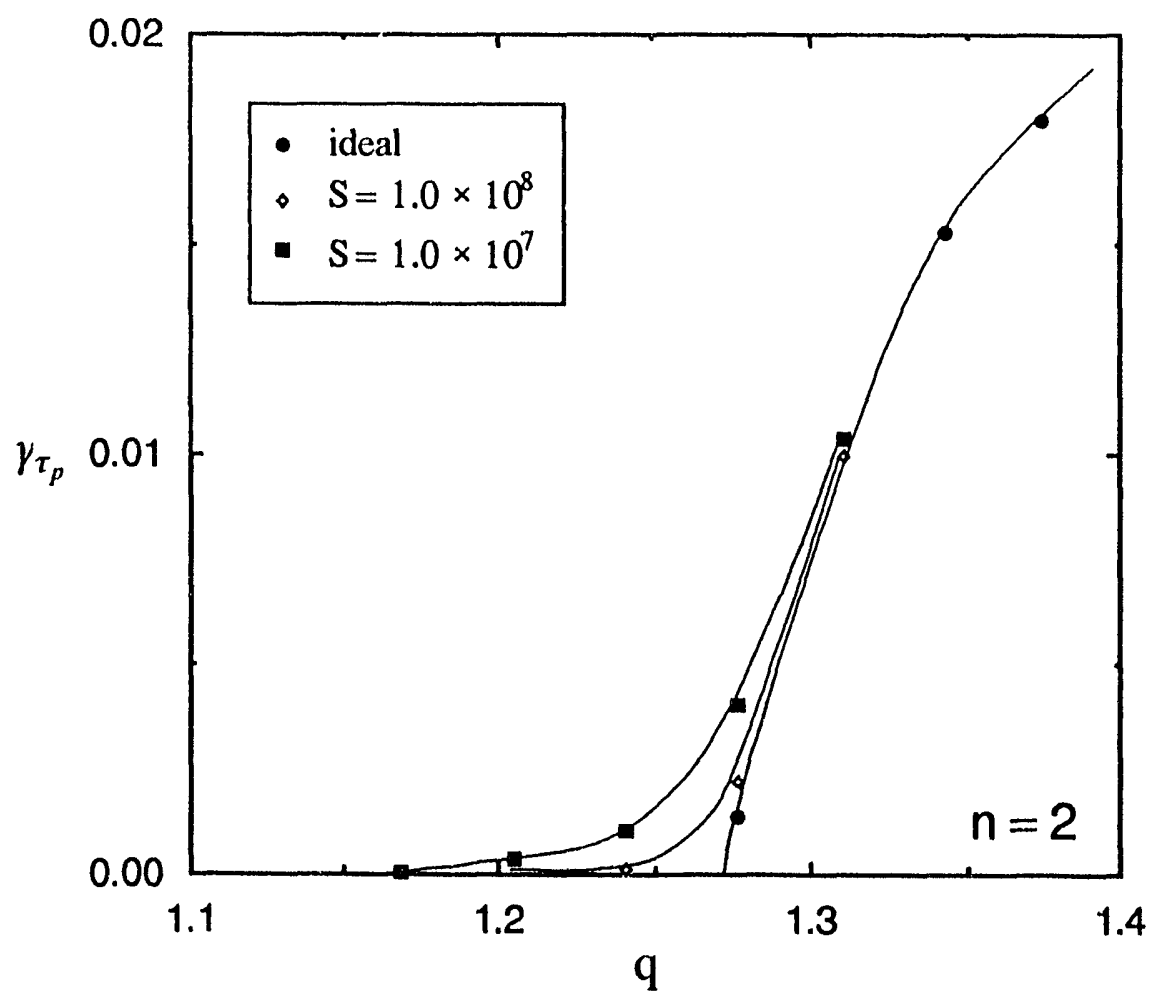

Fig. 5.5 Growth rate vs $q(0)$ for ideal and resistive $n=2$ modes.

As predicted by theory, it was found that the mode changes character as it goes from a regime where it is only resistively unstable to one where it is ideally unstable. At lower values of $q(0)$ in the range of 1.2 to 1.27 , the mode is essentially a tearing mode. Figure 5.6 shows the growth rate vs $S$ for $q(0)=1.24$ and $\beta=0.9 \%$. It can be seen that $\gamma$ $\sim S^{-3 / 5}$, characteristic of tearing modes. In addition, the eigenfunction structure is that of a 
tearing mode, highly localized about the mode rational surface. At the point where the ideal mode becomes unstable, but is still dominated by resistivity, the mode changes to a resistive infernal mode with the behavior predicted by Charlton and Hastie as shown in Fig. 5.7. Here the growth rate follows a $\gamma \sim S^{-3 / 13}$ scaling. The eigenfunction is still centered around the mode rational surface but is less localized than the tearing mode. Eventually the mode becomes ideally unstable and becomes much more global in nature.

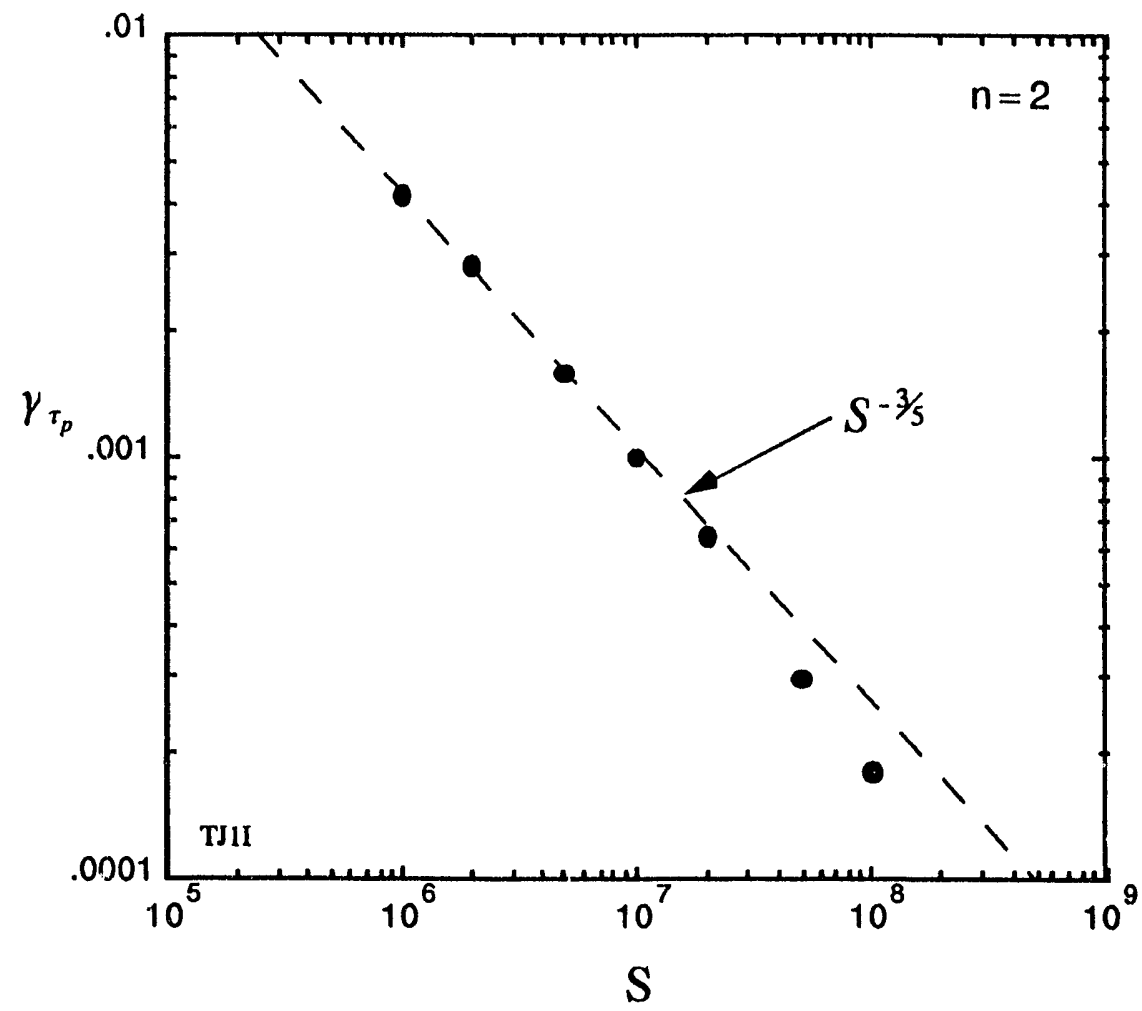

Fig. 5.6 Growth Rate vs $S$ for $\beta=0.90 \%, q(0)=1.24$ showing typical tearing mode behavior. 


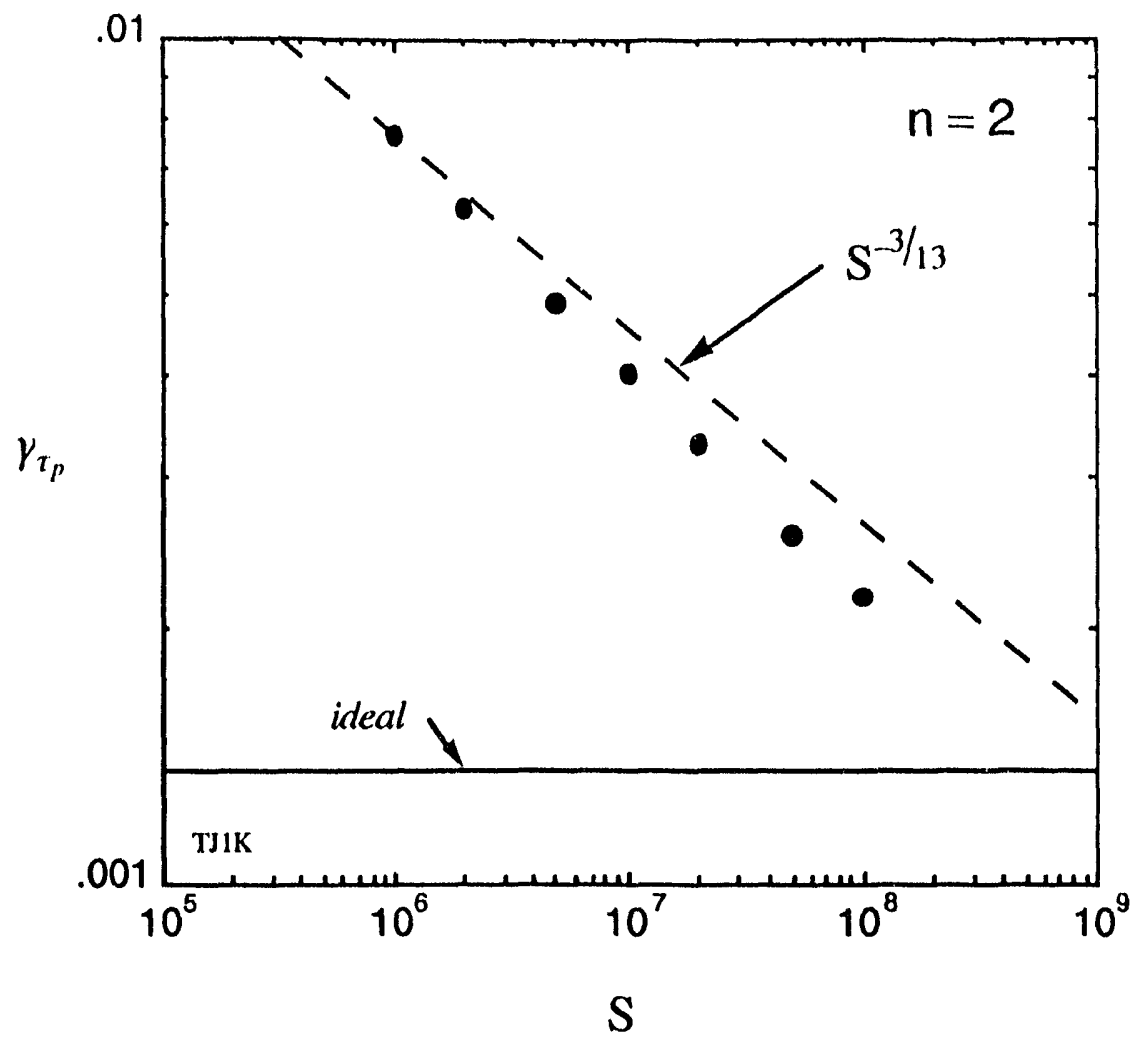

Fig. 5.7 Growth rate vs $S$ for $\beta=0.9 \%, q(0)=1.28$ showing resistive infernal mode behavior.

Unlike Charlton and Hastie, we found the resistive infernal mode behavior only when the ideal mode was already slightly unstable. The band of resistive infernal mode behavior was found to be quite narrow.

Figure 5.8 shows unstable regions in $(\beta, q(0))$ space for the $n=2,3$ and 4 modes. Since both $\beta$ and (presumably) $q(0)$ increase during the experiment, the $n$ number of the observed MHD activity will be determined by which unstable region the evolving equilibrium intersects first. Most of the MHD activity seen in the experiment is relatively benign, in the sense it does not result in catastrophic loss of the plasma. We would expect that this type of MHD activity is most likely due to the tearing mode. Some experiments observe a more rapid beta collapse which may correspond to an ideal mode which has a larger growth rate and is more global in nature. The region where the $n=2$ mode is unstable is larger than those for $n=3$ and $n=4$ which may account in part for the preponderance of $n=2$ modes observed experimentally. Still, in Fig. 5.8 the range of $q(0)$ necessary for this mode to occur is quite narrow and from this picture one would expect to 
see $n=3$ and $n=4$ more frequently than is seen in the experiments. This may be explained by the q profiles used for this study. For $q(0)<1.2$ the equilibrium was beginning to be ballooning unstable, which has the tendency to make the higher $\mathrm{n}$ modes more unstable. For flatter $q$ profiles, we would expect the $n=3$ and $n=4$ to be more stable, leaving the $n$ $=2$ mode dominant. Although not shown in Fig. 5.8, the $n=1$ mode is also unstable for high enough $q(0)$.

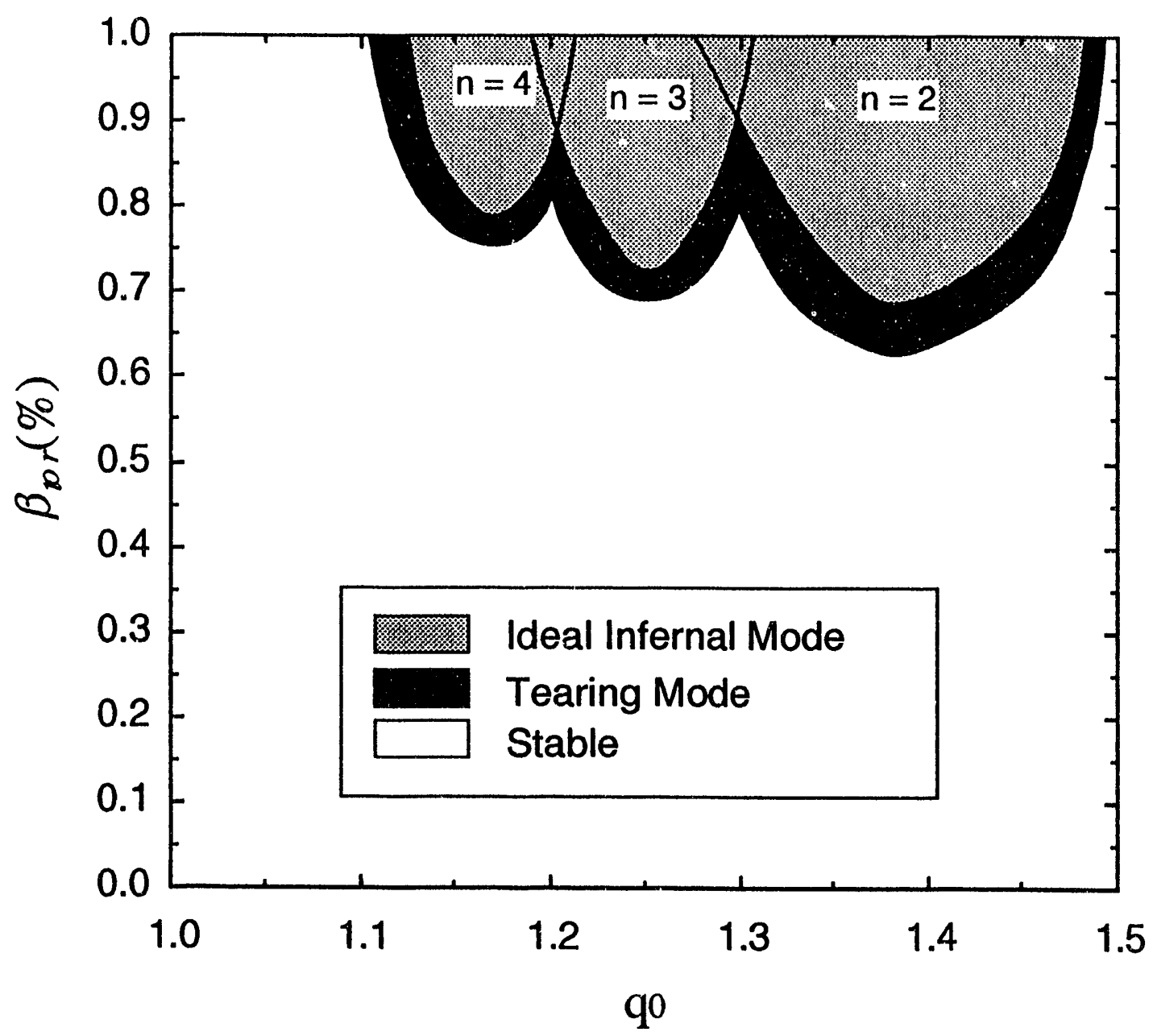

Fig. 5.8. Stability diagram for ideal Infernal mode and associated tearing mode. 


\subsection{Analysis of Shot \#55851}

Although most high beta supershots have some kind of MHD activity, not all end with a beta collapse. An example is shot \#55851 which is one of the best supershots obtained in TFTR. Figure 5.9 shows the time evolution of the toroidal beta for shot \#55851.

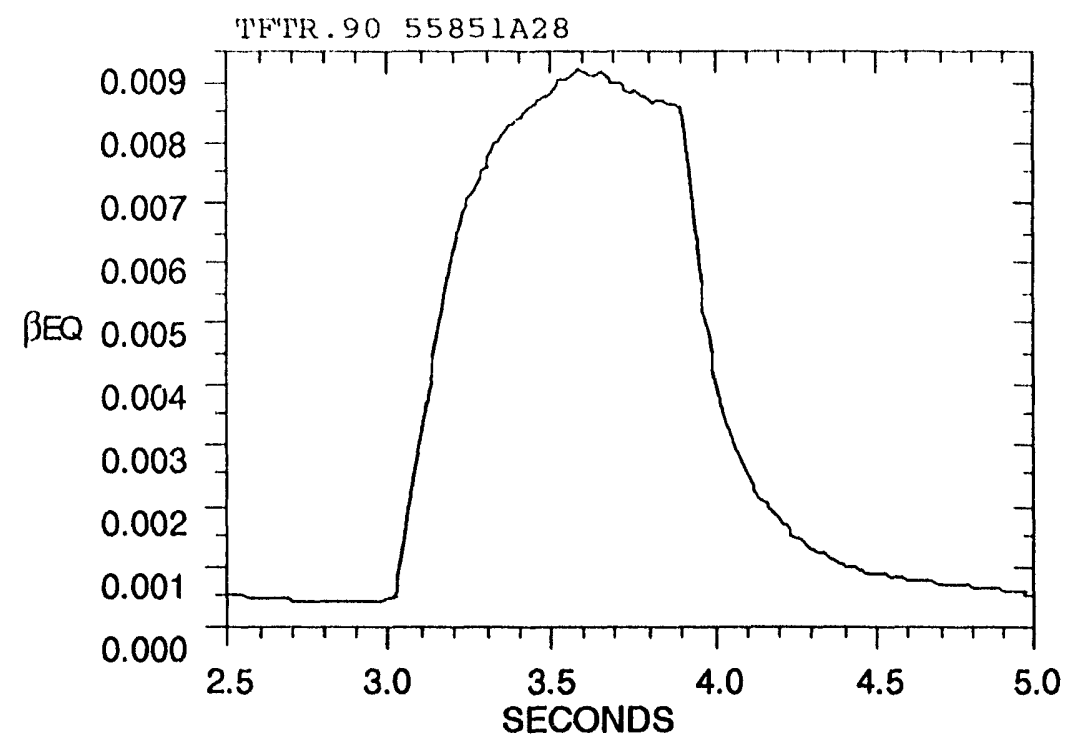

Fig. 5.9 toroidal beta limit vs time.

The beta reaches a maximum of about $0.92 \%$ and sustains the maximum beta much longer than shot \#35782. The beta finally drops when the neutral beams tum off. Shot \#55851 was analyzed for comparison with shot \#35782 since it was clearly different. Although there was some MHD activity during this shot, it does not lead to a beta collapse. 

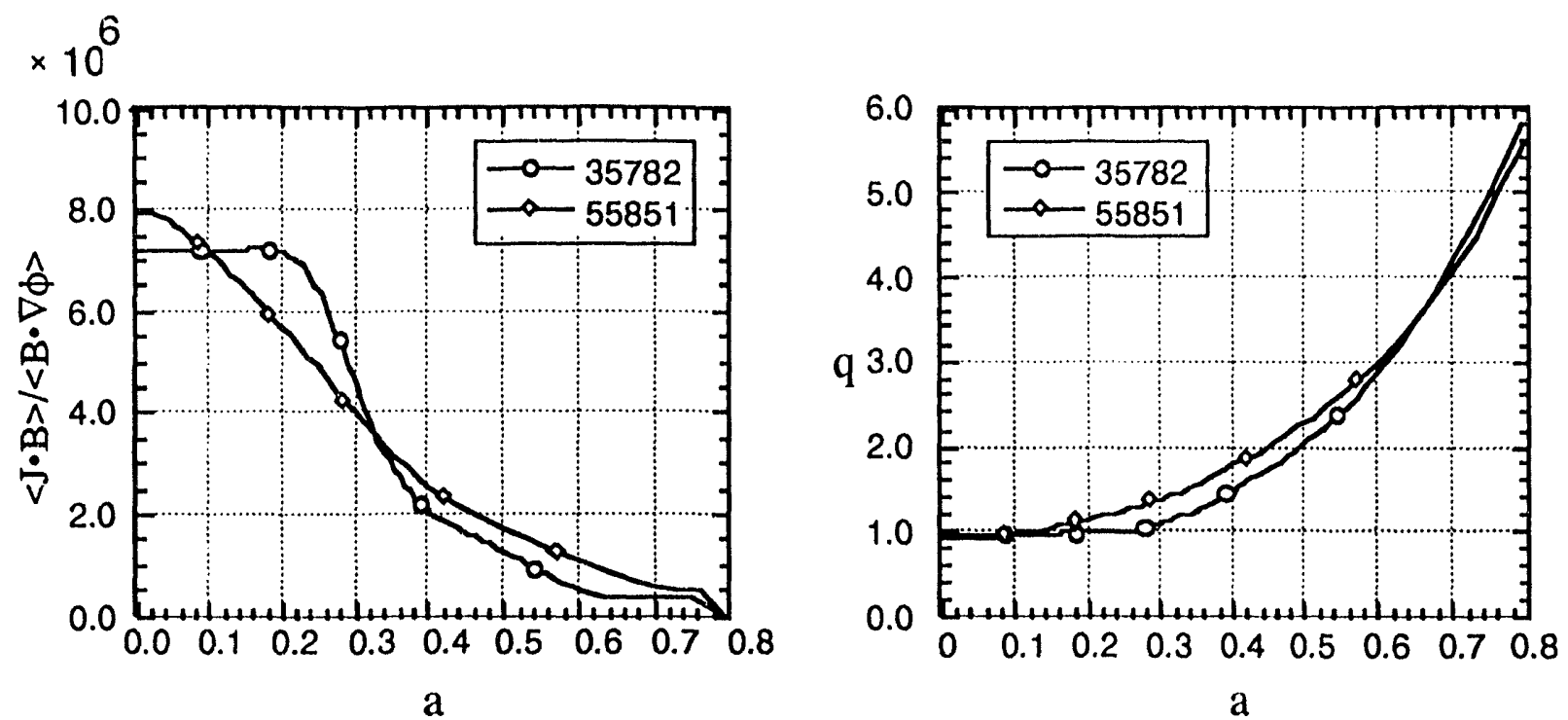

Fig. 5.10 comparison of the $\mathrm{q}$ and $\langle\mathrm{J} \cdot \mathrm{B} / \mathrm{B} \cdot \nabla \phi\rangle$ profiles for the two high beta shots.

Figure 5.10 shows a comparison of the $q$ profile and $\langle\mathrm{J} \cdot \mathrm{B} / \mathrm{B} \cdot \nabla \phi\rangle$ profiles for the two high beta shots. The $\langle\mathrm{J} \cdot \mathrm{B} / \mathrm{B} \cdot \nabla \phi\rangle$ profile for shot $\# 55851$ is more peaked and consequently the q profile is not as flat as shot $\# 35782$. On the basis of this one would expect that this shot would be more stable to infernal modes. The ballooning mode critical pressure gradients are shown in Figure 5.11. In this figure the parameter $\alpha_{\mathrm{T}}$ is plotted versus $\psi$. Here the parameter is defined by

$$
\alpha_{\mathrm{T}}=-\frac{\partial \mathrm{p}}{\partial \psi} \frac{\mathrm{V}(\psi) \Delta \psi}{\mathrm{V}_{0}} \frac{\mathrm{aB}_{0}}{<\mathrm{B}_{\phi}^{2}>\mathrm{I}} \times 10^{8}
$$

where $V(\psi)$ is the volume inside a flux surface, $V_{0}$ is the total plasma volume, $a$ is the minor radius, $B_{0}$ is the toroidal magnetic field at the center of the plasma, $\left\langle B_{\phi}{ }^{2}\right\rangle$ is the volume averaged toroidal field and $I$ is the plasma current. 

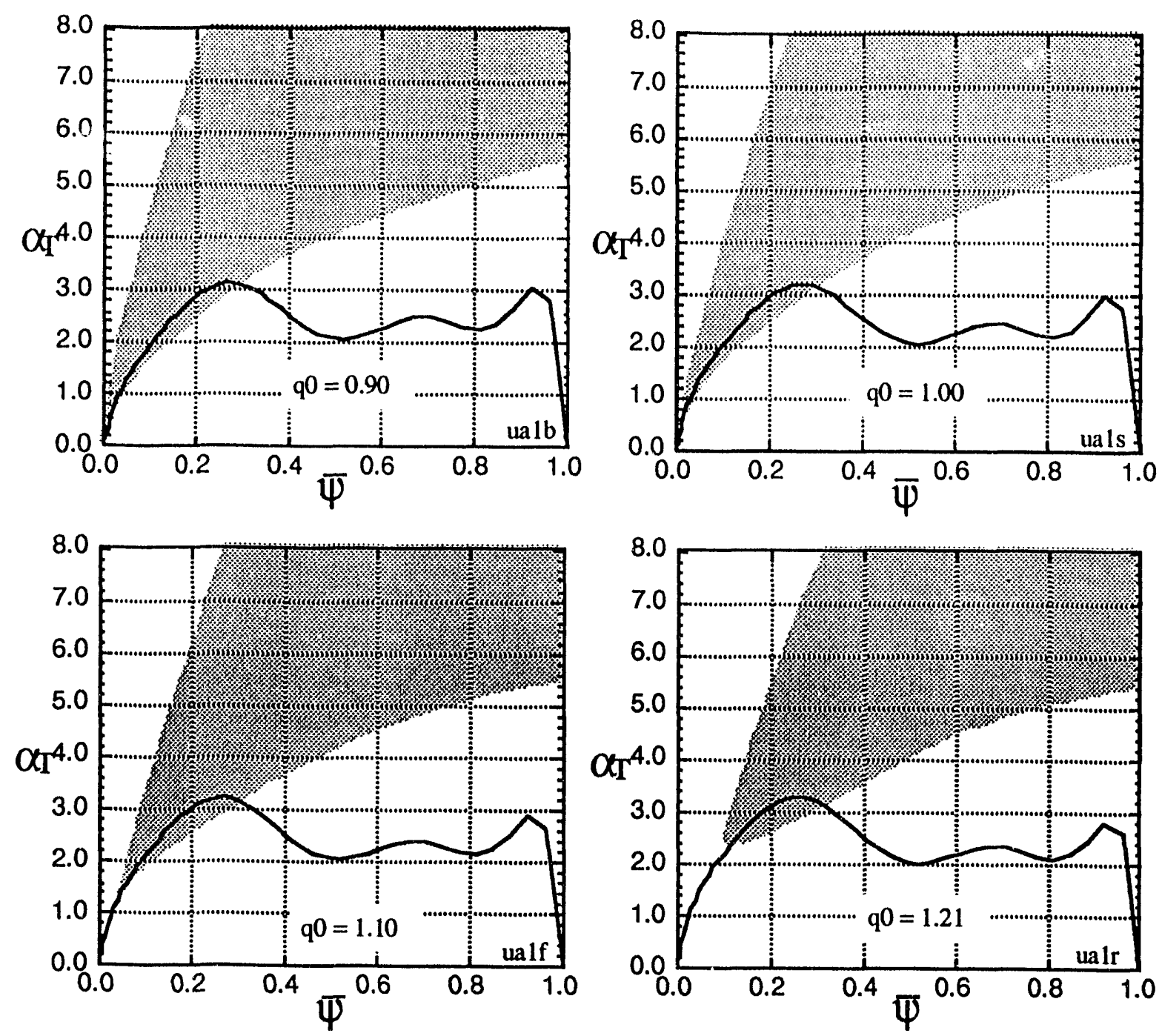

Fig. 5.11 Normalized critical pressure gradient versus normalized $\psi$.

The parameter $\alpha_{T}$ is a useful normalization to the critical pressure gradient. Integrating $\alpha_{T}$ over $\psi$ gives the Troyon beta limit. Hence the normalized beta can be readily obtained from this plot and those modifications to the pressure profile needed to improve the normalized beta can be ascertained. From figure 5.11 it can be seen that the normalized beta is 2.4. The Troyon beta limit has been found to be fairly constant over a wide range of different shaped plasma cross-sections and aspect ratios. Therefore, using $\alpha_{\mathrm{T}}$ allows comparison of the ballooning stability properties of many different plasma cross-sections. In Fig. 5.11 the pressure profile is found to be infinite-n ideal ballooning unstable for the parameters of the supershot. Raising $\mathrm{q}(0)$ by flattening the $\langle\mathrm{J} \cdot \mathrm{B} / \mathrm{B} \cdot \nabla \phi>$ profile in the center does not seem to improve the situation except for a very large $q(0)$. The plots shows that the pressure profile is fairly optimum in the center in the sense that its' shape conforms fairly 
well to the critical $\alpha_{T}$ profile even thou gh it pushes up somewhat into the unst ${ }^{{ }^{\prime}} \cdot$; region. A $q(0) \sim 1.0$ seems to be the most consistent with the experiment. Figure 5.11 suggests that the MHD activity observed in the experiment might be due to low- $n$ ballooning modes. Raising $\mathrm{q}(0)$ higher tends to push the plasma further into the ballooning unstable region. In order to totally stabilize the infinite- $n$ ballooning mode requires $q(0)>1.5$.

Figure 5.12 shows the ideal MHD beta limits as a function and beta and $q(0)$ for shot \#55851. Contrast this figure with Fig. 5.8. Regions of instability for a given $\mathbf{n}$ number are much more spread out. For $\mathrm{q}(0) \sim 1.0$ at a $\beta \sim 0.92 \%$ the $n \geq 4$ modes are found to be unstable. Not shown on the figure are the higher $n$ modes which would also be expected to be unstable from the usual ballooning mode scaling.

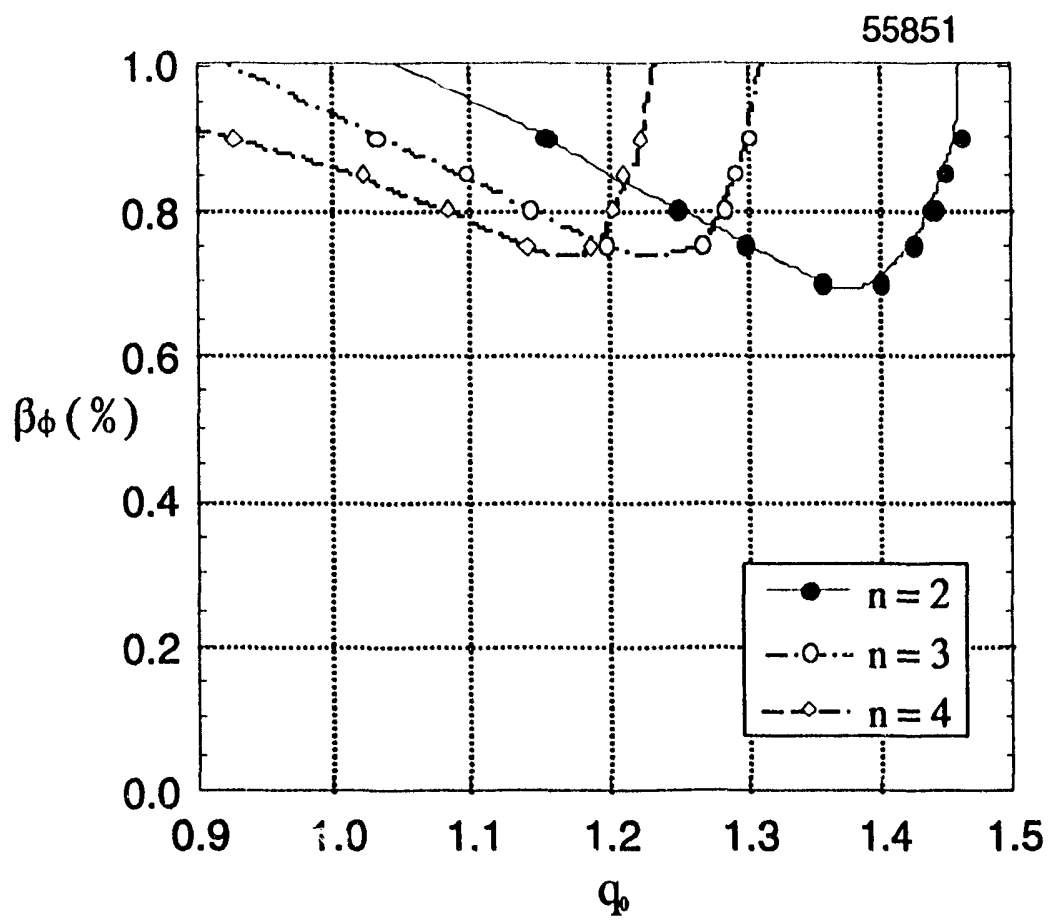

Fig. 5.12 Stability diagram for low $\mathrm{n}$ ballooning modes for shot $\$ 55851$.

From these analyses we can speculate on a scenario that accounts for the observed MHD activity in high beta supershots. The suggestion from these analyses is that if the current profile remains peaked, infernal modes do not occur and the plasma can reach up to the Troyon beta limit. Experiments show that the Troyon beta limit appears to be soft, however, increased MHD activity is observed. This activity and beta saturation is most 
likely due to ballooning modes and/or resistively modified ballooning modes. If for some reason the central current profile gets flattened, the $q$ profile also becomes flat and $q(0)$ rises. This destabilizes the infernal mode. The MHD activity that appears will depend on the trajectory in $\beta, q(0)$ space that the equilibrium follows as time evolves. The first discrete instability region it reaches will tend to be the dominant mode. For regular ballooning modes, high-n modes are predicted to occur (which may not be observable from the outside) which probably lead to profile modifications that tend to make the lower-n modes less unstable. This infernal mode may to be more unstable because of its discrete nature. For it to occur, higher-n modes are not required and hence the profile modifying effects which tend to lead to optimized profiles is absent. The ensuing infernal mode can then lead to a beta collapse.

\subsection{Conclusions}

In this Section, we sought to gain better understanding of the MHD activity seen during high beta supershots. We studied internal modes, resistive infernal modes and tearing modes in high $\beta$ TFTR supershots. Supershots with low central shear were found to be either very close or in a regime unstable to infernal modes. In almost all cases some flattening of the central current profile as given by TRANSP was required. The flattening is modest; e.g. $q(0) \sim 1.3$ is enough to see the $3 / 2$ mode. The dominant mode depends on the trajectory the equilibrium follows in $\beta, q(0)$ space as time evolves. The $2 / 1$ mode requires $q(0)>1.5$ to occur, which may explain why this mode is not often observed. Both infernal mode and ballooning mode instability regions in parameter space are surround by a narrow layer unstable to resistive modes. No flattening of the pressure profile is required to excite this mode. However, because of the narrowness of this layer, it is liliely these modes are simply resistively modified ballooning modes or infernal modes and not tearing modes. While near tearing mode scaling can be found at some point in this layer, it is likely this is just a transition from the pure resistive growth time to the ideal growth time. This is contrary to the conclusions found by Charlton. These studies suggest that the MHD activity observed in supershots is associated with ballooning and/or infernal modes. Supershots can operate at or slightly in the ballooning mode threshold with an increase in MHD activity, but no collapse. However, if the current profile in the center becomes flat a discrete infernal mode occurs leading to a beta collapse. 
[1] L.E. Zakharov,.Nuclear Fusion, 18, (1978), 335

[2] J. W. Connor, H. J. Hastie and J. B. Taylor, Phys. Rev. Lett. 44, (1978), 396.

[3] R. J. Hastie and J. B. Taylor, Nucl. Fusion 21, 187 (1981).

[4] J. Marickam, N. Pomphrey, A. M. M. Todd, Nucl. Fusion 27, (1987), 1461.

[5] M. W. Phillips, M. H. Hughes and A. M. M. Todd, M. Okabayashi,

[6] F. Troyon and R. Gruber, Phys. Rev. Lett. A 110, (1985), 29.

[7] L. A. Charlton, R. J. Hastie and T. C. Hender, Phys. Fluids B 1, 798 (1989).

[8] L. A. Charlton, B. A. Carreras, and V. E. Lynch, Phys. Fluids B 2, 1574 (1990). 
-

0

!

।

-

○

P

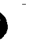

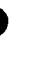




\section{STUDIES OF PKESSURE DRIVEN MODES}

The previous Section introduced the idea that the instabilities responsible for spoiling the confinement in supershots, at least at high $\beta(\sim 1 \%)$, are pressure driven 'infernal' modes or, possibly, small toroidal mode number ballooning modes. In this Section we shall pursue the study of these ideal modes further. In particular we shall gauge the sensitivity of the results discussed previously to both the assumed pressure and current density profiles. It was indicated in Section 3.5 that both the $p$ and $q$ profiles generated by TRANSP are subject to large uncertainties but, nevertheless, provide a useful starting point. Moreover, since this information is obtained in numerical form it is difficult to perform systematic parameter studies, with the exception of altering $q(0)$ as discussed before. Thus, the procedure adopted here involved first fitting the TRANSP profiles to some relatively simple form to facilitate a parameter study. Specifically, we are interested in studying the sensitivity of the results described in the previous Section to changes in both the $\mathrm{p}$ and $\mathrm{q}$ profiles. For the most part, the vehicle used for the ideal MHD studies is the small-n eigenvalue solver, LOBAL, described in Section 3.2, which can accurately locate marginal points. While this code solves a set of reduced MHD equations, the predicted $\beta$ limits were checked using ARES (which solves the full set of MHD equations) and showed satisfactory agreement. Also, ARES was used when actual growth rates were required and, of course, to study the effect of resistivity. However, since the pressure driven modes of interest can excite a large number of harmonics, we were constrained, for reasons concerned with resolution, typically to values of $n \leq 4$ (depending on the qprofile)

\subsection{Sensitivity to the Pressure Profile}

Interestingly, we find that the pressure profiles predicted by TRANSP can be fitted, with sufficient accuracy, by an exponential weighted factor which ensures that both the pressure and its gradient are zero at the boundary. Thus, for example, the pressure profile

$$
p(\psi)=\left(1-\psi^{\alpha_{1}}\right)^{2} \exp \left(-\alpha_{2} \psi\right),
$$

where $\psi$ is the poloidal flux normalized such that $0 \leq \psi \leq 1$, gives a good fit to the TRANSP data from shot $\# 35782$ with $\alpha_{1}=5$ and $\alpha_{2}=3.0$; this is illustrated in Fig. 6.1. Not surprisingly we can then reproduce the results of Section 5 when using the same qprofiles but replacing the numerical pressure profile with this simple analytic form. Here 

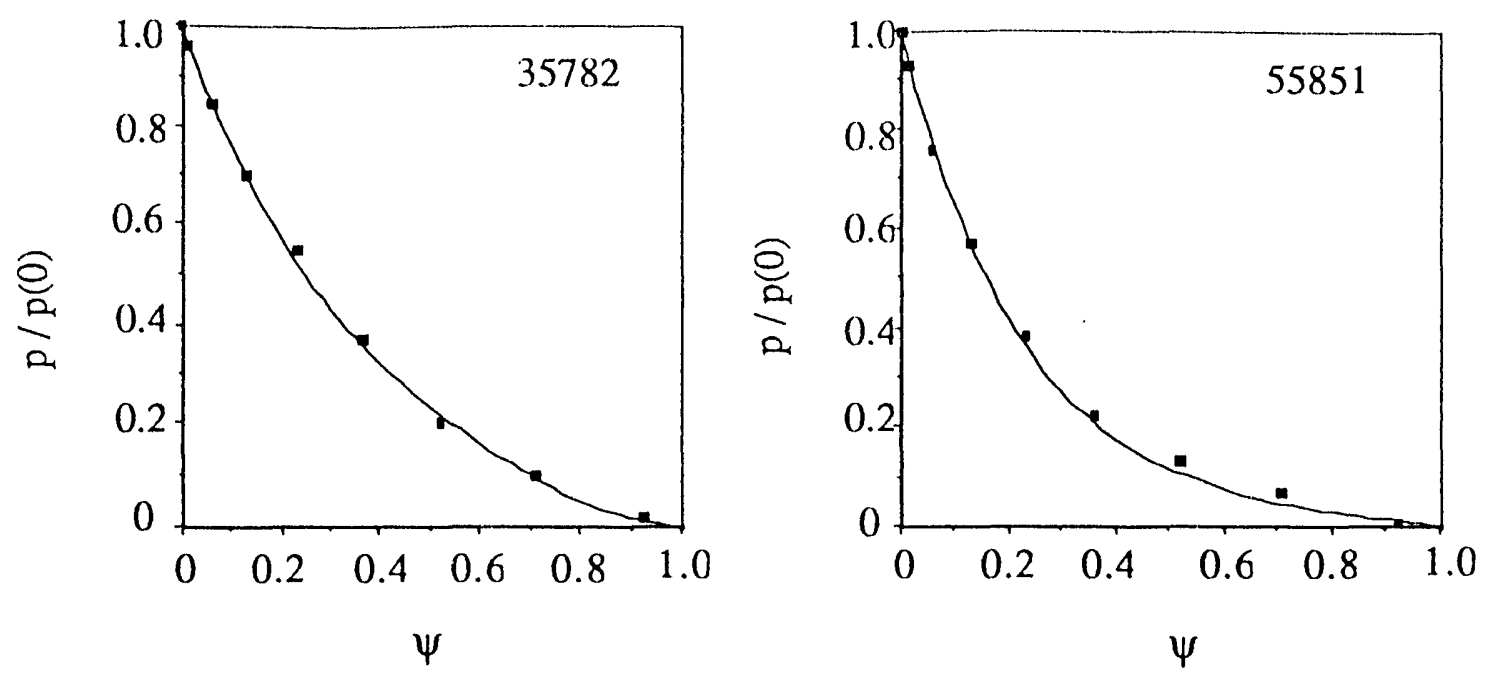

Fig. 6.1 Comparison between analytic pressure profiles and experimental data. The curves illustrate the analytic form while the symbols indicate the experimental data from two high $\beta$ supershots ( $\# 35782$ and 55851 ).

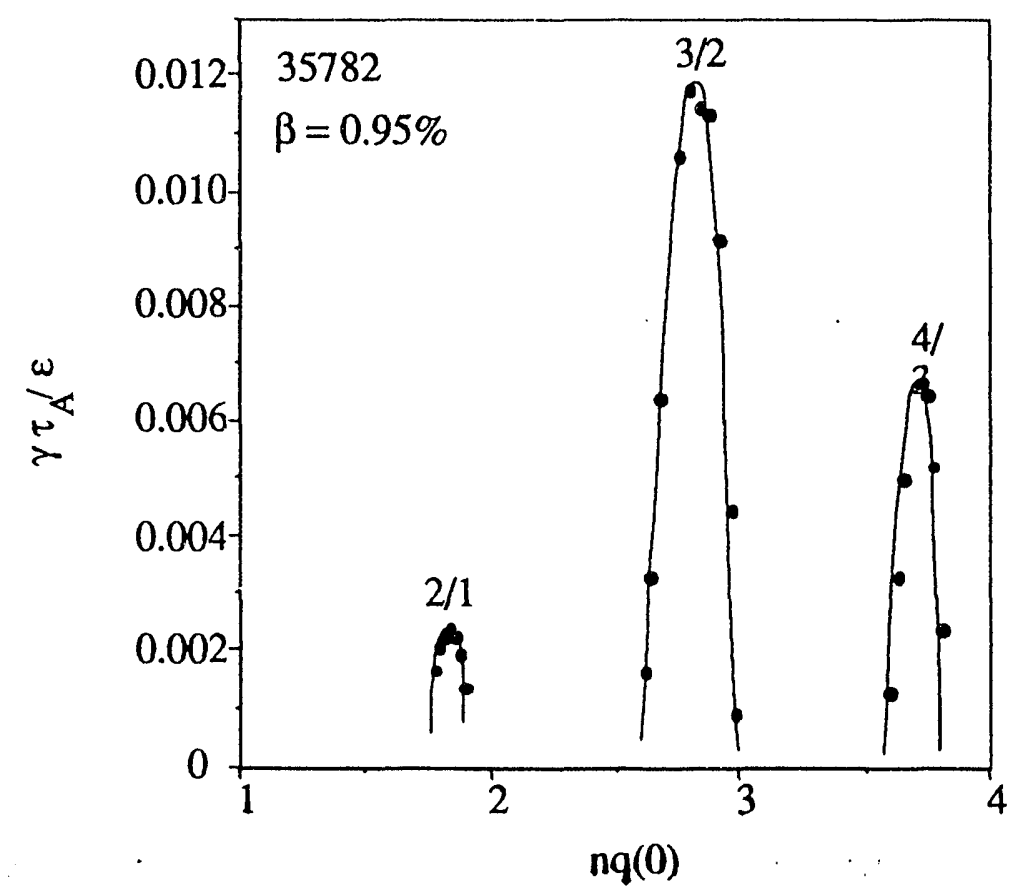

Fig. 6.2 Mode growth rates as $q(0)$ is varied. Bursts of instability appear when $q(0)$ approaches a rational value, which is a characteristic feature of the infernal modes. 

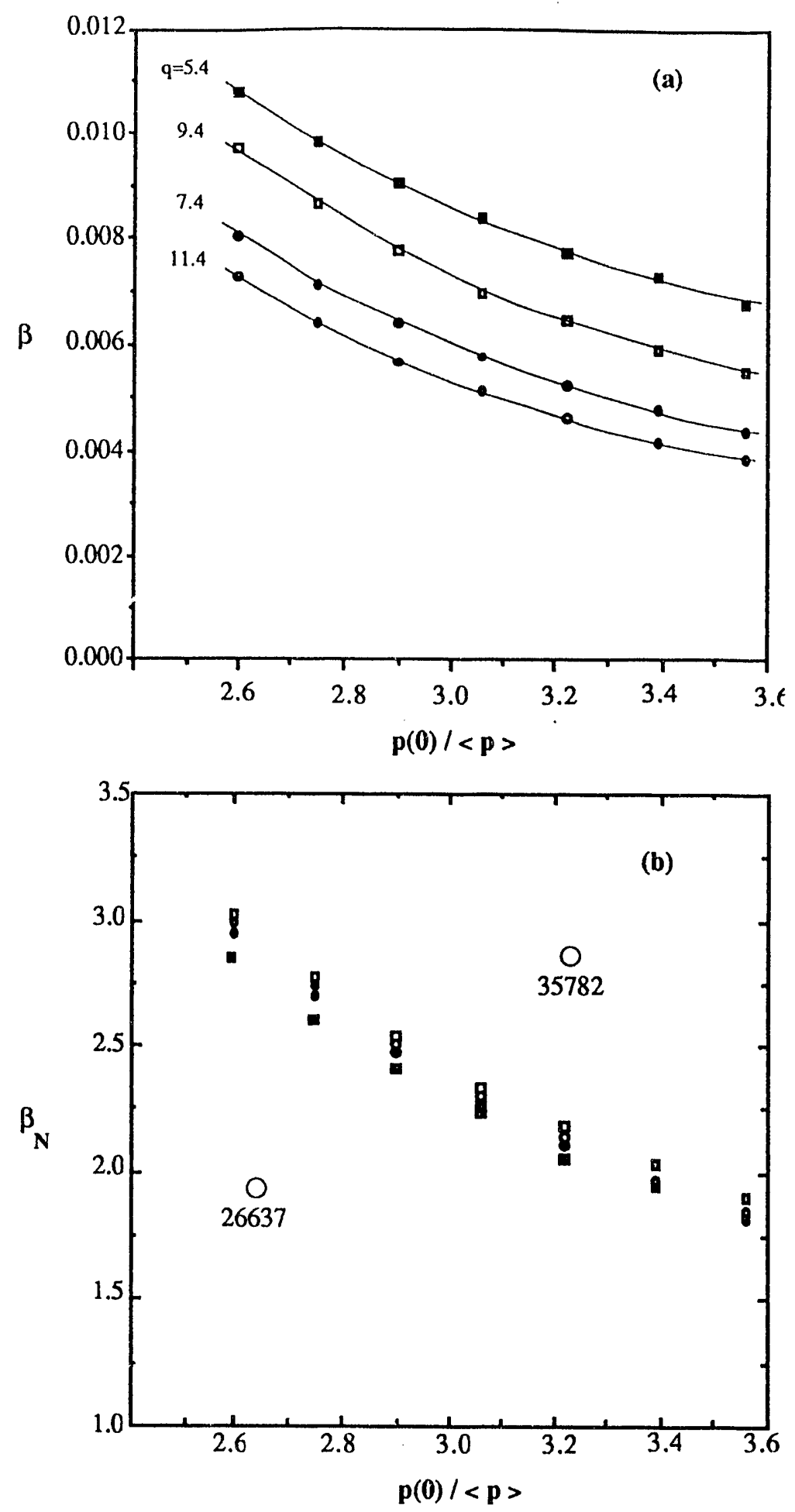

Fig. 6.3 Marginally stable $\beta$ for the $3 / 2$ infernal mode as function of the peakedness of the pressure profile. In each case, $q(0)=1.44$.

Diagram (a) shows the limiting $\beta$ with $q$ (edge) as a parameter. Diagram (b) plots the normalized values $\beta_{\mathrm{N}}=\beta /(\mathrm{I} / \mathrm{aB})$. For reference Fig. (b) shows the nominal locations of the supershots \#35782 and \#26637. 
we shall discuss the effect of altering the pressure gradient inside the rational surfaces of interest. A similar study of systematically altering the q-profile is deferred to Section 6.2.

It was shown in the previous Section that small-n infernal instabilities can appear when the shear is small and $\mathrm{q}(0)$ is elevated, even though the equilibria are stable to ballooning modes. Thus, keeping the pressure and its distribution fixed but altering $q$ in the manner described in Section 3.4, we obtain the result illustrated in Fig. 6.2. Referring to this diagram, it is evident that a burst of instability appears when $q(0)$ is close to a rational value.

We then select one of these modes (specifically the $3 / 2$ instability when $q(0)=1.44$ ) and examine the effect of varying the pressure gradient inside the rational surface by changing the exponent $\alpha_{2}$ in our formula for the pressure. Using LOBAL we find the marginally stable values of $\beta$ for each $\alpha_{2}$. The result is shown in Fig. 6.3 (a) where we plot the marginal points as a function of the peakedness of the pressure profile, $p(0) /<p>$, (where $\langle\mathrm{p}\rangle=\int_{0}^{1} \mathrm{~d} \psi \mathrm{p}(\psi)$ ) with $\mathrm{q}($ edge) or, equivalently, the total current as a parameter but keeping $q(0)$ fixed at 1.44. Replacing $\beta$ by the normalized values $\beta_{N}=\beta /(\mathrm{L} / \mathrm{aB})$, we obtain the result shown in Fig. 6.3(b). This figure shows that the limiting beta determined by the infernal mode follows a Troyon scaling $\beta_{N}=C_{T}$ but that the value of $C_{T}$ is sensitively dependent on the peakedness of the pressure profile. It is evident that this $3 / 2$ infernal mode can impose a severe limit on $\beta$. For reference, we indicate the nominal locations of the high $\beta(-1 \%)$ supershot \#35782 considered in Section 5 , together with the low $\beta(-0.4 \%)$ shot \#26637, whose resistive stability was studied in Section 4 . Both shots were observed experimentally to be $3 / 2$ unstable.

Referring to Fig. 6.3(b), it is evident that shot \#35782 is predicted by ideal MHD theory to be highly unstable to the $3 / 2$ infernal mode when $q(0)=1.44$, the value where the instability is most virulent. Note that, if $q(0)$ were reduced, the limiting $\beta_{N}$ would increase and the location of shot \#35782 would be closer to marginal stability. On the other hand, shot \#26637 lies well into the stable region. We recall, however, that approximately half the shots comprising the 266-series were, indeed, stable. It is possible that the unstable plasmas of this series had pressure profile substantially more peaked than that predicted by TRANSP. 'This information on individual shots is lost in the manner in which the data is collated. 


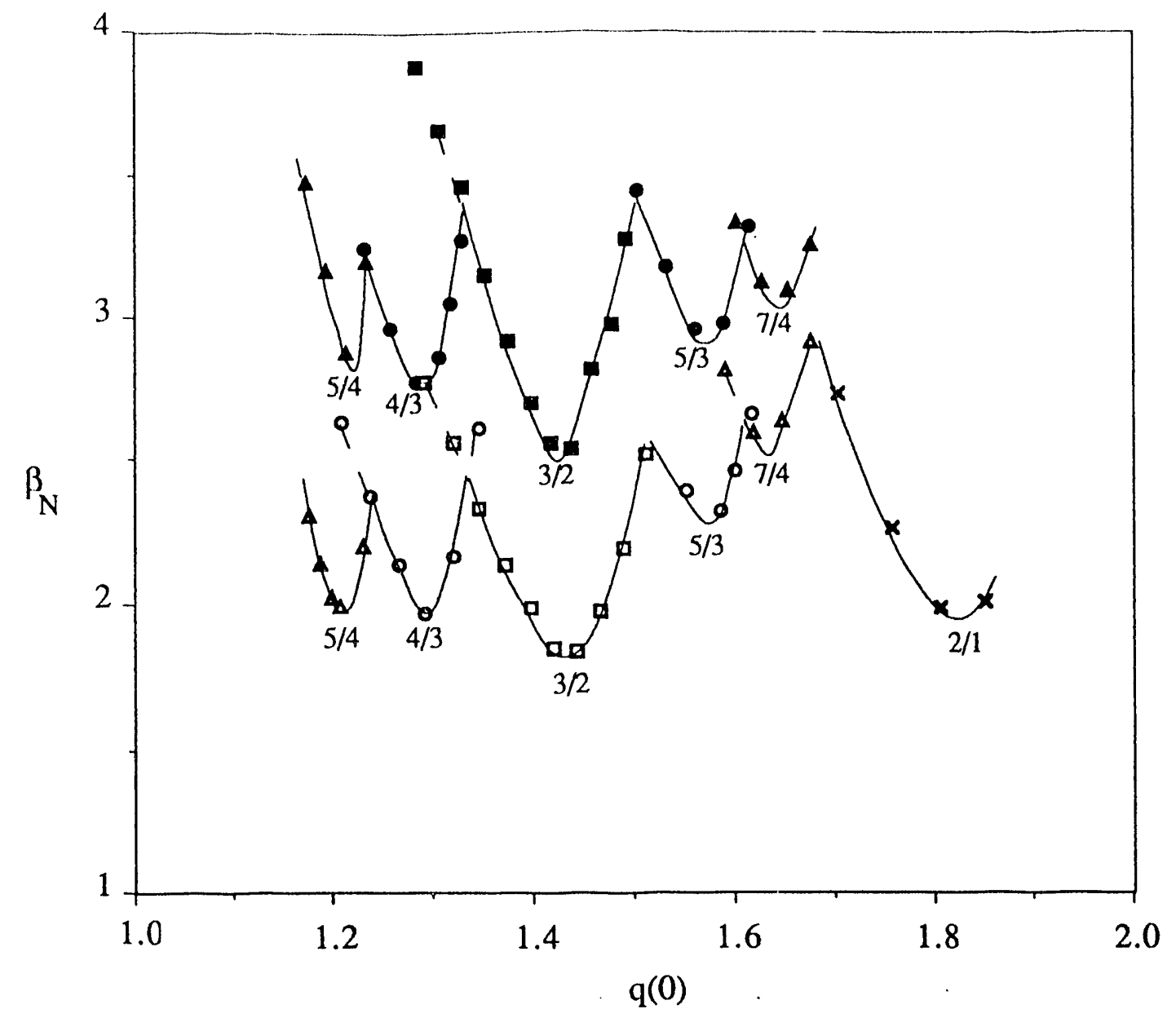

Fig. 6.4 Stability diagram showing the limiting $\beta_{\mathrm{N}}$ as a function of $q(0)$. The lower graph corresponds to a highly peaked pressure profile with $\alpha_{2}=3.2$; the upper curve has $\alpha_{2}=2.2$.

Fig. 6.4 plots the limiting values of $\beta_{\mathrm{N}}$ as a function of $q(0)$ for two peakedness factors $\left(\alpha_{2}=2.2\right.$ and $\left.\alpha_{2}=3.2\right)$ corresponding to the range of TRANSP profiles that we have studied. This diagram also highlights the severe limitation on $\beta$ when the pressure profile is highly peaked. The mode structure that will be excited depends on the trajectory followed in this diagram. Thus, if $q(0)$ increases during the beam heating phase, as suggested by the fact that the $1 / 1$ sawtooth mode vanishes, then we would expect that the higher $\mathrm{m} / \mathrm{n}$ modes appear first. We also note that the $2 / 1$ instability appears only in the most highly peaked pressure profiles studied here. The reason for this behavior is not presently understood, but we note that the $2 / 1$ mode is observed only very infrequently in the experiments( $\sim 2 \%$ of shots). 


\subsection{Sensitivity to the Current Density Profile}

Examination of the q-profiles generated by TRANSP shows that these too can often be approximated with sufficient accuracy by a simple analytic form. For example, the profile

$$
q(\psi)=q(0)\left(1+C \psi^{\alpha_{3}}\right) \exp \left(\alpha_{4} \psi\right)
$$

is compared in Fig. 6.5 with the data obtained from TRANSP for shot \#35782 (but with $q(0)=1.44)$ using $\alpha_{3}=4, \alpha_{4}=0.13, C=2.3$, and $q(0)=1.44$. Since $(d q / d \psi) / q \approx \alpha_{4}$ over a predictable fraction of the radius, we can interpret $\alpha_{4}$ as the shear inside the rational surface. Again, we have shown that this analytic form for $q$ yields results consistent with those obtained previously using the numerical data from TRANSP.

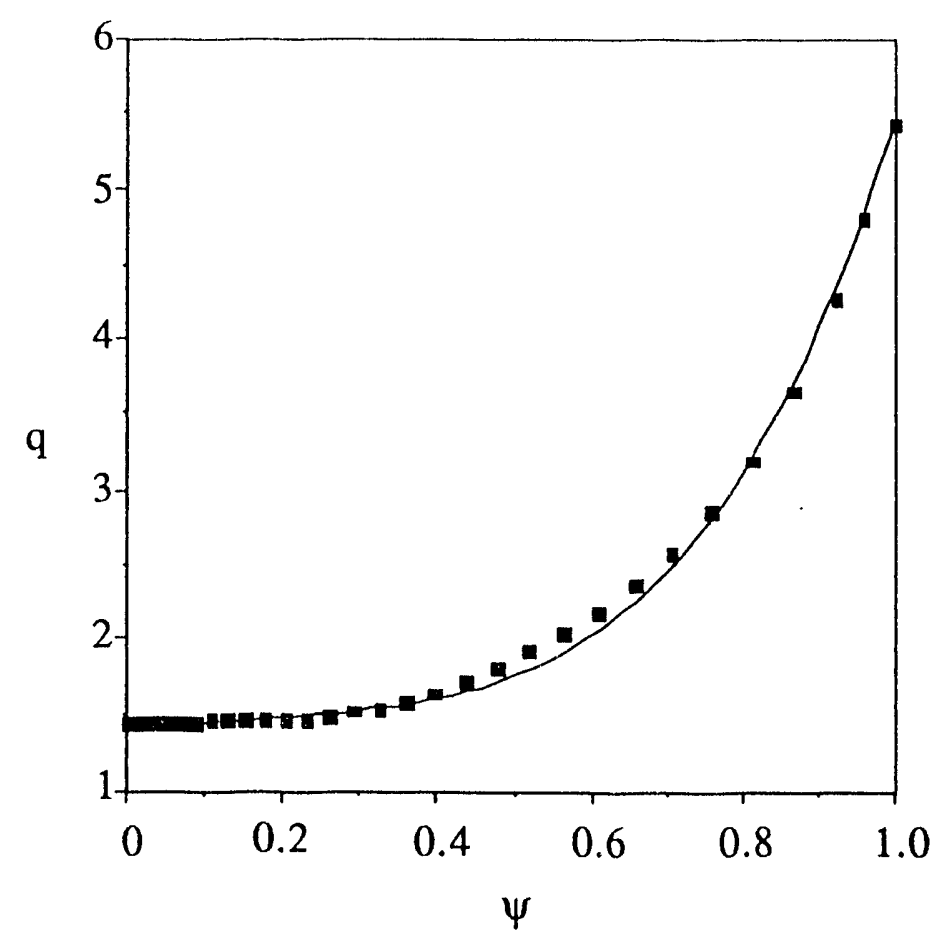

Fig. 6.5 Comparison of the TRANSP q profile with an analytic formula. The analytic curve is obtained from $q(\psi)=q(0)\left(1+C \psi^{\alpha} \alpha_{3}\right)$ $\exp \left(\alpha_{4} \psi\right)$ with $q(0)=1.44, C=2.3, \alpha_{3}=4$ and $\alpha_{4}=0.13$. This is compared with data (solid symbols) from TRANSP run for shot \#35782 with $q(0)$ at 1.44

Using the above formula for $\mathrm{q}$ and fixed $\mathrm{q}(0)=1.44$ we systematically make the current density profile more peaked by increasing $\alpha_{4}$ while adjusting $C$ to keep the edge 


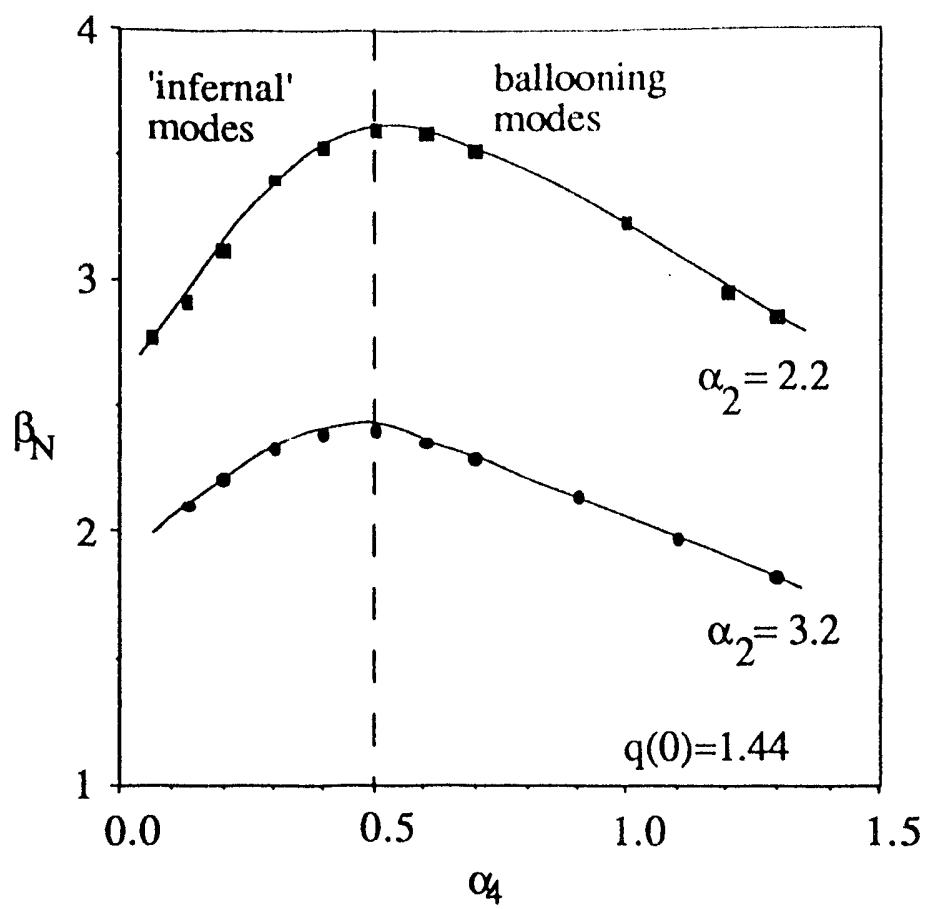

Fig. 6.6 The limiting $\beta_{N}$ for ideal, pressure driven modes as a function of the shear. When $\alpha_{4}$, the shear, is small $(<0.5) \beta$ is limited by the infernal mode. At large values of $\alpha_{4}$ the $\beta$ limit is determined by ballooning modes. The parameter $\alpha_{2}$ measures the peakedness of the pressure profile. In either case, highly peaked pressure profiles severely limit the maximum attainable $\beta$.

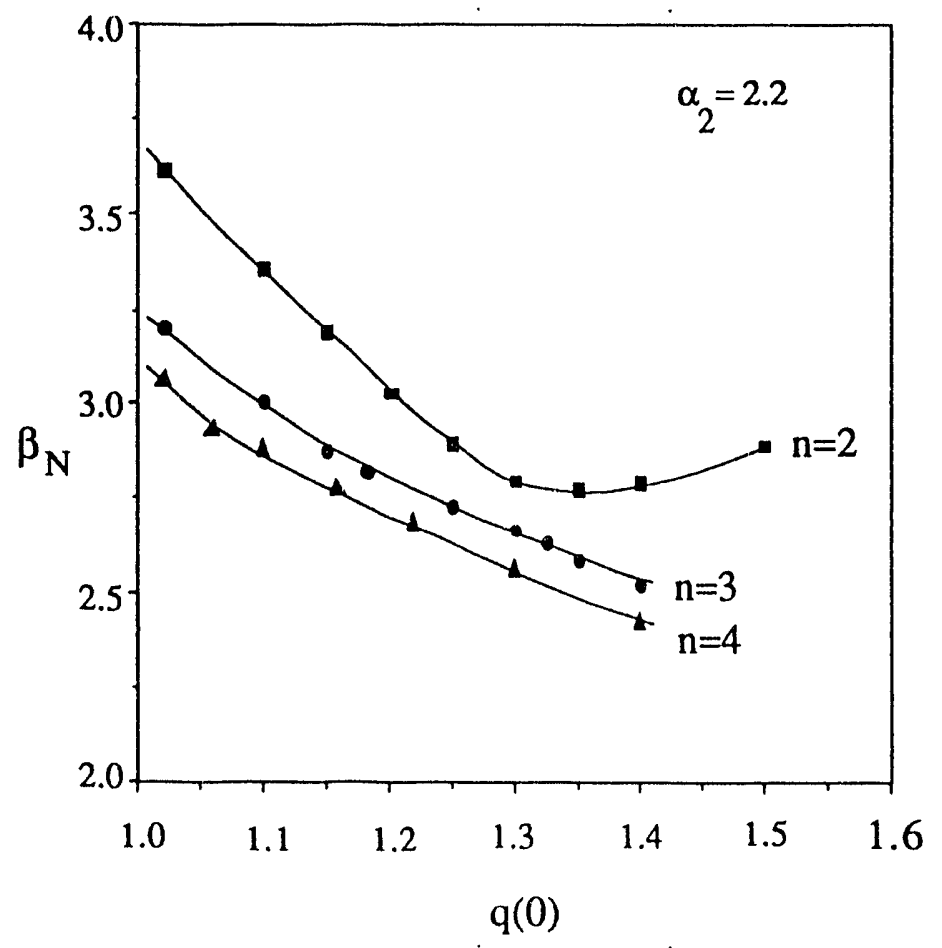

Fig. 6.7 The limiting $\beta$ as a function of $q(0)$ for ballooning modes. This graph corresponds to a relatively broad pressure distribution with $a 2=2.2$. In the range of parameters indicated we do not find a $n=1$ mode. 
value of q constant. This makes the q-profile steeper and increases the shear. Fig. 6.6 shows how the normalized limiting beta, $\beta_{\mathrm{N}}$, then changes with $\alpha_{4}$. When $\alpha_{4}$ is small (or, equivalently, the shear is small) we find the infernal mode already discussed. As $\alpha_{4}$ is increased, the limiting $\beta_{\mathrm{N}}$ also increases reaching a maximum when $\alpha_{4} \sim 0.5$. Subsequently, as $\alpha_{4}$ continues to increase the limiting $\beta_{N}$ decreases. In this latter region, where the shear is large, the instability which appears when the limiting $\beta_{N}$ is exceeded is not an infernai mode but is an $n=2$ (in this example) ballooning mode. In either case, the maximum attainable $\beta$ is severely limited when the pressure profile is highly peaked. For reference, the value of $\alpha_{2}$ which best fits the pressure data from shot \#35782 is $\sim 3$.

The two modes of instability, which we have referred to as ballooning and infernal, have quite different characteristics. For example, as $q(0)$ is varied an infernal mode with a particular structure appears only in a narrow band of $q(0)$. On the other hand, the ballooning mode with a given toroidal mode number persists over a wide range of $q(0)$ including $q(0)=1$. This is shown in Fig. 6.7 where the limiting $\beta_{N}$ for ballooning is plotted against $\mathrm{q}(0)$; this should be compared with the corresponding diagram, Fig. 6.4, for the infernal modes. It is also apparent from Fig. 6.7 that in this case of the ballooning instabilities, that the larger-n modes are the most unstable as predicted by analytic theory. In either case, the highly peaked pressure profiles, which are a characteristic feature of supershots and (evidently) responsible for their generally good confinement properties, can ultimately impose a severe limit on the maximum attainable $\beta$.

\subsection{Conclusions}

These studies of pressure driven ideal MHD instabilities have attempted to gauge the sensitivity of the stability calculations to changes in both the pressure and current density profiles used to construct supershot equilibria. The conclusions are as follows:

- Ideal MHD theory predicts that high $\beta$ supershot plasmas are unstable to either low- $n$ ballooning modes or infernal modes, depending on the peakedness of the current density profile. At low $\beta$ the comparison between theory and experiment is less convincing unless the pressure profiles in those plasmas observed to be unstable are substantially more peaked than the is predicted by TRANSP. 
- $\quad$ The limiting $\beta$ for stability to the infernal mode follows a Troyon like scaling, $\beta=\mathrm{C}_{\mathrm{T}}(\mathrm{I} / \mathrm{aB})$, but the value of $\mathrm{C}_{\mathrm{T}}$ depends sensitively on the peakedness of the pressure profile. The infernal mode can severely limit the maximum attainable $\beta$ especially for the more highly peaked pressure profiles.

- A significant qualitative feature of the infernal mode is that a given structure, $\mathrm{m} / \mathrm{n}$, is excited only when $\mathrm{q}(0)$ is close to that rational value. Thus if $q(0)$ increases during the beam heating phase of the supershot then the higher $n$ numbers will appear first.

- For reasons not yet understood, the $2 / 1$ infernal mode is excited only when the pressure profile is very highly peaked. This, together with the highly elevated values of $q(0)(>1.7)$ required to excite the mode, could be a reason for the relatively infrequent appearance of the $2 / 1$ instability in the experiments ( about $2 \%$ of all plasmas).

- If the shear is substantially larger than is predicted by TRANSP the unstable modes are ordinary low- $n$ ballooning modes. These instabilities do not rely on elevated values of $q(0)$, and a mode with a given toroidal mode number can be excited over a wide range including $q(0)=1$. In this case the higher- $n$ numbers are always the most unstable, in accordance with analytic theory. 
•

-

$\bullet$

|

।

-

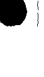

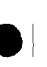




\section{STUDIES OF THE 1/1 MODE IN TOKAMAKS}

This Section summarizes exploratory calculations, carried out in collaboration with W. Stodiek at PPPL, which attempt to clarify the role of the $1 / 1$ mode in tokamaks. Thus, although the sawtooth oscillations in an ohmically heated plasma have been observed, documented and discussed since the earliest days of tokamak research, the phenomenon still attracts considerable attention and is still very poorly understood. More recent experimental observations, first reported by the TEXTOR group [1] and subsequently confirmed by several other tokamak experiments $[2,3]$, that the central value of the safety factor, $\mathrm{q}(0)$, remains substantially less than unity $(\sim 0.6-0.7)$ throughout the entire sawtooth cycle have exacerbated the situation. Here we address only the marginal stability properties of the $1 / 1$ internal mode though not the accompanying sawtooth process itself. In particular, using ARES and the PEST III code (which measures $\Delta^{\prime}$ ) we have examined both the ideal and resistive stability of equilibria where $q<1$ over some substantial fraction of the plasma radius. We have studied the effects of varying the aspect ratio of the $q=1$ surface, the current density profile, the Lundquist number and the fluid compressibility.

Initial equilibrium pressure and current density profiles for this study were obtained from the TEXTOR tokamak which are well documented and for which we have ready access to the experimental information. We have considered two situations: (a) plasmas with qedge $>3$ where the current density profiles, when appropriately normalized, develop into a universal shape, independent of the major and minor radii, the toroidal field and the total current; (b) an 'extreme' case with qedge $\sim 2$ where the current profile develops a substantially different shape with a well resolved shoulder inside $\mathrm{q}=1$, resulting in much reduced shear at the rational surface. Both profiles are illustrated in Fig. 7.1 where we plot the toroidal current density as a function of the major radius across the midplane and, in each case, $\mathrm{q}(0) \sim 0.7$.

Since the shear at the $\mathrm{q}=1$ surface is a parameter that we wish to alter and this, in turn, depends on the width, $\omega$, of the shoulder in the current density profile which may develop within the rational surface, we have devised an algorithm such that equilibria can be generated with varying $\omega$. As $\omega$ is altered, $q(0)$ changes appropriately, increasing toward unity as the shoulder fills the region between the rational surface and the axis corresponding to 'complete reconnection'. These equilibria were subsequently analyzed 

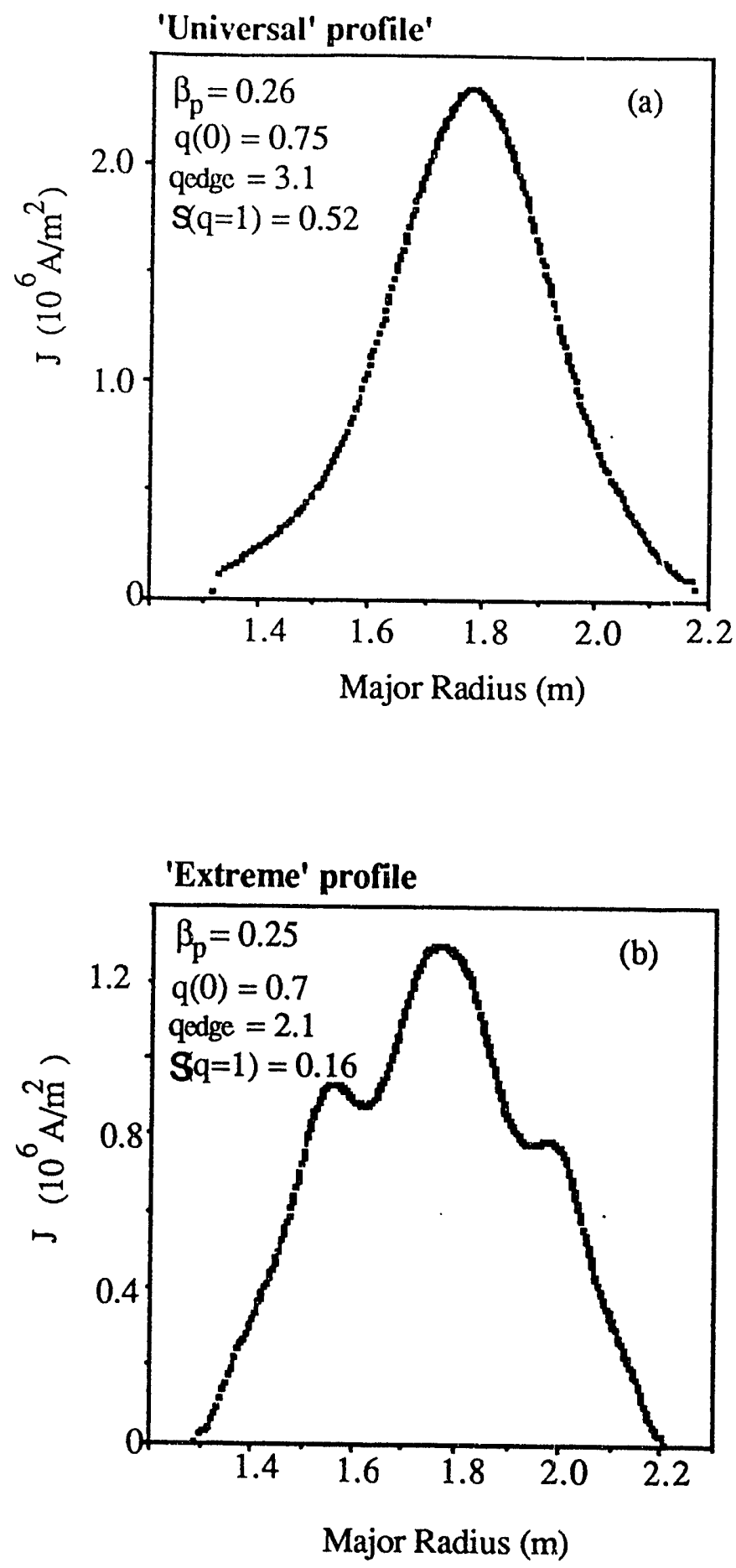

Fig. 7.1 Current density distributions in the TEXTOR tokamak

Diagram (a) illustrates the 'universal' profile when $q>3$. Graph (b) illustrates the 'extreme' case where q 2. A well resolved shoulder appears inside the $q=1$ surface in this latter case. 


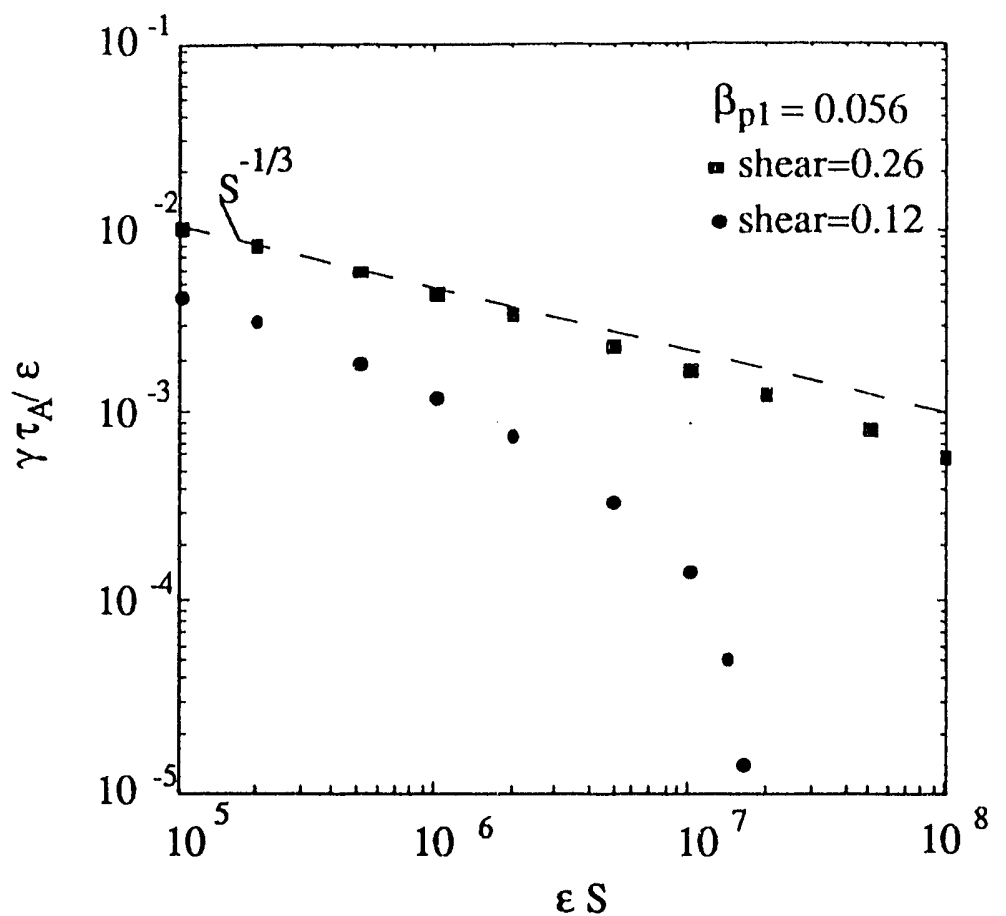

Fig. 7.2 Resistive mode growth rates as functions of the Lundquist number.

for both ideal and resistive stability using ARES. Our initial studies of these TEXTOR plasmas employed the version of ARES which imposed the constraint of incompressible perturbations, which, as indicated later, has interesting consequences.

It is well known from analytic theory [4] that the ideal internal kink is stable in toroidal geometry, even when $\mathrm{q}<1$, provided that the relevant parameter $\beta_{\mathrm{pl}} \equiv \frac{8 \pi}{\mathrm{I}^{2}\left(\mathrm{r}_{\mathrm{S}}\right)} \int_{0}^{\mathrm{r}_{\mathrm{S}}} \mathrm{r}^{2}\left(-\frac{\mathrm{dp}}{\mathrm{dr}}\right) \mathrm{dr}$, which is a measure of the poloidal field energy within $\mathrm{q}=1$, is sufficiently small. Indeed, we find that Bussac's suggestion that $\beta_{\mathrm{p} 1}<0.2$ for ideal MHD stability is an useful 'rule of thumb', though the precise value of $\beta_{\mathrm{p} 1}$ for stability is sensitively dependent on the shear at $\mathrm{q}=1$ when this is small (but, in our experience does not exceed 0.2). Both the 'universal' and 'extreme' experimental profiles considered here were found to be close to marginal stability to the ideal internal kink.

Considering the extreme profile with qedge $\sim 2$, Fig. 7.2 shows the behavior of the resistive modes which are found when $\beta_{\mathrm{p} 1}$ is substantially smaller than the critical value for ideal instability. Referring to Fig. 7.2 , when the shear at $q=1$ is moderately large $(0.26$ in the example shown), a resistive mode is found whose growth rate scales as $S^{-1 / 3}$ (often referred to as a resistive kink) at low $S$, but tending to the tearing mode scaling $S^{-3 / 5}$ when $S$ is sufficiently large. For the aspect ratio considered here (6.9 measured at $q=1)$ the 
transition occurs when $\varepsilon S>10^{8}$. For reference, the value of $\varepsilon S$ relevant to the TEXTOR plasmas considered is typically $2-5 \times 10^{7}$. Fig. 7.2 also shows that when the shear is reduced (from 0.26 to 0.12 ) the tearing branch vanishes and the resistive kink mode tends rapidly towards stability within the $S$-range of interest. Thus, with small enough shear and sufficiently large $S$ we find a regime stable to both ideal and resistive modes.

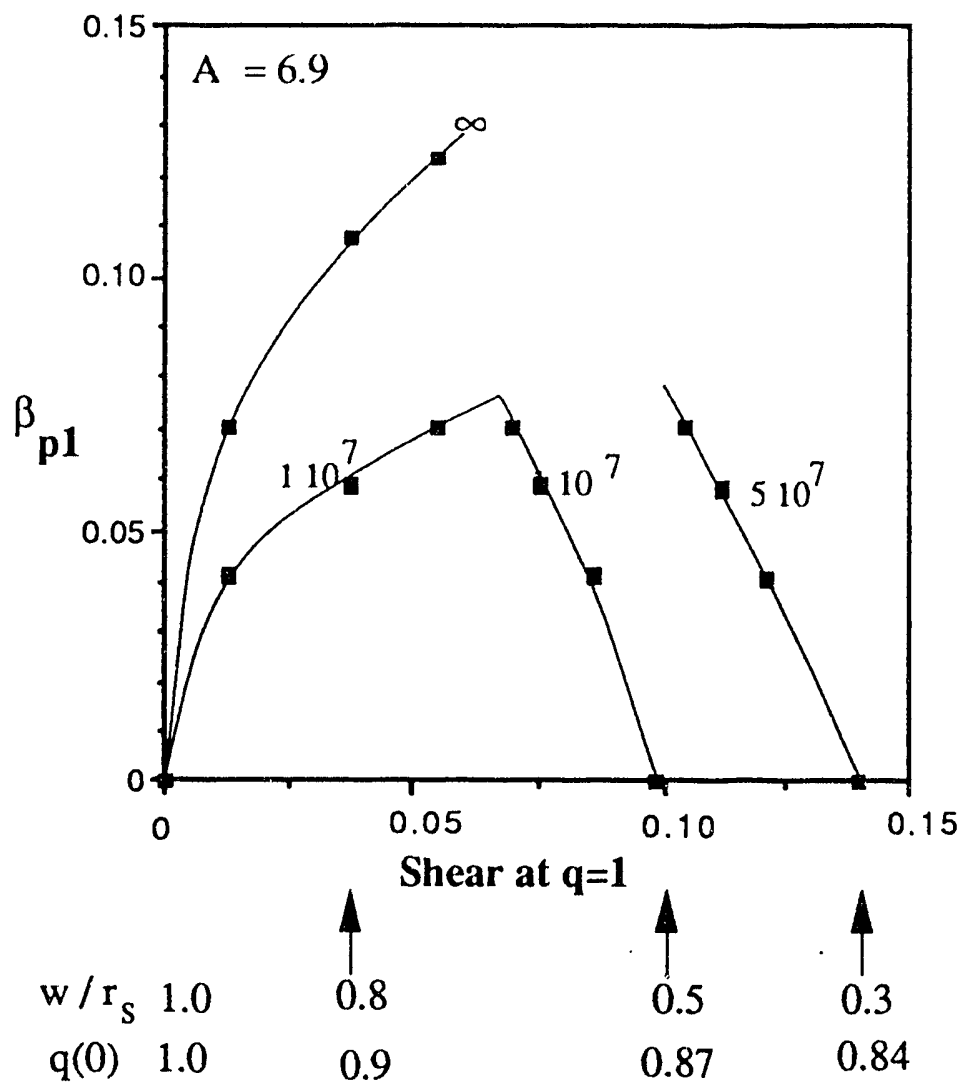

Fig. 7.3 Contours of marginal stability for $1 / 1$ modes with different values of $\varepsilon S$.

The shear is altered by changing the width of the shoulder in the current density profile. This width is indicated as a fraction of the $q=1$ radius, $r_{S}$, which is held fixed. The corresponding values of $q(0)$ are also indicated.

Fig. 7.3 shows the contours of marginal stability for the $m=1$ ideal and resistive modes in the ( $\beta_{\mathrm{p} 1}$, shear) plane, again for the extreme current profile. This is an interesting diagram since a closed trajectory can be envisaged corresponding to a sawtooth cycle. Thus, our original picture was that the highly localized resistive mode would, through enhanced transport, reduce the shear at $q=1$ until the (right hand) marginal boundary is 


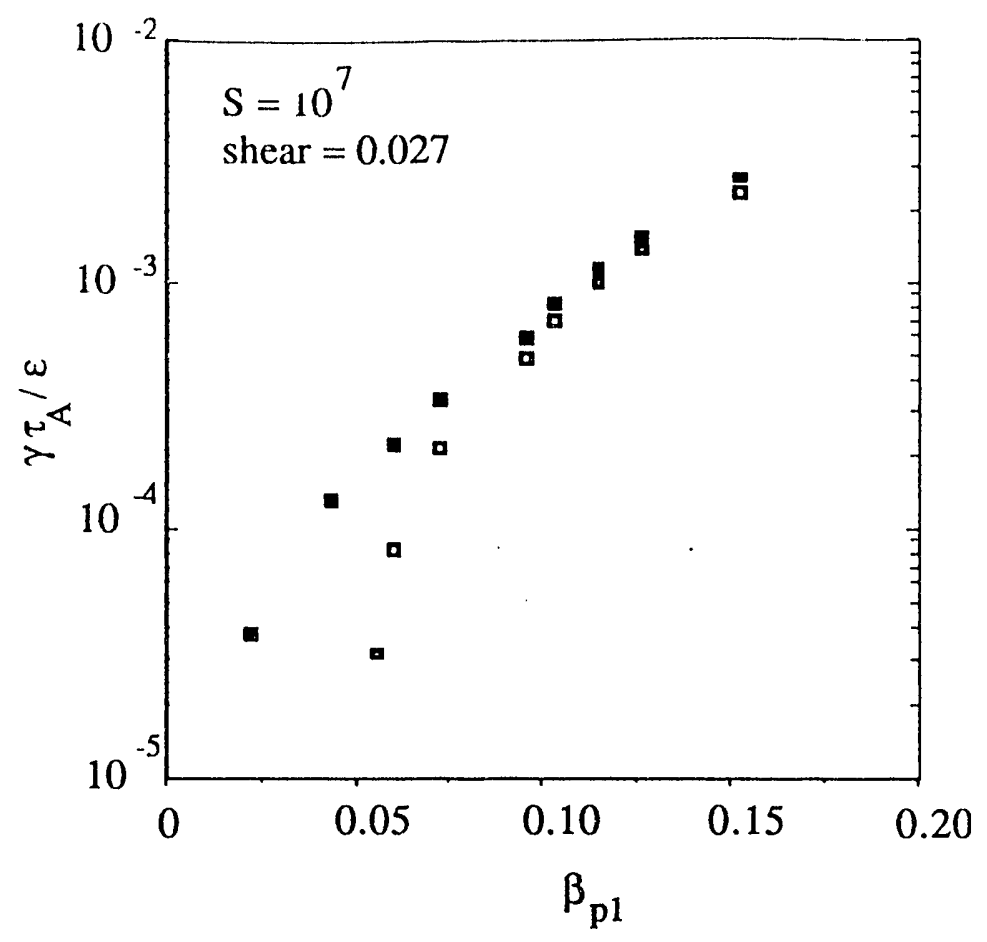

Fig. 7.4 Variation of compressible and incompressible mode growth rates with $\beta \mathrm{pl}$ at fixed shear.

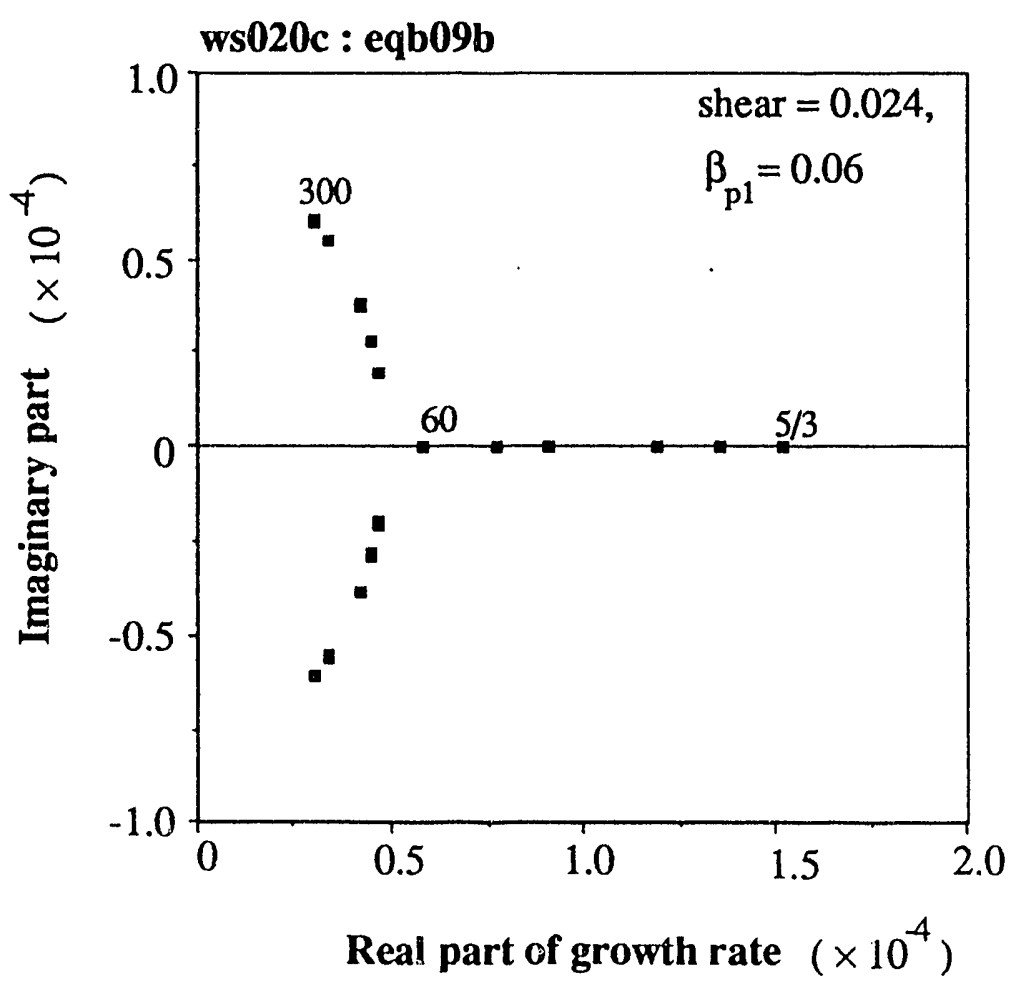

Fig. 7.5 Real and imaginary parts of the growth rate as the adiabatic index is increased 
reached. Heating would cause $\beta_{p} 1$ to increase along this boundary up to the apex. Any further change would trigger the rapidly growing ideal mode, corresponding to the sawtooth crash, which is subsequently stabilized as the pressure gradient within $q=1$ is relieved and the cycle can be repeated.

However, the initial excitement generated by the results discussed above was short lived as a version of ARES with compressible perturbations became available! Thus, when this exercise is repeated with finite fluid compressibility, the stable region illustrated in Fig. 7.3 essentially vanishes. That is, the entire area stable to the ideal $1 / 1$ internal kink is always unstable to resistive modes (except possibly at zero or very small pressure). This is one example where the constraint of incompressibility can evidently lead to erroneous conclusions. For example, Fig. 7.4 compares the resistive mode growth rates, at fixed shear, as $\beta_{\mathrm{p} 1}$ is altered. In the incompressible limit, the growth rate tends rapidly towards zero at finite pressure; in the compressible case, however, the mode persists. Fig. 7.5 shows how this unstable mode behaves when the adiabatic index, $\Gamma$, is increased from its initial (and usual) value of $5 / 3$. Initially the mode is purely growing, but as $\Gamma$ increases, the growth rate decreases, eventually becoming complex (oscillatory growth) with the real part tending toward zero at large enough $\Gamma$, in accordance with the result found with strict incompressibility.

To recapitulate, we find equilibrium conditions stable to resistive modes only with the (artificial) constraint of incompressibility. Even in this case, however, both the critical shear and the critical pressure gradients that are computed are embarrassingly small when compared with the experiments. When this constraint is relaxed the resistive instabilities persist, except possibly at zero or very small pressure. This seems to contradict the experimental observations that up to the point of the sawtooth crash the plasma is stable. We note, however, that the predicted resistive mode growth rates are very small indeed. It could be that the requirement of zero growth is too stringent or that the usual resistive MHD model is inadequate. This latter possibility was discussed in Section 4 for quite different reasons. There, the resistive MHD model was deemed optimistic, while here it seems overly pessimistic. 
[1] Soltwisch, H., Stodiek, W. Manickam, J., and Schluter, J., in Plasma Physics and Controlled Nuclear Fusion Research, Proc. 11th International Conference, Kyoto, 1986.

[2] West, W.P, Thomas, D.M., Grassie, J.S. and Zheng, S.B. Phys. Rev. Lett., 58 (1987), 2758

[3] O'Rourke, J., Blum, J. Cordey, J.G., Edwards, A., Gottardi, N., Keegan, B., Lazzaro, E., Magyar, G., Stephan, Y., Stubberfield, P., Veron, D. and Zasche, D., in Controlled Fusion and Plasma Heating, Proc. 15th European Conf., Dubrovnik, 1988, Vol12B, Part I, p155.

[4] Bussac, M.N., Pellat, R., Edery, D. and Soule, J.L, Phys. Rev. Letters, 35, (1975), 1638 
0

0

0

0

0

0

0

0

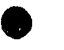

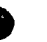




\section{ICRF SAWTOOTH STABILIZATION}

In this Section we describe a collaborative effort in which we participated in cooperation with the RF group at PPPL, in order to understand ICRF sawtooth stabilization in TFTR. Our primary role in this study was to evaluate the MHD stability of ICRF stabilized plasmas using data from the TRANSP analysis of these shots. These results were then used in a theoretical model based on energetic ion stabilization of $m=1$ kink instabilities to evaluate the stability of the plasmas. The main point of contact for this work was C. K. Phillips [1], who was responsible for integrating the MHD calculations with the energetic ion stabilization model and comparisons with experimental data. This Section summarizes the theoretical calculations with which we were directly involved. A short summary of the experimental observations of ICRF sawtooth stabilization is also given along with a comparison of the data to the theoretical calculations. Theory [4-7] suggests that the fast ions produced by the ICRF heating suppress sawteeth by stabilizing the $m=1$ instabilities which are believed to be responsible for the sawtooth oscillations. A qualitative correlation is observed between the experimental observations and model predictions, but further development of the theory coupled with more detailed experimental measurements is required in order to gain a quantitative understanding of the phenomenon.

The ICRF heating system on TFTR has been designed to provide up to $12.5 \mathrm{MW}$ of core plasma heating to complement the $35 \mathrm{MW}$ of neutral beam injection heating. These will be used to drive TFTR plasmas towards the $\mathrm{Q}=1$ breakeven point during D-T operations. To date, ICRF power levels up to $6 \mathrm{MW}$ at $47 \mathrm{MHz}$ have been coupled into the plasma in the out-of-phase launch mode with two out of the four antennas which comprise the completed system [2]. The stabilization of sawteeth has been observed both in RF only as well as in combined RF and NBI heated plasmas [1]. RF is also responsible for a reduction of core transport. It has also been demonstrated as efficient for heating the core in pellet-fueled plasmas [1]. Experiments are in progress to determine if it is possible to stabilize sawteeth in NBI-driven supershots by applying high power ICRF heating [1,2]. The mechanism is potentially important at high plasma currents when NBI heating alone is not sufficient to stabilize the sawtooth oscillations.

Stabilization of sawtooth oscillations using ICRF heating may be of particular importance during D-T operations on TFTR, if the accompanying increase in the energy confinement time and strong peaking of the core plasma temperatures leads to an enhancement of the fusion reactivity or $\beta_{\alpha}$. The ability to selectively heat core electrons or ions is a key property of ICRF heating that will be utilized to optimize fusion reactivity, 
QDT, and alpha particle beta, $\beta_{\alpha}$, in the high density, high temperature regimes expected during D-T operations on TFTR.

\subsection{Experimental ICRF Sawtooth Stabilization Observations}

Sawtooth Stabilization (SST) has been observed in both $\mathrm{D}$ and ${ }^{4} \mathrm{He}$ majority plasmas with minority $\mathrm{H}$ heating, as well as with ${ }^{3} \mathrm{He}$ minority heating in D NBI-produced plasmas. In $\mathrm{D}(\mathrm{H})$ plasmas, stabilization has been achieved for RF power levels as low as 2.2 MW with plasma currents ranging from 1.0 to $1.8 \mathrm{MA}$. For operation near the threshold power in $\mathrm{D}(\mathrm{H})$ plasmas, stabilization is observed only with on-axis heating. In addition, the threshold power in ${ }^{4} \mathrm{He}$ majority plasmas is dependent on plasma current. A higher threshold power for stabilization at the lower plasma current is consistent with theoretical models $[3,4,5,6]$, which suggest that the stabilizing influence of energetic trapped hot ions decreases if the hot ion pressure profile is not peaked and well-contained within the $\mathrm{q}=1$ volume. At $1.2 \mathrm{MA}$, approximately $75 \%$ of the RF power is deposited within the $\mathrm{q}=1$ volume, while at $1.4 \mathrm{MA}$, as much as $90 \%$ of the power is deposited within the $q=1$ volume.

The ICRF sawtooth stabilized phase in TFTR experiments has been observed to last up to many times the energy confinement time, as indicated in Fig. 8.1. The stabilized phase typically terminates with a very rapid growth of the $m=1$ island, leading into a large sawtooth crash. 


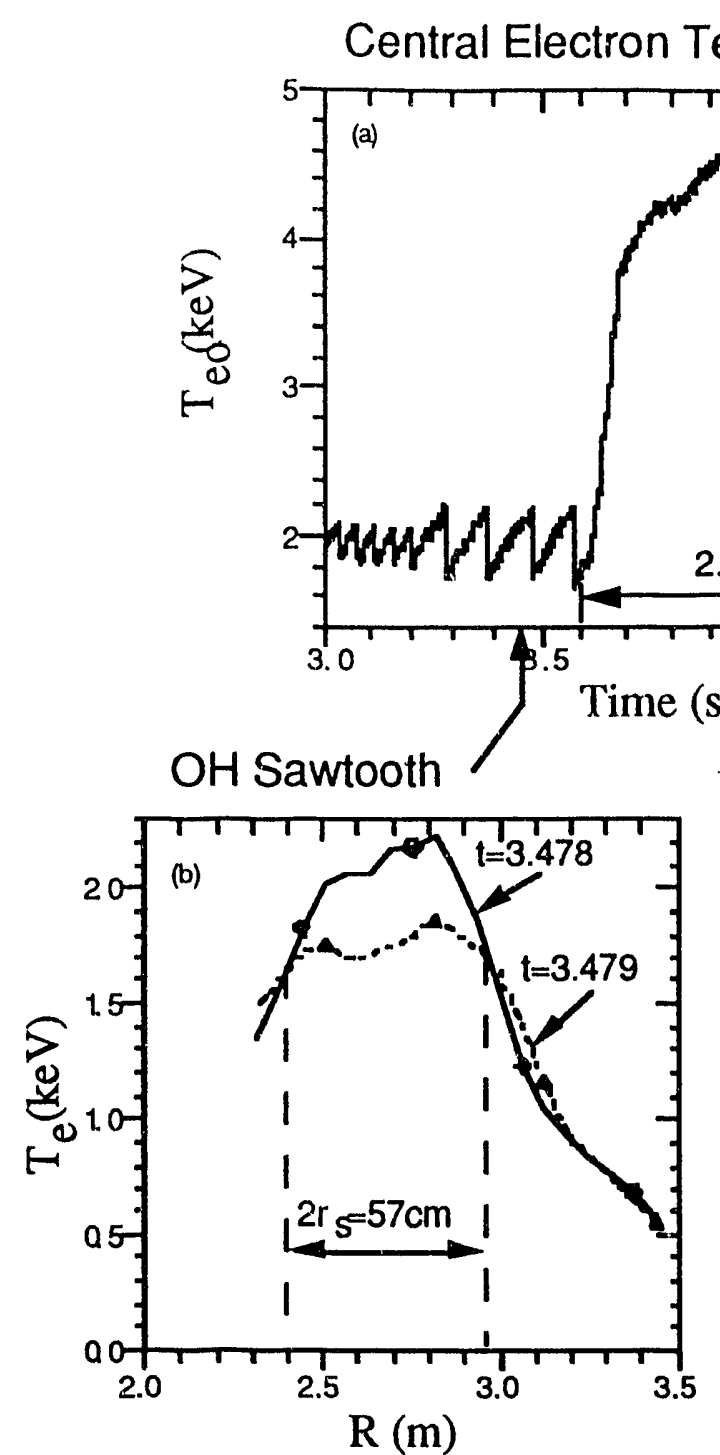

Fig. 8.1 The peaking of the temperature profile as well as the growth of the sawtooth inversion radius is shown. $\left[D(H) ; I_{p}=1.8 \mathrm{MA} ; n_{e}=2.5 \times 10^{13} \mathrm{~cm}^{-3}\right.$.

During the stabilized period, the electron temperature profile peaks significantly relative to the $\mathrm{OH}$ phase profile. The sawtooth inversion radius measured at the termination of the stabilized phase is also significantly larger than the corresponding radius during the $\mathrm{OH}$ phase, as shown in Fig. 8.1, implying that the current profile has also peaked up as a result of the stabilization. Preliminary measurements of the q profile, obtained using $\mathrm{Li}$ pellet injection[7], indicate that $\mathrm{q}(0)$ drops as low as 0.6 during the stabilized phase, consistent with the JET measurements[8] and with TRANSP calculations of the magnetic diffusion. 


\subsection{Theoretical Stability Calculations}

Theory suggests that the fast ions produced by the ICRF heating suppress sawteeth by stabilizing $\mathrm{m}=1$ internal kink instabilities believed to be responsible for sawtooth oscillations [3-6]. Assuming that the RF-driven energetic ions can be adequately described in terms of a tail distribution function, the dispersion relation governing $m=1$ ideal internal kink instabilities can be written as:

$$
\widehat{\gamma}_{1}+i[\Omega(\Omega-\Omega \cdot)]^{1 / 2}=\widehat{\beta}_{h}\left[\pi^{1 / 2}-2 \Lambda_{k}(0)\right]\left[\frac{1}{2}+\Omega+\Omega^{3 / 2} Z\left(\Omega^{1 / 2}\right)\right]
$$

where $\mathrm{Z}$ is the plasma dispersion function, $\Lambda_{\mathrm{k}}(0)$ represents the zero frequency response of the hot trapped ions $[3,5,6], \Omega=\omega / \omega_{d}, \omega_{d}=c T_{h} /\left(e B r_{1} R z_{h}\right)=$ the precession frequency of $a$ hot ion with effective temperature $T_{h}$ and charge $z_{h}, \Omega^{*}=\omega^{*}{ }_{i} / \omega_{d}$ where $\omega^{*}{ }_{i}$ is the ion diamagnetic frequency, $\widehat{\gamma}_{i}=\gamma_{\mathrm{I}} / \omega_{\mathrm{d}}, \beta_{\mathrm{h}}$ is the hot ion beta (defined below), $\hat{\beta}_{\mathrm{h}}=\beta_{\mathrm{h}} \omega_{\mathrm{A}} / \varepsilon$ $\omega_{\mathrm{d}}$, with $\varepsilon=r_{1} / R$, and $\omega_{\mathrm{A}}$ is the Alfvén frequency. Comparison of the experimental stabilized discharge parameters to the $\mathrm{m}=1$ ideal internal kink stability boundaries qualitatively supports the interpretation that the stabilization is due to the pressure of the hot ions. However, the experimental uncertainties and the theoretical simplifications can lead to changes of factors of 2 to 3 in the location of either the data points or the stability boundaries. A comparison of some TFTR data points to the predicted stabilization boundaries is displayed in Figs. 4.2 and 4.3. Since the boundaries depend sensitively on $\Lambda_{k}(0)$, a parameter which is not well determined $[3,5,6]$, the stable regions have been drawn for two representative values of $\Lambda_{k}(0)$. The ideal MHD growth rates for the data were evaluated with the ARES resistive MHD stability code. Experimental parameters are obtained directly from measurements or else are inferred from SNAP or TRANSP analyses. Estimates for $\beta_{h}$ have been evaluated in Fig. 4.2 using the integral formulation proposed by Coppi et al. [5]:

$$
\left\langle\beta_{\mathrm{h}}\right\rangle=\int_{0}^{1} \mathrm{~d} \tilde{\mathrm{r}} \tilde{\mathrm{r}}^{3 / 2} \frac{\mathrm{d}}{\mathrm{d} \tilde{\mathrm{r}}}\left[\tilde{\mathrm{r}}^{1 / 2} \mathrm{p}_{\perp \mathrm{h}}\right] \quad \text { where } \tilde{\mathrm{r}}=\frac{\mathrm{r}}{\mathrm{r}_{1}}
$$

and where $\mathrm{p}_{\perp h}$ is the perpendicular pressure of the energetic ions, 


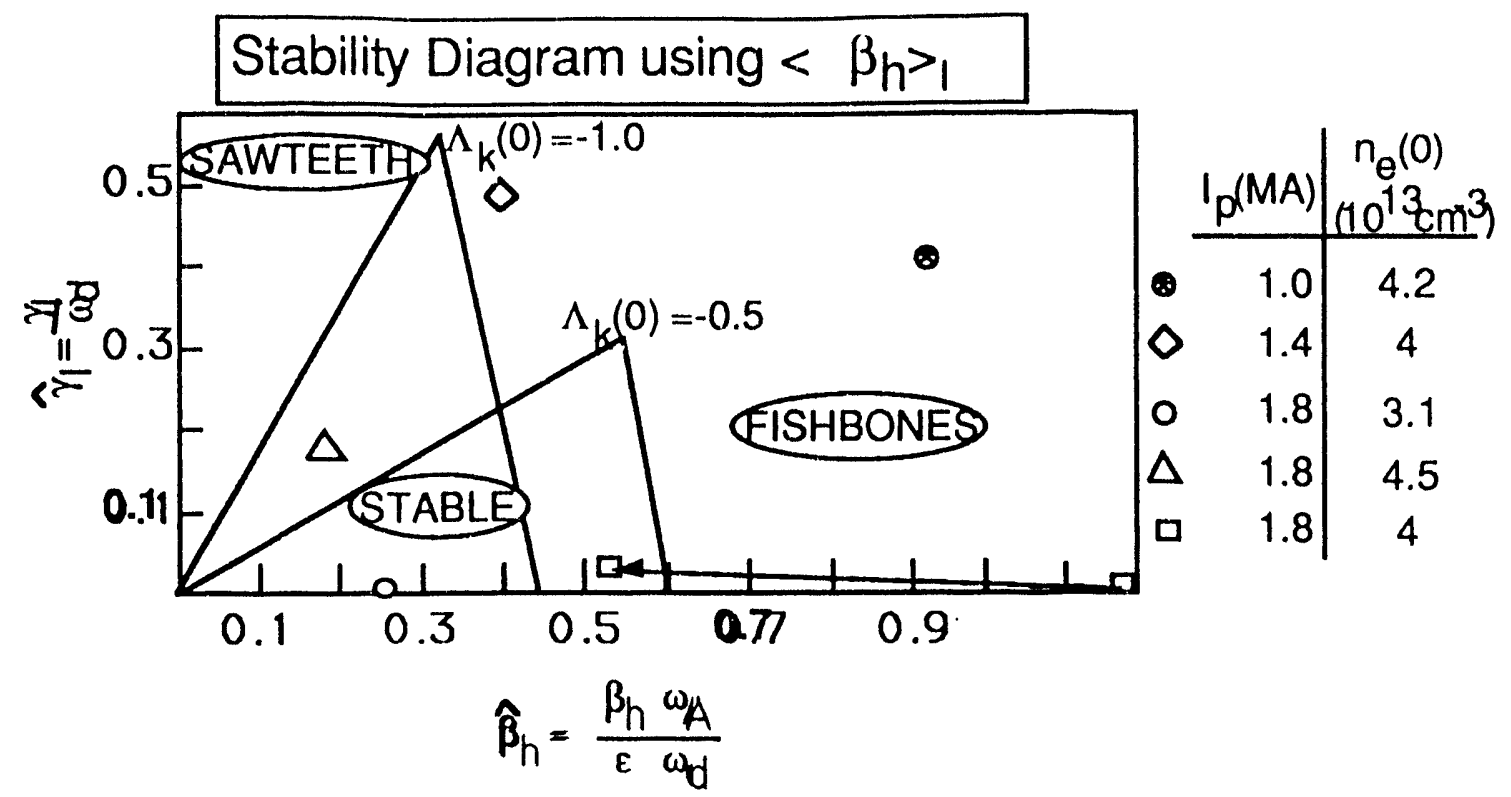

Fig. 8.2 TFTR data is plotted on the theoretical stability diagram using the integral form for $\beta \mathrm{h}$. The arrow indicates the direction of increasing time for a shot analyzed with TRANSP.

or else a volume estimate:

$$
\left\langle\beta_{\mathrm{h}}\right\rangle_{\mathrm{V}}=\left[\frac{8 \pi}{B_{1}^{2}}\right]\left[\frac{\mathrm{W}_{\perp h}}{\mathrm{~V}}\right]
$$

where $W_{\perp h}$ is the perpendicular stored energy in the hot ions, as used in Fig. 8.3. The time evolution for one specific discharge through stability phase space has been indicated in Figs. 8.2 and 8.3 with arrows. Throughout the SST phase, the discharge evolves towards the region of phase space characterized by sawtooth oscillations. 


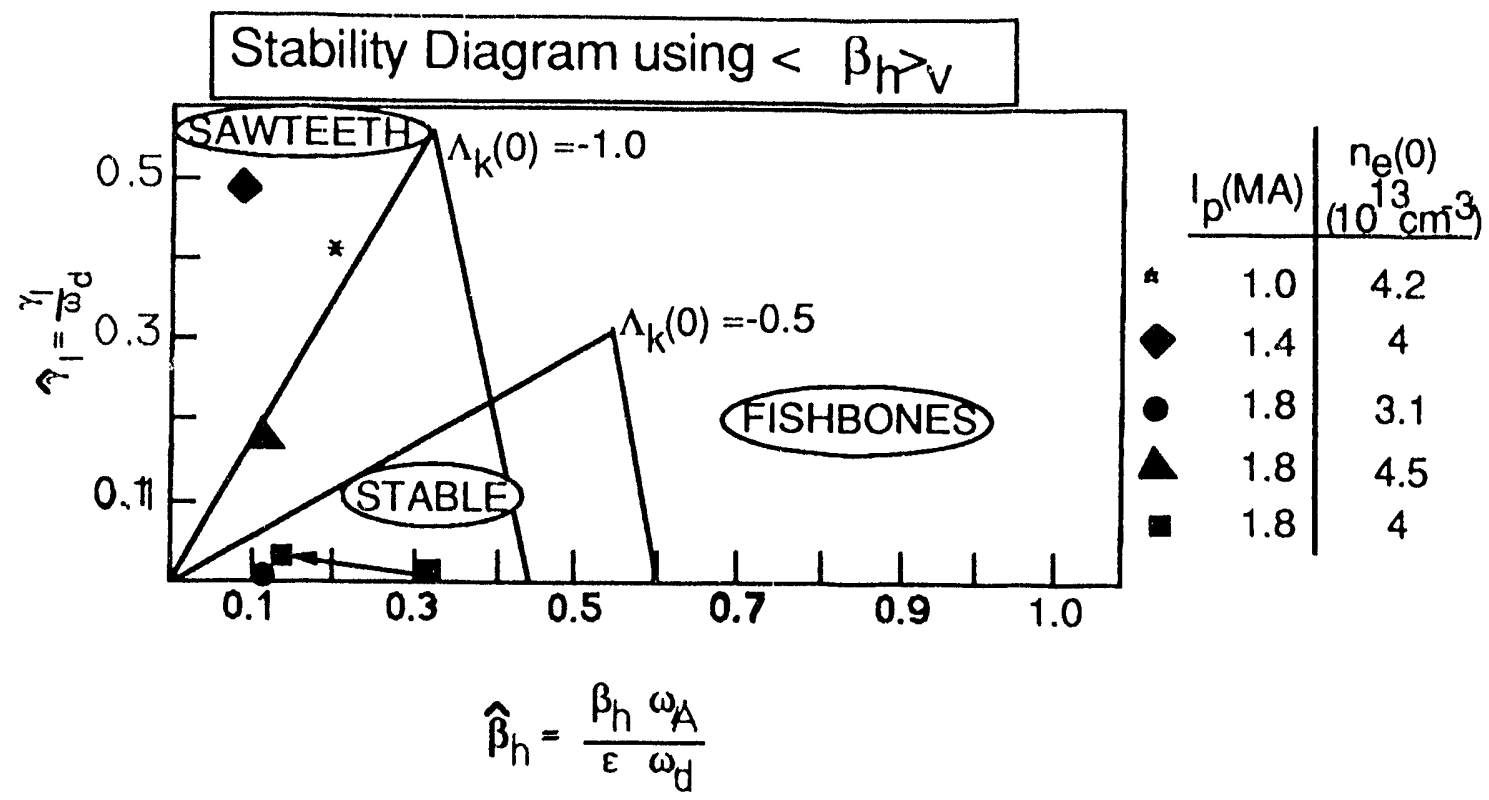

Fig. 8.3. TFIR data is plotted on the theoretical stability diagram using volume average for $\beta_{\mathrm{h}}$. The arrow indicates the direction of increasing time for a shot analyzed with TRANSP.

\subsection{Conclusions}

The MHD stability of ICRF stabilized plasmas was evaluated using ARES. These results were used in the evaluation of a theoretical model that includes stabilization of energetic ions. The results were compared with the experiments. A qualitative correlation has been found between the experimental observations of ICRF sawtooth stabilization and the theoretical predictions, given the large uncertainties in both the measurements and the theory. Further development of the theory coupled with more precise measurements should provide a more reliable basis for projections using the stabilization mechanism during D-T operations in TFTR. 
[1] C. K. Phillips, E. Fredrickson, G. Hammett, J. Hosea, K. McGuire, J. Stevens, J. R. Wilson, M. Bell, M. Bitter, R. oivin, K. Hill, H. Hsuan, D. Jassby, F. C. Jobes, D. McCune, D. K. Owens, H. Park, A. Ramsey, J. Schivvell, G. Schmidt, B. Stratton, E. Synakowski, G. Taylor, H. Towner, R. White, S. Zweben, M. W. Philiips, M. Hughes, E. Marmer, J. Snipes, J. Terry, Y. Nagayama, C. Bush, R. Godlfinger, D. Hoffman, D. N. Smithe, P. Colesock, 7th Topical Meeting on ICRF Heating, Aug 19-21 1991 Charlston, S.C.

[2] J. Hosea et. al., Plasma Phys. and Contr. Nucl. Fus. Res. 1990, Vol.1,IAEA, Vienna, 1991, pg.669.

[3] F.Porcelli, "Fast Particle Stabilisation", Invited Review Paper, 18th EPS Conf. on Contr. Fus. and Plasma Phys., Berlin 1991, to appear in Plasma Phys. and Contr. Fus.

[4] R.B. White et.al., Phys. Rev. Lett. 62, 539 (1989).

[5] B. Coppi et.al.., Phys. Rev. Lett. 63, 2733 (1989).

[6] C.Z. Cheng, Phys. Fluids B 2, 1427 (1990).

[7] E.S. Marmar and J.L. Terry, Rev. Sci. Inst. 61, 3081 (1990).

[8] D.J. Campbell et.al., Plasma Phys. and Contr. Nucl. Fus. Res. 1988, Vol.1, IAEA, Vienna,1989, pg. 377. 


\section{SUMMARY}

This report has described significant results in our ideal and resistive MHD stability studies which have evoked substantial interest among both experimentalists and theorists in the fusion community. Aspects of this work have been described at a number of meetings and conferences, a list of which is given in the Appendix. Part of this work is already published in the literature and the remainder is currently in preparation for publication prior to the end of this contract period. The conclusions from each of the main Sections of this report are summarized below.

Resistive MHD analysis of supershot data at relatively low $\beta(<0.4 \%)$ shows that the finite pressure stabilization of tearing modes predicted theoretically is very strong in these high $\beta_{p}$ plasmas. When this stabilizing influence is removed by arbitrarily introducing narrow flat-spots in the pressure profiles, there is some qualitative agreement between resistive MHD computation and the experiments. In particular, conditions are found where either the $2 / 1$ or $3 / 2$ tearing mode is excited with a region of overlap where both modes appear. The low $\beta$ supershots considered here lie in the region where the predicted instability is predominantly $3 / 2$ and near marginal for the $2 / 1$, as observed experimentally. When the computational predictions are compared with a large set of experimental data, however, there is no convincing correlation between tearing mode theory and experiment. Detailed analytic calculations conclude that, in the high temperature regimes that exist in TFTR for example, linear tearing mode theory omits important contributions from other hydrodynamic effects such as mass flow and viscosity.

Stability analysis of high $\beta$ supershots show that ballooning modes and infernal modes may explain the MHD activity observed during the experiments. Supershots with low central shear were found to be either very close, or in a regime unstable to infernal modes. The dominant mode depends on the trajectory the equilibrium follows in $\beta, q(0)$ space as time evolves. The $2 / 1$ mode requires $q(0)>1.5$ to occur, which may explain why this mode is not often observed. A likely scenario suggested by these stidies is that supershots can operate at or slightly in the ballooning mode threshold, with an increase in MHD activity but no collapse. However, if the current profile in the center becomes flat, a discrete infernal mode occurs leading to a beta collapse. Both infernal modes and ballooning mode instability regions in parameter space are surrounded by a narrow region unstable to resistive modes. No flattening of the pressure profile is required to excite these modes. However, in view of the narrowness of this region, it is likely these modes are simply resistively modified ballooning modes or infernal modes and not tearing modes. 
In a further study of the role of ideal, pressure driven modes, the numerical profile information obtained from TRANSP was approximated with analytic formulae to gauge the sensitivity of the stability results to the assumed equilibrium data. The limiting $\beta$ determined by the infernal modes mentioned above was shown to follow a Troyon-like scaling, $\beta_{\max }=\mathrm{C}_{\mathrm{T}} \mathrm{I} / \mathrm{aB}$, but with the coefficient $\mathrm{C}_{\mathrm{T}}$ sensitively dependent on the peakedness of the pressure profile. The maximum attainable $\beta$ can be severely limited when the pressure profile is highly peaked. These infernal modes are excited only when the shear within the rational surface of interest is small and, therefore, $q(0)$ is close to that rational value.

The $3 / 2$ infernal mode is the most unstable being most virulent when $q(0)=1.45$. Instabilities with larger toroidal mode numbers are also found at smaller values of $q(0)$. Interestingly, the $2 / 1$ mode is excited only when the pressure profile is very highly peaked. This, together with the requirement that $\mathrm{q}(0)$ must exceed $\sim 1.7$ before the instability is excited, could well be the reason that the $2 / 1$ mode is observed only very infrequently in supershot plasmas.

To quantify the role of the shear in exciting pressure driven modes, the shape of the q-profile was systematically altered. It was shown that, as the q-profile is steepened and the shear is correspondingly increased, the limiting $\beta$ for the infernal modes increases up to a point where small toroidal mode number ballooning modes appear. Further increasing the shear causes the limiting $\beta$ to decrease. These ballooning modes do not rely on elevated values of $q(0)$ and are excited over a wide range including $q(0)=1$.

Exploi atory studies of the role of ideal and resistive $1 / 1 \mathrm{MHD}$ modes show that the experimental pressure and current density profiles measured at the TEXTOR tokamak are near marginal stability to the ideal internal $1 / 1$ kink mode. In general, however, we were unable to find conditions stable to resistive modes (except in particular calculations which imposed the constraint of incompressible perturbations). Since this contradicts the experimental observations, our (preliminary) conclusion is that either the requirement of zero growth rate is too stringent or that the 'usual' resistive MHD model is inadequate.

The stability of ICRF driven plasmas was evaluated using ARES. These results were used in the evaluation of a theoretical model that includes stabilization of energetic ions. A qualitative correlation was found between the experimental observations of ICRF sawtooth stabilization and the theoretical predictions, given the large uncertainties in both the measurements and the theory. This suggests further work involving analysis of more 
experimental data and development of the theory with the goal of including more kinetic effects directly into the stability codes. This should provide a more reliable basis on which projections can be made for using the stabilization mechanism for D-T operations in TFTR. 
-

-

-

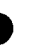

.

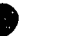

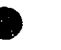


APPENDIX: Associated Publications and Presentations

(a) Journal Articles

1. 'Effect of Current Profile Shape on Second Region Access in High-Beta Tokamak Plasmas', M.W.Phillips, M.H.Hughes, A.M.M.Todd, M.Okabayashi, S.M.Kaye and B.LeBlanc, Phys. Fluids B, 2, (1990), 973

2. 'Edge Modeling of ICRF Heated Plasmas on PLT', I.S.Lehrman, P.L.Colestock and E.F.Jaeger, Nucl. Fusion, 30, (1990), 1399

3. '3-D MHD Studies of Sawtooth Oscillations and Pressure Driven Resistive Modes in Tokamaks', W. Park, D. A. Monticello, E. Fredrickson, B. Grek, K. McGuire, M. H. Hughes and M. W. Phillips, Plasma Physics and Controlled Nuclear Fusion Research 1990, Washington, D.C., Paper LAEA-CN-53/D-2-5.

4. 'Sawtooth Stabilization Studies on TFTR', C.K.Phillips, G.W.Hammet, J.Hosea, E.Marmar, M.W.Phillips, J.Snipes, J.Stevens, J.Terry, J.R.Wilson, M.Bell, M.Bitter, C.Bush, E.Fredrickson, K.Hill, D.Hoffman, H.Hsuan, M.Hughes, A.Janos, D.Jassby, K.McGuire, Y.Nagayama, D.K.Owens, H.Park, A.Ramsey, J.Schivell, E.Synakowski, B.Stratton, G.Taylor and TFTR Group, Proc. $18^{\text {th }}$ European Conf. on Controlled Fusion and Plasma Physics, Berlin, June 1991.

5. 'ICRF Heatimg on TFTR', C. K. Phillips, M.W.Phillips, M.H.Hughes et al., Radio Frequency Power in Plasmas, AIP Conference Proceedings 244, (ed. D. Batchelor) AIP, NY (1992), 88

6. 'Ion Cyclotron Range of Frequencies Stabilization of Sawteeth on the Tokamak Fusion Test Reactor', C.K.Phillips, J.Hosea, E.Marmar, M.W.Phillips, J.Snipes, J.Stevens, J.Terry, J.R.Wilson, M.Bell, M.Bitter, R.Boivin, C.Bush, C.Z.Cheng, D.Darrow, E.Fredrickson, R.Goldfinger, G.W.Hammet, K.Hill, D.Hoffman, W.Houlberh, H.Hsuan, M.Hughes, D.Jassby, D.McCune, K.McGuire, Y.Nagayama, D.K.Owens, H.Park, A.Ramsey, G.Schilling, J.Schivell, D.N.Smythe, B.Stratton, E.Synakowski, G.Taylor, H.Towner, R.White, S.Zweben and TFTR Group, Phys. Fluids B, 4, (1992), 2155

7. 'A Computer Model for Resistive MHD Analysis', M.H.Hughes, M.W.Phillips and R.G.Storer, to appear in Computer Physics Communications.

8. Theoretical Basis for Advanced Plasma Configurations', S.C.Jardin, A.Bhattacharjee, A.Bondeson, M.S.Chance, S.C.Cowley, G.Erikkson, J.M.Greene, M.Hughes, F.Hofman, R.Iacono, D.W.Ignat, N.Pomphrey, C.Kessel, H.Lutjens, J.Manickam, D.A.Monticello, F.W.Perkins, M.Phillips, A.H.Reiman, P.H.Rutherford, E.J.Valeo, L.Villard, D.J.Ward and C.Wang, Paper IAEA-CN-56/D-4-13-1, to appear in Plasma Physics and Controlled Nuclear Fusion Research, LAEA, Wurzburg, Germany, 1992.

9. 'A Model for Compressible, Resistive MHD Stability Analysis', M.H.Hughes and M.W.Phillips, in preparation, to be submitted for publication in Computer Physics Communications.

10. 'Pressure Driven Instabilities in TFTR, Supershot Plasmas', M.H.Hughes and M.W.Phillips, in preparation, to be submitted for publication in Nuclear Fusion 
11. 'Resistive Stability of Tearing Modes in Finite-Beta Plasmas', R.Iacono, A.Bhattacharjee, J.M.Greene, M.H.Hughes, in preparation, to submitted for publication in Phys. Fluids B

12. 'The Tokamak Sawtooth Process and Resistive MHD Stability Criteria', W.Stodiek and M.H.Hughes, in preparation, to be submitted to Phys. Rev. Letters.

(b) Reports

1. 'High Beta and Second Stability Region Transport and Stability Analysis : 1990 Technical Progress Report for DoE Grant \# DE FG02-89ER51124', M. H. Hughes, M. W. Phillips and A. M. M. Todd, Report No. DOE/ER51124-2, June 12 (1990).

2. 'High Beta and Second Stability Region Transport and Stability Analysis : 1991 Technical Progress Report for DoE Grant \# DE FG02-89ER51124', M. H. Hughes, M. W. Phillips and A. M. M. Todd, Report No. DOE/ER51124-4, September 5 (1991).

3. 'ICRF Stabilization of Sawteeth on TFTR', C.K.Phillips, J.Hosea, E.Marmar, M.W.Phillips, J.Snipes, J.Stevens, J.Terry, J.R.Wilson, M.Bell, M.Bitter, R.Boivin, C.Bush, C.Z.Cheng, D.Darrow, E.Fredrickson, R.Goldfinger, G.W.Hammet, K.Hill, D.Hoffman, W.Houlberh, H.Hsuan, M.Hughes, D.Jassby, D.McCune, K.McGuire, Y.Nagayama, D.K.Owens, H.Park, A.Ramsey, G.Schilling, J.Schivell, D.N.Smythe, B.Stratton, E.Synakowski, G.Taylor, H.Towner, R.White, S.Zweben and TFTR Group, Princeton University, Plasma Physics Report PPPL-2820, January (1992).

4. 'High Beta and Second Stability Region Transport and Stability Analysis : Final Report for DoE Grant \# DE FG02-89ER51124', M. H. Hughes, M. W. Phillips, A. M. M. Todd, J. Krishnaswami and R. Hartley, Report No. DOE/ER51124-7, September 18 (1992).

\section{(c) Conference Papers and Presentations}

1. 'Profile Control and Stable Access to the Second Region in PBX-M and TFTR', M.W.Phillips and M.H.Hughes, APS, Division of Plasma Physics Annual Meeting, Anaheim, CA, 1989.

2. 'Resistive MHD Stability Analysis of TFTR Supershots', M.H.Hughes and M.W.Phillips, Paper 3D22, Sherwood Fusion Theory Conference, Williamsburg, 1990.

3. 'Resistive Analysis of High-Beta MHD Activity in TFTR', M.W.Phillips, M.H.Hughes, K.M.McGuire, D.A.Monticello and W.Park, presented at Second TTF Transport Meeting, Hilton Head, February 1990. 
4. 'Resistive MHD Stability Properties of TFTR Supershots', M.H.Hughes and M.W.Phillips, Paper 1Q07, APS, Division of Plasma Physics Annual Meeting, Cincinnati, OH, 1990.

5. 'Resistive Stability Near the Transition to Second Region in TFTR', M.W.Phillips and M.H.Hughes, Paper 1Q11, APS, Division of Plasma Physics Annual Meeting, Cincinnati, OH, 1990.

6. 'Sawtooth Stabilization Studies on TFTR', C.K.Phillips, G.W.Hammett, J.C.Hosea, J.E.Stevens, J.R.Wilson, P.L.Colestock, D.Hoffman, M.W.Philips and M.H.Hughes, Paper 7P34, APS, Division of Plasma Physics Annual Meeting, Cincinnati, OH, 1990.

7. '3-D MHD Studies of Sawtooth Oscillations and Pressure Driven Resistive Modes in Tokamaks', W. Park, D. A. Monticello, E. Fredrickson, B. Grek, K. McGuire, M. H. Hughes and M. W. Phillips, Plasma Physics and Controlled Nuclear Fusion Research 1990, Washington, D.C., Paper IAEA-CN-53/D-2-5.

8. 'Resistive MHD Studies of TFTR Discharges', M.H.Hughes, M.W.Phillips, S.A.Sabbagh and R.V.Budny, Paper 2D06, Sherwood Fusion Theory Conference, Seattle,WA. 1991.

9. 'Ideal MHD Stability of High Poloidal Beta Equilibria in TFTR', S.A.Sabbagh, M.E.Mauel, G.A.Navratil, M.G.Bell, R.V.Budny, M.S.Chance, E.D.Fredrickson, S.C.Jardin, J.Manickam, D.C.McCune, K.M.McGuire, R.M.Wieland, M.C.Zarnstorff, M.W.Phillips, M.H.Hughes and J.Kesner, Paper 1C05, Sherwood Fusion Theory Conference, Seattle,WA. 1991.

10. 'Resistive Stability of High $\varepsilon \beta p$ TFTR Equilibria', M.H.Hughes and M.W.Phillips, presentation at US/USSR Exchange I8, Troitsk Institute, Moscow, September 15-18 1991.

11. 'Modeling of SRX Performance', M.H.Hughes, M.W.Phillips, S.A.Sabbagh and G.Navratil, presentation at US/USSR Exchange I8, Troitsk Institute, Moscow, September 15-18 1991.

12. 'Resistive MHD Stability Studies of Tokamak Discharges', M.H.Hughes and W.Stodiek, Paper 4Q14, APS, Division of Plasma Physics Annual Meeting, Tampa, FL, 1991.

13. The Tokamak Sawtooth and Disruption Processes', W.Stodiek and M.H.Hughes, Paper 4Q13, APS, Division of Plasma Physics Annual Meeting, Tampa, FL, 1991.

14. 'MHD Studies of High $\beta$ and Low $q$ Experiments in TFTR', M.W.Phillips, M.H.Hughes and C.K.Phillips,Paper 7S15, APS, Division of Plasma Physics Annual Meeting, Tampa, FL, 1991.

15. 'ICRF Stabilization of Sawteeth in TFTR', C.K.Phillips, M.H.Hughes and M.W.Phillips, APS, Division of Plasma Physics Annual Meeting, Tampa, FL, 1991. 
16. 'The Role of $(m, n)=(1,1)$ Mcdes in Disruptions on TFTR', E.Fredrickeson, K.M.McGuire, A.Janos, Y.Nagayama, M.Hughes, D.Monticello, W.Park, M.W.Phillips and J.Manickam, Paper 5E09, APS, Division of Plasma Physics Annual Meeting, Tampa, FL, 1991.

17. 'Resistive MHD Studies of High $\varepsilon \beta_{p}$ Plasmas', M.H.Hughes, presentation at HighBeta Workshop, General Atomics, San Diego, July 17, 1991.

18. 'Resistive Stability Near the Transition to the Second Region in TFTR', M.W.Phillips, presentation at High- Beta Workshop, General Atomic, San Diego, July 17th 1991.

19. 'Resistive MHD Studies of Supershot Plasmas', M.H.Hughes and M.W.Phillips, presentation to TFTR Physics Group Meeting, August $5^{\text {th }}, 1991$.

20. 'Sawtooth Stabilization Studies on TFTR', C.K.Phillips, G.W.Hammet, J.Hosea, E.Marmar, M.W.Phillips, J.Snipes, J.Stevens, J.Terry, J.R.Wilson, M.Bell, M.Bitter, C.Bush, E.Fredrickson, K.Hill, D.Hoffman, H.Hsuan, M.Hughes, A.Janos, D.Jassby, K.McGuire, Y.Nagayama, D.K.Owens, H.Park, A.Ramsey, J.Schivell, E.Synakowski, B.Stratton, G.Taylor and TFTR Group, Proc. $18^{\text {th }}$ European Conf. on Controlled Fusion and Plasma Physics, Berlin, June 1991

21. 'ICRF Heating on TFTR', C.K.Phillips, E.Fredrickson, G.W.Hammet, J.Hosea, K.McGuire, J.R.Wilson, M.Bell, M.Bitter, R.Boivin, K.Hill, H.Hsuan, D.Jassby, F.C.Jobes, D.McCune, D.K.Owens, H.Park, A.Ramsey, J.Schivell, G.Schmidt, B.Stratton, E.Synakowski, G.Taylor, H.Towner, R.White, S.Zweben, M.W.Phillips, M.Hughes, E.Marmar, J.Snipes, J.Terry, Y.Nagayama, C.Bush, R.Goldfinger, D.Hoffman, D.N.Smithe, Pcolestock and TFTR Group, Proc. AIP Conf. Radio Frequency Power in Plasmas, Charleston, S.C. 1991.

22. 'Resistive MHD Studies of High $\varepsilon \beta_{p}$ Experiments in TFTR', M.H.Hughes and M. W. Phillips, presentation at High Beta and Bootstrap Current Workshop, University of Wisconsin, December 11-13, 1991.

23. 'Current Profile Modification for Second Region Access', M. W. Phillips and M.H.Hughes, presentation at High Beta and Bootstrap Current Workshop, University of Wisconsin, December 11-13, 1991.

24. 'Stability Analysis of the Internal $1 / 1$ Mode in Tokamaks and Limitations of the Resistive MHD Model', M.H.Hughes, W.Stodiek, J.Manickam and J.L.Johnson, Paper 1C27, Sherwood Fusion Theory Conference, Santa Fe, NM, 1992.

25. 'Effect of Finite Pressure on Tearing Modes', A.Bhattacharjee, R.Iacono, C.Ronchi, J.M.Greene and M.H.Hughes, Paper 1C44, Sherwood Fusion Theory Conference, Santa Fe, NM, 1992.

26. Theoretical Basis for Advanced Plasma Configurations', S.C.Jardin, A.Bhattacharjee, A.Bondeson, M.S.Chance, S.C.Cowley, G.Erikkson, J.M.Greene, M.Hughes, F.Hofman, R.Iacono, D.W.Ignat, N.Pomphrey, C.Kessel, H.Lutjens, J.Manickam, D.A.Monticello, F.W.Perkins, M.Phillips, A.H.Reiman, P.H.Rutherford, E.J.Valeo, L.Villard, D.J.Ward and C.Wang, Paper IAEA-CN-56/D-4-13-1, to appear in Plasma Physics and Controlled Nuclear Fusion Research, IAEA, Wurzburg, Germany, 1992. 
27. 'Pressure Driven Instabilities in Supershot Plasmas', M.H.Hughes, M. W. Phillips and E. Fredrickson, to be presented at the DPP APS Meeting, November 1992, Seattle, WA.

28. 'Stability Analysis of MHD Activity in TFTR High Beta Supershot Plasmas', M.H.Hughes and M. W. Phillips, to be presented at the US-Japan Workshop on the Theory of High Beta Plasmas, November 11-13 1992, San Diego, CA. 

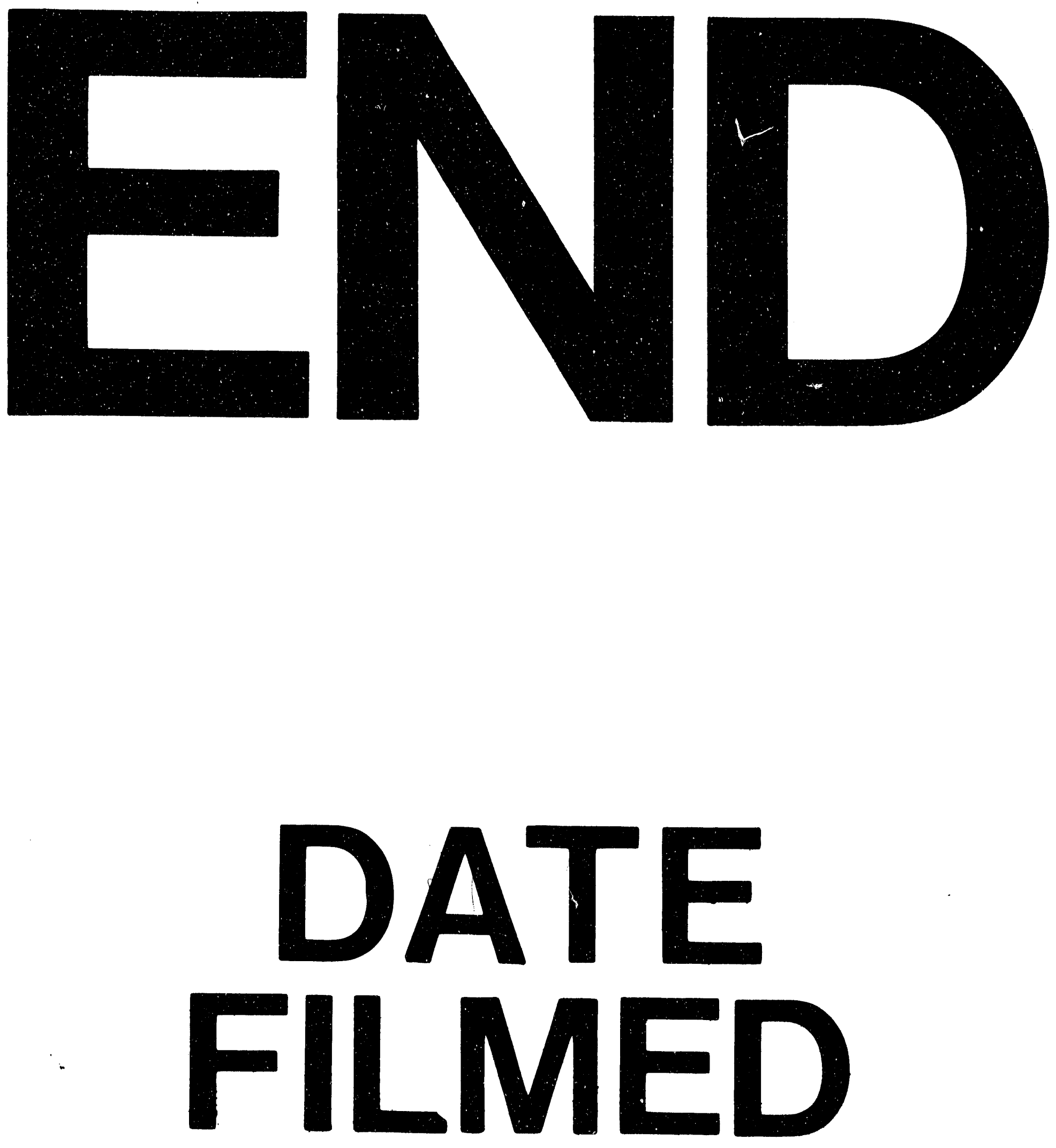

1

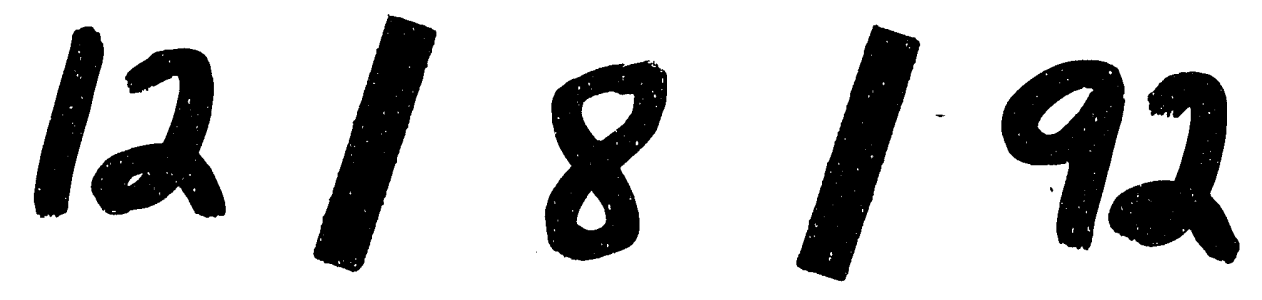


